\title{
SMART MATERIAL ELECTROHYDROSTATIC ACTUATOR FOR INTELLIGENT TRANSPORTATION SYSTEMS
}

\author{
A Thesis \\ Presented in Partial Fulfillment of the Requirements for \\ the Degree Master of Science in the \\ Graduate School of The Ohio State University \\ By
}

Michael J. Rupinsky, B.S.

$* * * * *$

The Ohio State University

2006

Master's Examination Committee:

Approved by

Dr. Marcelo Dapino, Adviser

Dr. Krishnaswamy (Cheena) Srinivasan

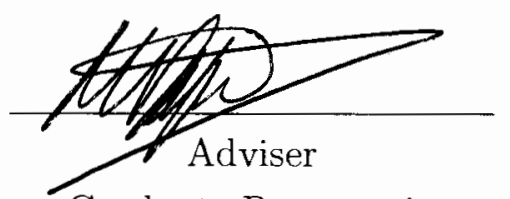

Graduate Program in Mechanical Engineering 
(C) Copyright by

Michael J. Rupinsky

2006 


\section{ABSTRACT}

Future intelligent transportation systems require actuation systems that are lightweight, compact and have a large power density. Due to their solid-state operation, fast frequency response, and high power-to-weight ratio, electrohydrostatic actuators (EHAs) based on smart materials are attractive as a replacement for conventional hydraulic actuators. Unlike conventional hydraulic systems, EHAs use smart material pumps which are driven electrically and integrated within the EHA rather than being mechanically connected to the vehicle's engine, thus precluding the need for accessory gear boxes and plumbing throughout the vehicle. This also permits the EHA to achieve much higher frequency responses, much lower overall power consumption, and increased reliability. The automotive and aerospace industries currently are the leading sectors in EHA research. Specific automotive uses include active suspension concepts, fly-by-wire steering and braking; aircraft uses include landing gear actuation and adaptive morphing of wings for advanced aerodynamic control.

A smart material deforms and changes its properties when exposed to external fields, for example electric fields (piezoelectric materials) or magnetic fields (magnetostrictive materials). Since smart materials produce small-stroke, large-force, highfrequency motion output in response to external fields, methods for transmitting small vibrations into large deformations and forces are needed. This document is focused on the development of a smart material-based pump for EHAs in which the mechanical 
vibrations produced by a magnetostrictive alloy, terbium-iron-dysprosium, are rectified by means of diode-type mechanical reed valves. A prototype pump is designed and constructed. Measurements conducted on this system demonstrate the ability of the reed valves to effectively rectify the oscillatory pressure pulses created by the magnetostrictive material and create unidirectional fluid flow. A maximum blocked pressure differential of $1100 \mathrm{psi}$ is achieved with a power consumption of $84 \mathrm{~W}$. A dynamic system model of the magnetostrictive pump with an attached hydraulic actuator is presented. The model is developed by using a lumped parameter analysis on the hydraulic circuit and using Newton's laws to couple the hydraulic and the mechanical domains. Coupling between mechanical and electrical domains is achieved by formulating electroacoustic transduction equations. Coupled differential equations of the system level model are written in state space form and solved numerically in MATLAB. The solver assumes a linear system for each time step and utilizes the values at previous times as initial conditions in order to march through time. The model can be easily modified in order to accurately model testing of the pump. The dynamic system model aids in validating the device operation and gives insight into future design modifications aimed at increasing the pressure output. Calculation of mechanical and electrical impedances allows analysis of the effect of hydraulic loading on pump operation. 


\section{ACKNOWLEDGMENTS}

The success of this research is attributed to the helpful assistance received from many individuals.

I thank the project adviser and Master's Examination Committee member, Prof. Marcelo Dapino, for his guidance, support and the opportunity to work in the Smart Materials and Structures Laboratory. I also thank Prof. Cheena Srinivasan, the second member on my Master's Examination Committee, for his inquisitive questions and comments about this research.

I'd like to thank my family and friends for their continued love and support throughout my college career. Thank you Mom, Dad, Chris, Jodie, Katie and Janelle for everything you've done for me. You motivate me to accomplish my dreams and goals.

I also would like to thank to my lab colleagues during my two year span in the Smart Materials and Structures Laboratory for their advice and friendship. In particular, thank you Dave Nosse for your help during the transition between projects.

I thank Gary Gardner and Keith Rogers in the Mechanical Engineering machine shop and Spellacy Machining and Fabricating for fulfilling several machining tasks, without whom component fabrication would have been tedious and cumbersome.

I am grateful to the Mechanical Engineering department for providing the instruction, facilities, and resources which assisted this research. I would also like to thank 
DANA Corporation, specifically Gerry McCann, for providing the funding for this research and Dr. Julie Slaughter for supplying the Terfenol-D sample. 


\section{VITA}

September 26, $1981 \ldots \ldots \ldots \ldots \ldots \ldots \ldots$. Born - Warren, Ohio, U.S.A.

May $2004 \ldots \ldots \ldots \ldots \ldots \ldots \ldots \ldots \ldots$. B.S. Engineering Physics Cum Laude, John Carroll University

2004-present .........................Graduate Research Associate, The Ohio State University

\section{FIELDS OF STUDY}

Major Field: Mechanical Engineering

Studies in Smart Materials and Structures: Prof. Marcelo Dapino

Major Field: Engineering Physics

Studies in Electrical Engineering: Prof. Klaus Fritsch 


\section{TABLE OF CONTENTS}

Page

Abstract ......................... ii

Acknowledgments ........................... iv

Vita ............................... vi

List of Tables ........................ . . .

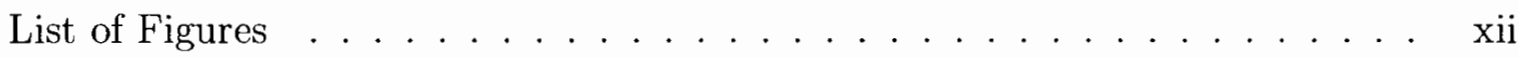

Chapters:

1. Introduction .......................... 1

2. Background Information . . . . . . . . . . . . 3

2.1 Motivation for Research . . . . . . . . . . . . . . . 3

2.2 Smart Materials . . . . . . . . . . . . . . . . 5

2.2.1 Piezoelectric Materials . . . . . . . . . . . . . . . 8

2.2 .2 Magnetostrictive Materials . . . . . . . . . . . . . 11

2.2.3 Smart Material Pumps . . . . . . . . . . . . . 20

3. Linear Dynamic System Model _... . . . . . . . . . . . . . . . 24

3.1 Mechanical Model of Terfenol-D Pump . . . . . . . . . . . . . 25

3.2 Fluid Subsystem . . . . . . . . . . . . . . . . . . . . . 28

3.3 State Space Modeling of Complete EHA . . . . . . . . . . . . . . . 31

3.4 Linear Magnetostrictive Modeling . . . . . . . . . . . . . . 35

3.5 Electroacoustics Theory . . . . . . . . . . . . . . . . . . . . 36

$3.5 .1 \quad$ Electrical Impedance Analysis . . . . . . . . . . . . . . 37 
3.5.2 Electroacoustics Equations . . . . . . . . . . . . 43

3.5.3 Modified Blocked Impedances . . . . . . . . . . . . . . . . . . 45

3.5 .4 Load Impedance . . . . . . . . . . . . . . . . . . 47

3.6 Full System Model and Simulations . . . . . . . . . . . . . . . 49

4. Terfenol-D Pump Design and Testing . . . . . . . . . . . . . . 53

4.1 Mechanical Design . . . . . . . . . . . . . . . . 53

4.1 .1 Reed Design . . . . . . . . . . . . . . . . . 58

4.1 .2 Magnetic Circuit . . . . . . . . . . . . . . . . . . 65

4.1 .3 Hydraulic Interface . . . . . . . . . . . . . . . 67

4.2 Experimental Setup . . . . . . . . . . . . . . . 70

4.2.1 Removal of Entrained Air . . . . . . . . . . . . 70

4.2 .2 Terfenol-D Preload . . . . . . . . . . . . . . . 72

4.2 .3 Bias Pressure . . . . . . . . . . . . . . . . . 74

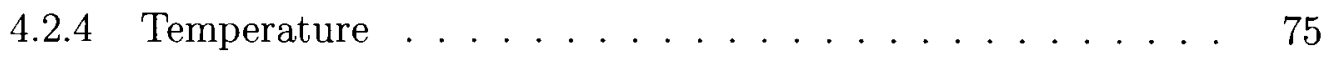

4.3 Data Collection . . . . . . . . . . . . . . . . . . . 75

4.4 Test Parameters . . . . . . . . . . . . . . . . . . . . . . 77

5. Results and Discussion . . . . . . . . . . . . . . . . 79

5.1 Dynamic Testing and Model Simulation . . . . . . . . . . 79

5.2 Harmonic Testing and Model Simulation . . . . . . . . . . . . . . 88

$5.2 .1 \quad$ Blocked Output Testing . . . . . . . . . . . . . . 88

5.2 .2 No-Load Testing . . . . . . . . . . . . . . . . . . 90

5.3 Results Summary . . . . . . . . . . . . . . . . . . . 93

6. Concluding Remarks . . . . . . . . . . . . . . . . . . . . . . . 98

6.1 Summary . . . . . . . . . . . . . . . . . . . . . . . . . . . 98

6.2 Future Work . . . . . . . . . . . . . . . . . . . . . 99

Appendices:

A. System Parameters for Dynamic System Model . . . . . . . . . . . . . 101

B. Terfenol-D Transducer Assembly and Part Drawings . . . . . . . . . 103

B.1 Magnet Alignment . . . . . . . . . . . . . . . . . . 103

B.2 CAD Drawings and Additional Pictures ........... 104 
C. Preliminary Magnetostrictive Motor and Pump . . . . . . . . . . . . 115

C.1 Terfenol-D Motor Unloaded Resonance . . . . . . . . . . . . . . . 115

C.2 Blocked Output Measurements . . . . . . . . . . . . . . . 117

C.3 Issues with Pump Design . . . . . . . . . . . . . . . . . . 122

D. Magnetorheological Valve Development . . . . . . . . . . . . . . . . . . 124

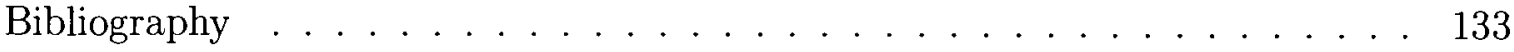




\section{LIST OF TABLES}

Table

Page

4.1 Material properties for materials being considered for the reed valves: type 301 full hardened stainless steel and 1095 blue tempered and polished spring steel. The material properties given are material density $\rho_{s}$, modulus of elasticity $E$, yield strength $S_{y}$ and ultimate tensile

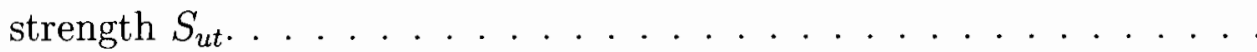

4.2 Natural frequencies of reed valve flap for thickness 0.005 in, 0.010 in and 0.015 in

4.3 Maximum deflection of the reed flap tip for thicknesses of 0.005 in, 0.010 in and 0.015 in. . . . . . . . . . . . . . . . .

4.4 Effect of various bias pressures on pump performance for a $1 \mathrm{~Hz} / 20 \mathrm{~A}$ (0 to peak) sinusoidal input. . . . . . . . . . . . . 74

4.5 Thermal coefficients of Etrema Terfenol-D. . . . . . . . . . . 75

5.1 Bandwidths of the various sensors used in testing. . . . . . . . .

5.2 Location of resonance, antiresonance and the respective magnetomechanical coupling coefficients for the experimental data and the dynamic system model calculated from equation $(3.27) \ldots \ldots \ldots$

5.3 Steady state pressure differential, leakage pressure during reed closure and power consumption resulting from harmonic testing of the EHA at a bias pressure of $250 \mathrm{psi}$ and for sinusoidal input current with an amplitude of $10 \mathrm{~A}$

5.4 No-load velocities resulting from harmonic testing of the EHA at a bias pressure of $250 \mathrm{psi}$ and input current with an amplitude of $5 \mathrm{~A}$. Reeds used are 0.005 in thick. . . . . . . . . . . . . . . 
A.1 Mechanical, electrical and fluid parameters associated with the magnetostrictive pump used in the dynamic system model. . . . . . . . . 101

A.2 Hydraulic fluid parameters for Mobil DTE 20 series hydraulic oil used in this research. . . . . . . . . . . . . . . . . . 102

D.1 Volume flow rates for various diameters of the drive piston driven at a resonance located at $2700 \mathrm{~Hz}$. The magnitudes of deflections of the Terfenol-D rod were calculated using equation (D.7) . . . . . . . 127

D.2 Magnetic characteristics of the three different MR valve geometries calculated from FEMM and retrieved from Figures D.1-D.2. . . . . . 129

D.3 Results from MRFV blocked pressure differentials of three different MR valve geometries. . . . . . . . . . . . . . . . . . . . . 129 


\section{LIST OF FIGURES}

Figure

2.1 Schematic of MR dampers for (a) small-scale/automotive and (b) large scale/seismic applications $[20] \ldots \ldots \ldots \ldots 7$

2.2 Schematic of SMA operation and material phases. The original, undeformed shape can be plastically deformed through mechanical forces. Application of heat returns the SMA to the memorized, undeformed shape. . . . . . . . . . . . . . . .

2.3 (a) Surface charge due to a spontaneous polarization, (b) twinned $180^{\circ}$ domains which form to minimize electrostatic energy and (c) twinned $90^{\circ}$ and $180^{\circ}$ domain structure for $\mathrm{BaTiO}_{3} \ldots \ldots \ldots 11$

2.4 (a) Tetragonal form of $\mathrm{PbTiO}_{3}$ and spontaneous polarization $P_{0}$, (b) indirect piezoelectric effect and direct piezoelectric effects (c) and (d).

2.5 Linear magnetostriction illustrated by the state of magnetic domains: (a) isotropic material in the disordered paramagnetic phase above $T_{c}$ modeled with spheres, (b) ellipsoids representing the order due to the spontaneous magnetization of the ferromagnetic phase once cooled through its Curie temperature with no applied field $H$, (c) reversible magnetic moment alignment with an applied magnetic field $H$, and (d) irreversible domain wall rotation from a saturating magnetic field $H$. The spontaneous and saturation magnetostriction $\lambda_{0}$ and $\lambda_{s}$ and total strain $e$ due to the orientation of domains in the field direction are noted. 15

2.6 (a) Relative magnetization $M / M_{s}$ and (b) magnetostriction $\epsilon$ produced by a sinusoidal magnetic field $H$ for Terfenol-D. . . . . . . . . . . 16

2.7 Crystallographic orientations in monolithic Terfenol-D. . . . . . . 17 
2.8 Magnetic domains in a Terfenol-D rod with crystallographic alignment (a); (b) Orientation of unstressed rod without an applied magnetic field. (c) Orientation of pre-stressed rod with no applied field and strain $\Delta \epsilon$ due to compression. (d) Orientation of pre-stressed rod when field is applied in the axial direction of the rod.

2.9 Magnetostrictive rotary transducers: (a) hybrid magnetostrictive/piezoelectric type $[25]$ and (b) elliptical type $[1] . \ldots \ldots \ldots \ldots$

2.10 Magnetostrictive sonar transducers: (a) Tonpilz [7], (b) flextensional, (c) square ring type and (d) hybrid magnetostrictive/piezoelectric type [5]. 19

2.11 Schematic of piezoelectric-hydraulic pump with active unimorph disk valves to control flow at inlet and outlet [22]. . . . . . . . . . 22

2.12 Solid model of a typical Terfenol-D transducer design. . . . . . . . . . 22

3.1 Schematic illustrating the fluid network of the Terfenol-D transducer while hydraulically attached to a piston. . . . . . . . . . . .

3.2 (a) Two degree of freedom vibratory model of the magnetostrictive pump and (b) orientation of Terfenol-D inside the transducer. . . . .

3.3 A cross-sectional view of the reed valve, illustrating its operation. (a) For a positive pressure gradient in the desired direction of fluid flow, the reed flap deflects and allows fluid flow. (b) For a positive pressure gradient in the opposite direction, the reed flap blocks the orifice and allows no flow. . . . . . . . . . . . . . . . . .

3.4 Symbolic diagrams of (a) a fluid capacitor and (b) a fluid resistor. [31] 31

3.5 Schematic representation of an electromechanical system. [17] . . . 36

3.6 Experimental total electrical impedance and inferred blocked electrical impedance for a Terfenol-D vibratory motor from Etrema Products, Inc. 38

3.7 Nyquist plot of the experimental total electrical impedance due to the motional component for a Terfenol-D vibratory motor from Etrema Products, Inc. . . . . . . . . . . . . . . . . . . 
3.8 Electrical admittance and inferred blocked electrical admittance for a Terfenol-D vibratory motor from Etrema Products, Inc. . . . . . . . . .

3.9 Nyquist plot of the experimental total electrical admittance for a Terfenol-

D vibratory motor from Etrema Products, Inc. . . . . . . . . . . .

3.10 Electrical drive-point impedance for both the Terfenol-D pump and dynamic system model. . . . . . . . . . . . . . . . 46

3.11 Circuit diagram showing the coil resistance and inductance in parallel to the capacitance due to the coil windings.

3.12 Model simulation of the blocked electrical impedance a low little damping value of $0.013 \ldots \ldots \ldots \ldots \ldots \ldots$

3.13 Model simulation and experimental test data: (a) Magnitude response of $Z_{e e}$, (b) Phase response of $Z_{e e}$, (c) Zoomed magnitude response of $Z_{e e}$ and (c) Maximum pressure time trace under blocked output conditions $(10 \mathrm{~Hz})$

4.1 Exploded solid model of the Terfenol-D pump. . . . . . . . . . . . . . 54

4.2 Exploded solid model of the Terfenol-D pump head subassembly. . . .

4.3 Compression tests for (a) Parker \#2-228 O-ring and (b) 0.005 in diaphragm.

4.4 (a) Picture of reed valve used in Terfenol-D transducer and (b) planar dimensions of reed valve. . . . . . . . . . . . . . . . . . . .

4.5 Picture of the Terfenol-D pump. . . . . . . . . . . . .

4.6 Pictures of the reed seats in the pump head (a) chamber housing and (b) port housing, (c) piston subassembly including the chamber and piston guide rings and (d) piston and diaphragm subassembly. . . .

4.7 FEMLAB model reed flap. . . . . . . . . . . . . . . . . 63

4.8 Strain vs. magnetic field (butterfly curve) for the Terfenol-D transducer illustrating the magnetic bias delivered by the Alnico $\mathrm{V}$ magnet stack. . . . . . . . . . . . . . . . . . . 
4.9 Magnetic induction along the length of the Terfenol-D rod, computed by FEMM for the magnetic circuit of the transducer. . . . . . . . 68

4.10 FEMM v 3.4 calculation of the magnetic flux lines and density of the magnetic induction in the magnetic circuit of the Terfenol-D pump. .

4.11 Schematic illustrating the setup for removal of air bubbles and entrained air.

4.12 Characteristic strain versus magnetic field curves for various prestresses of Terfenol-D . . . . . . . . . . . . . . . . . . . .

5.1 Results of stepped-sine testing of the Terfenol-D transducer: (a) Magnitude and (b) phase response of the electrical impedance frequency response function. . . . . . . . . . . . . . . . . . . . . . . 82

5.2 Results of dynamic testing of the Terfenol-D transducer: (a) Magnitude response of the acceleration/input current frequency response function and (b) magnitude response of the pressure/input current frequency response function. . . . . . . . . . . . . . . . . .

5.3 Magnitude response of the input current frequency response function.

5.4 Magnitude of the electrical drive-point impedance for experimental data and the model. The model predicts a resonance of $6250 \mathrm{~Hz}$ while resoance of the Terfenol-D transducer is at $5100 \mathrm{~Hz}$.

5.5 Comparison of model simulation and dynamic testing of the TerfenolD transducer: (a) Magnitude and (b) phase response of the electrical impedance $Z_{e e}$.

5.6 Comparison of model simulation and dynamic testing of the Terfenol-D transducer: (a) Zoomed magnitude response of $Z_{e e}$ and (b) magnitude response of the individual model components of $Z_{e e}, Z_{e}$ and $Z_{\text {mot }}$. . .

5.7 Nyquist plot of the electrical drive-point impedance for both experimental data and the dynamic system model. 
5.8 Harmonic blocked pressure testing alongside dynamic pressure data. Harmonic testing locates the frequency limitation of the reed valves at $1000 \mathrm{~Hz}$.

5.9 Pressure trace in the time domain for model simulation and harmonic test for a $10 \mathrm{~Hz} / 10$ A sinusoidal input. . . . . . . . . . . 97

B.1 Pump, total assembly except base. . . . . . . . . . . . . 104

B.2 Pump, base subassembly. . . . . . . . . . . . . 105

B.3 Pump, base. . . . . . . . . . . . . . . . 105

B.4 Pump, adjustor cap. . . . . . . . . . . . . 106

B.5 Pump, Terfenol-D rod seat. . . . . . . . . . 106

B.6 Pump, magnet assembly. . . . . . . . . . . . . . . . 107

B.7 Pump, head assembly. . . . . . . . . . . . . . . . 107

B.8 Pump, chamber housing. . . . . . . . . . . . . . . . . 108

B.9 Pump, port housing. . . . . . . . . . . . . . . . 108

B.10 Pump, piston subassembly. . . . . . . . . . . . . . . . . 109

B.11 Pump, piston in contact with Terfenol-D. . . . . . . . . . . 109

B.12 Pump, top part of piston in contact with fluid. . . . . . . . 110

B.13 Pump, diaphragm. Two were made. Thicknesses of 0.050 in and 0.005 in. 111

B.14 Pump, guide ring. . . . . . . . . . . . . . . . . . . 112

B.15 Pump, chamber ring. . . . . . . . . . . . . . . . . . 112

B.16 Pump, strut. . . . . . . . . . . . . . . . . . . 113

B.17 Pump pictures: (a) pump head chamber housing and (b) ports in the port housing. . . . . . . . . . . . . . 113 
B.18 Pictures of (a) the base subassembly and (d) one of the seven struts.

C.1 Frequency (a) and Phase (b) response for impedance transfer function, the acceleration/input current of the piston (c) and the magnitude of the input current spectral density $(\mathrm{d}) \ldots \ldots \ldots \ldots . \ldots 116$

C.2 Frequency response for acceleration/input current of the piston (a) and (c) and chamber pressure/input current (b) and (d). . . . . . . . 118

C.3 Experimental diagram for the blocked chamber measurements. The front chamber is filled with hydraulic fluid and blocked, while the back chamber is left open. A pressure sensor measures the pressure created by the pump.

C.4 Magnitude response for acceleration/input current of the piston (a), chamber pressure/input current (b) and magnitude and phase response of the impedance transfer function $(\mathrm{c})$ and $(\mathrm{d}) \ldots \ldots \ldots \ldots$

D.1 Magnetic induction versus magnetic field for MRF-132AD by Lord Corporation. . . . . . . . . . . . . . . . . 128

D.2 Yield stress versus magnetic induction for MRF-132AD by Lord Corporation. . . . . . . . . . . . . . . . . . 128

D.3 FEMM - MRFV \#1, existing design. . . . . . . . . . . . 130

D.4 FEMM - MRFV \#2, smaller existing design. . . . . . . . . . . 131

D.5 FEMM - MRFV \#3, proposed design. . . . . . . . . . . . 132 


\section{CHAPTER 1}

\section{INTRODUCTION}

Recent years have seen a significant increase in the use of smart material actuators in a variety of applications. A smart material deforms and changes its properties when exposed to external fields, for example electric fields (piezoelectric materials) or magnetic fields (magnetostrictive materials). Many applications have seen advances in compactness, response, control and reliability due to the implementation of smart materials.

This thesis presents the development and modeling of a magnetostrictive pump for Electrohydrostatic Actuators (EHAs) in which the magnetostrictive vibrations produced by a Terfenol-D rod are amplified to produce large deformations and forces. Integration of the magnetostrictive vibrations of Terfenol-D with hydraulic oil and fluid rectification techniques yields a fly-by-wire (power delivered through electrical connections), high pressure, unidirectional fluid pump. A linear model is developed coupling the electrical, mechanical and fluid regimes of the EHA.

Background information on the motivation, smart material properties and applications is outlined in Chapter 2. Greater detail is given on piezoelectric and magnetostrictive materials and is presented in Sections 2.2.1-2.2.2. Smart material pumps utilizing piezoelectric and magnetostrictive materials is presented in Section 2.2.3. 
The emphasis of the background information is placed on the magnetostrictive material Terfenol-D and smart material pumps.

The dynamic system model and magnetostrictive pump were developed concurrently but are separated into different chapters here for clarity. Chapter 3 describes the linear dynamic system model of the EHA. A summary is given on the methodology used for model development. The model has two parts; a state space model describing the coupling between the electrical, mechanical and fluid regimes for harmonic inputs and an electroacoustics model describing the dynamic effects of the pump over a specified frequency range. The model serves as a characteristic tool for the pump, correlates with experimental data and aids in future design considerations. Chapter 4 presents the design leading into the development of a Terfenol-D pump that produces a maximum pressure differential of 1100 psi. Details on the mechanical design, magnetic circuit, hydraulic interface and test parameters of the pump are given. Analysis of the results from model simulations and magnetostrictive pump testing is presented in Chapter 5 while concluding remarks on the project along with recommendations for future work are given in Chapter 6. The Appendix includes prior research that details the design and testing of a preliminary Terfenol-D pump consisting of a Terfenol-D vibratory motor from Etrema Products, Inc. coupled to a separate pump head capable of producing 100 psi pressure differential and also the design of a magnetorheological fluid valve. The knowledge gained from the preliminary pump led to the development of the pump outlined in Chapter 4 . 


\section{CHAPTER 2}

\section{BACKGROUND INFORMATION}

\subsection{Motivation for Research}

The automotive and aerospace industries can strongly benefit from research on compact fly-by-wire (power delivered through electrical connections) actuators to replace hydraulic or pneumatic systems with the goal of improving fuel economy, dynamic handling and packaging. These conventional actuation systems are bulky and are not ideal for future intelligent transportation systems based on high power density, miniaturized actuators. Fly-by-wire braking and steering, load leveling, suspension, controlled-banking, crash-worthiness, and noise and vibration exemplify some of the prospective automotive applications while landing gear actuation and flap actuators are some of the aerospace concepts under consideration. These applications have responses that depending on the regime of operation can range from slow to very fast, thus motivating the development of actuators capable of producing large work output concurrently with broadband response. Electrohydrostatic actuators (EHAs) utilizing smart materials are considered to satisfy the bandwidth requirement for structural motion and force control. EHAs are a class of smart material actuators that utilize hydraulic advantage along with a means for rectification of the smart 
material vibrations for linear actuation of external loads. EHAs prove advantageous over conventional hydraulic systems by eliminating the interface with the engine and the required external pumps and fluid lines. Smart materials allow for more compact packaging and thus a higher power to weight ratio than conventional actuation systems. The solid state nature of smart materials allows for the construction of actuators with few moving parts and enhanced reliability.

For optimization of the smart material pump, an accurate model is needed. A magnetostrictive material is nonlinear and hysteretic in nature but can be modeled linearly assuming that the material is working in its quasilinear range. From this assumption, a system of equations that couples the electrical, mechanical and fluid domains can be used to model the overall system response. Oates and Lynch (2001) utilize state space modeling techniques on a piezoelectric hydraulic pump and use this model as a design tool for future pump iterations [28]. The differential equations of each subsystem are coupled and solved simultaneously to obtain the overall system response. Sirohi et al. (2005) use transfer matrix analysis to model the dynamics of a piezo-hydraulic actuator, specifically the effects of viscosity and fluid transmission lines [19]. This analysis shows that the effect of increasing the tube length reduces the resonant frequency of the system and fluid viscosity affects the system in a manner similar to viscous damping. Downey and Dapino (2005) present a hybrid TerfenolD/PMN-PT transducer and model that provides accurate simulations of electrical and mechanical transducer behavior and also a framework for material property characterization [13]. The modeling of the Terfenol-D pump being investigated utilizes a lumped parameter analysis and Newton's laws to couple the mechanical and fluid 
domains, electroacoustic transduction equations for the coupling between mechanical and electrical domains and the constitutive relations of Terfenol-D relating the material properties with the electromechanical transduction coefficients.

A magnetostrictive pump for an EHA is presented in which the magnetostrictive vibrations are amplified to produce large deformations and forces. The motion amplification of the magnetostrictive vibrations is achieved by utilizing hydraulic advantage to convert magnetostrain into large forces and mechanical reed valves to rectify the pressure pulses from these vibrations, creating a high pressure unidirectional flow. The dynamic model is a tool that will aid in optimizing the magnetostrictive pump and allow for quick predictions of the device operation. This transducer has a performance target of moving a $4000 \mathrm{lb}$ payload at a velocity of $0.3 \mathrm{in} / \mathrm{s}$.

\section{$2.2 \quad$ Smart Materials}

A smart material is functionally defined as an engineered substance that transforms one form of input energy (i.e. electrical, magnetic, optical, thermal, etc.) into a different form of output energy. Smart materials are often labeled as controllable, intelligent, metamorphic, adaptive, active and compliant [9]. In this definition these materials are controllable in their behavior and properties and instinctive and reactive in that it responds to an external stimulus, as opposed to inert materials, but fails to meet criteria for "intelligence." The various scales of smart materials range from atomic to macroscopic. A more holistic definition of a smart material is one with intelligence and life features integrated in or around it with the goal to meet 
stated objectives and to provide adaptive functionality. A wide range of engineering applications utilize smart materials in a variety of actuation, sensor and control applications.

Piezoelectric materials convert energy between the electrical and mechanical regimes while magnetostrictive materials convert energy between the magnetic and mechanical regimes.

Magnetorheological (MR) fluids are suspensions of micron-sized magnetic particles, typically spherical iron particles, in a carrier fluid. The magnetorheological response of these fluids originates in the magnetization of the suspended particles in response to a magnetic field $H[20,29]$. The close range interaction among neighboring particles causes the particles to form chain-like columnar structures, called fibrils or tendrils, in the direction of the magnetic field. This leads to an increase in the apparent viscosity of the suspension as the flow becomes restricted in directions perpendicular to the fibrils. The mechanical energy needed to break the fibrils increases as the magnetic field is increased. In agreement with the magnetic nature of the particles, the dependence of yield stress with field exhibits saturation and a slight amount of hysteresis. MR fluids have been used in many commercial applications, especially as semiactive dampers. These semiactive dampers have seen use as automotive suspensions in select General Motors vehicles. Large scale MR fluid dampers have been used in vibration control in civil engineering applications to better protect against earthquakes and strong winds, see Figure 2.1.

Shape memory alloys (SMAs) are a class of smart materials which show a transition from a high symmetry austenite phase to a lower symmetry martensite phase. 


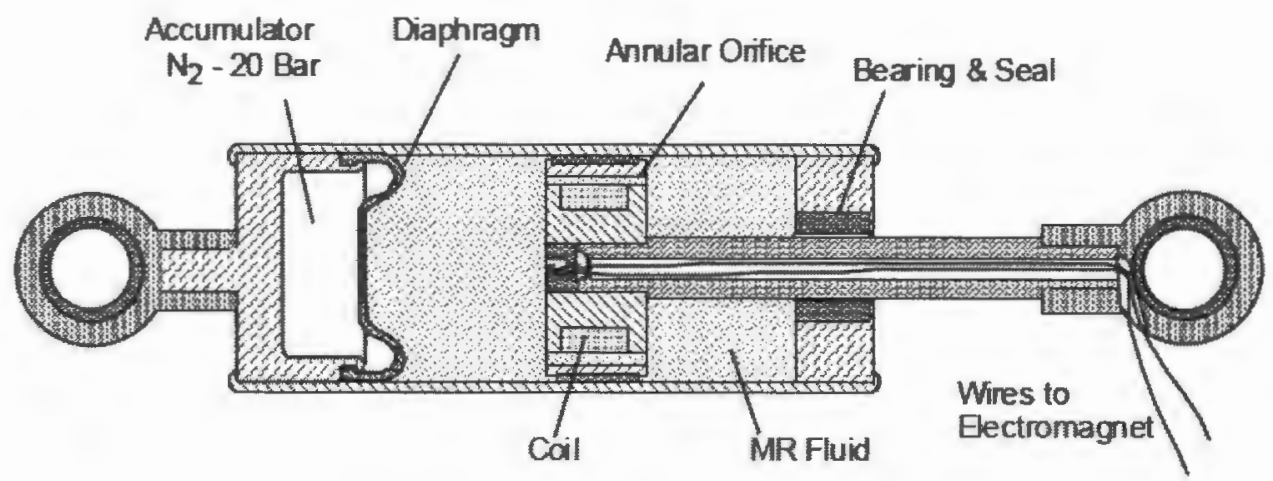

(a)

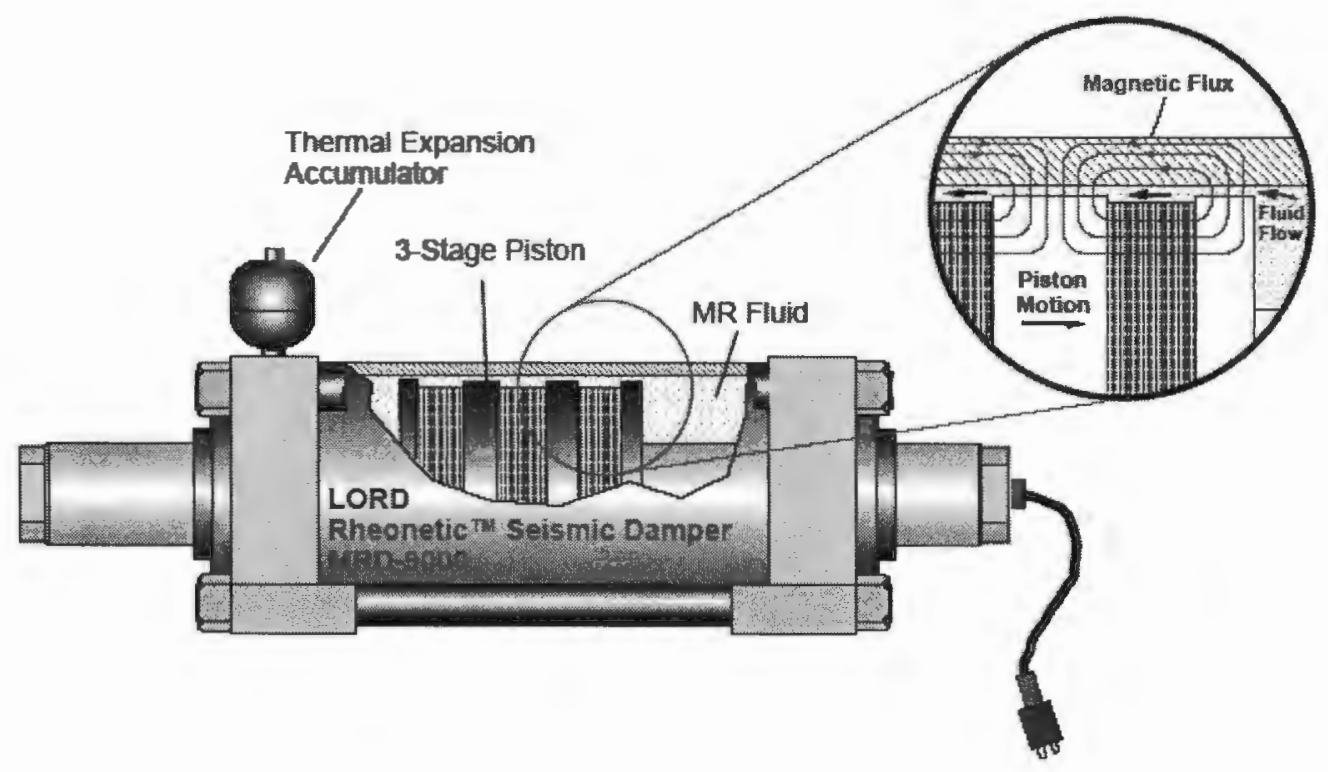

(b)

Figure 2.1: Schematic of MR dampers for (a) small-scale/automotive and (b) large scale/seismic applications [20].

SMAs can undergo significant plastic deformations in the low-temperature martensitic phase, and return to the original undeformed shape when transformed by heating to the high-temperature austenite phase. This behavior, known as the "shape memory effect," is illustrated in Figure 2.2. The volume fraction of the two phases in a 
shape memory material depends on temperature and stress. For elevated temperatures above the austenite transformation temperature, the SMA material has a cubic structure referred to as austenite. As the material is cooled below the martensitic transformation temperature, atoms within the material tilt in alternating directions. This low-temperature martensitic phase maintains the same overall shape as the hightemperature austenite phase. The application of a stress at low temperature plastically deforms the material through realignment of the internal martensite structure. To regain the original shape of the austenitic material, heat should be added to the material. The shape memory effect can also produce a tensile recovery force from a pre-strained SMA $[15,9]$. In other words, a deformed SMA attached to two points of a structure will contract and pull from the two points upon application of heat. Examples of these materials include copper-zinc-aluminum, copper-aluminum-nickel, and nickel-titanium (or Nitinol). A current application challenge of SMA's is their low bandwidth due to the time needed to thermally cycle between the low and high temperature phases. Applications in which this limitation has been circumvented include jet noise reduction, linear positioners, valves, switches, fire extinguishing equipment, tunable vibration absorbers, intravenous stents, orthodontic braces, and flexible eyeglasses.

\subsubsection{Piezoelectric Materials}

A piezoelectric material is one that is ferroelectric below the Curie temperature and exhibits an induced change in length when polarized. The term piezoelectricity refers to the generation of mechanical strain in response to applied electric fields (inverse effect) and conversely, the generation of electric charge due to applied stresses 


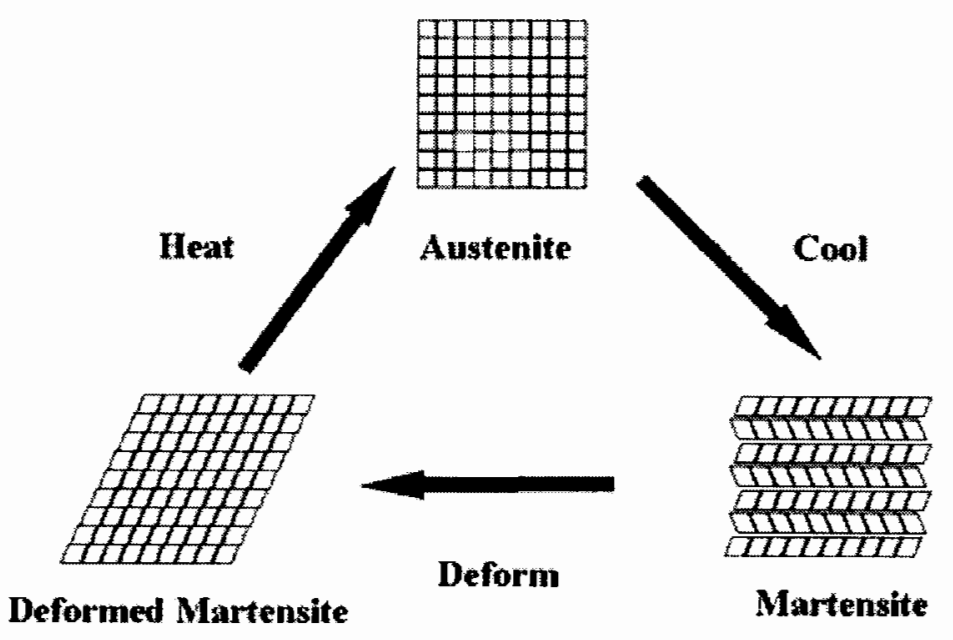

Figure 2.2: Schematic of SMA operation and material phases. The original, undeformed shape can be plastically deformed through mechanical forces. Application of heat returns the SMA to the memorized, undeformed shape.

(direct effect). The direct piezoelectric effect was first observed by Pierre and Jacque Curie in 1880 in tourmaline but was later extended to quartz, sugar cane and Rochelle salt. The dual, actuator/sensor capabilities were first illustrated by Paul Langevin in 1916 when he sandwiched quartz crystal between two plates and used it to send and receive sound waves in water. This transducer was a precursor to modern sonar devices which often use smart materials. Barium titanate $\left(\mathrm{BaTiO}_{3}\right)$, lead zirconate titanate (PZT) and lead magnesium niobate (PMN) are examples of modern piezoceramic materials.

Piezoelectric materials exhibit spontaneous polarization and a domain structure when in the ferroelectric phase. As the material is cooled below the Curie temperature, surface charge develops on the piezoelectric material from the electric field $E_{0}$ due to the spontaneous polarization $P_{0}$, as illustrated in Figure 2.3(a). In order to 
minimize the electrostatic energy, dipoles align themselves in twinned $180^{\circ}$ domains. A second mechanism which occurs as the material is cooled below the Curie temperature is the generation of thermal stresses [33]. Stresses in the direction of $E_{0}$ will not effect the $180^{\circ}$ domain structures but instead lead to the formation of $90^{\circ}$ domain walls, as in Figure 2.3(c), which occure to minimize the ferroelastic energy. The Tetragonal form of lead titanate $\left(\mathrm{PbTiO}_{3}\right)$ with a spontaneous polarization, along with the indirect and direct piezoelectric effects are illustrated in Figure 2.4. The application of an electric field $E$ induces a strain $\epsilon$, Figure 2.4(b). Changes in the polarization in lead titanate due to application of low tension stresses $\sigma$ are shown in Figure 2.4(c) and (d). It is noted that both the indirect and direct piezoelectric effects refer to linear and reversible electromechanical mechanisms which occur well before ferroelectric or ferroelastic switching [33].

Piezoelectric materials are attractive for smart material transducers due to their lightweight and compact nature, low cost and moderately linear strain vs. electric field response at lower fields. The linear strain at lower electric fields is modeled by the linear constitutive piezoelectric equations relating polarization $P$ and strain $\epsilon$ with stress $\sigma$ and electric field $E$,

$$
\begin{aligned}
P & =d \sigma+\chi_{\sigma} E \\
\epsilon & =s^{E} \sigma+d^{*} E
\end{aligned}
$$

where $d=d^{*}$ is the piezoelectric coupling coefficient, $\chi_{\sigma}$ is the dielectric susceptibility measured at constant stress and $s^{E}$ is the compliance measured at constant field. Some applications of piezoelectric materials include inchworm actuators, gyroscopes, accelerometers, micro-positioning devices, airfoil shape modification, tunable lenses and mirrors, and linear vibratory actuators. 


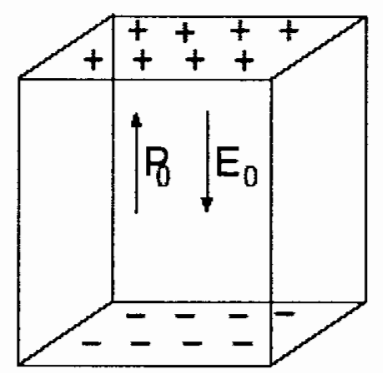

(a)

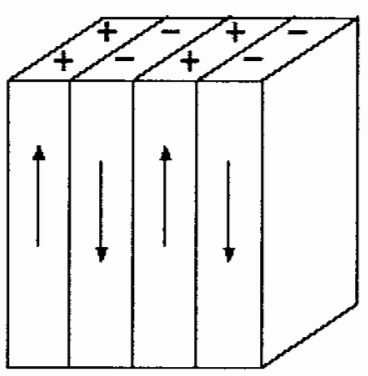

(b)

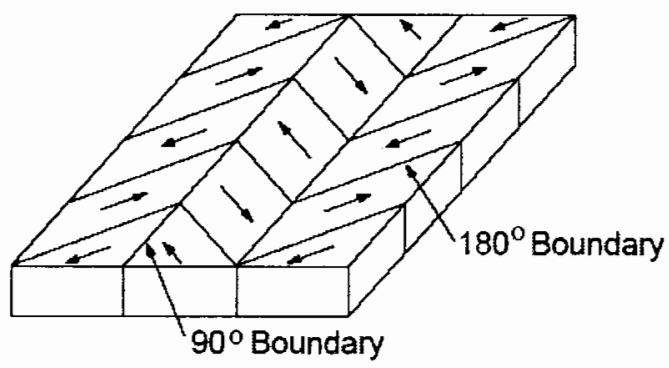

(c)

Figure 2.3: (a) Surface charge due to a spontaneous polarization, (b) twinned $180^{\circ}$ domains which form to minimize electrostatic energy and (c) twinned $90^{\circ}$ and $180^{\circ}$ domain structure for $\mathrm{BaTiO}_{3}$.

\subsubsection{Magnetostrictive Materials}

Magnetostrictive materials are ferromagnetic materials that experience a change in dimensions when magnetized. The corresponding strain in the material is known as magnetostriction. During the first half of the 20th century, applications of magnetostriction included telephone receivers, torque sensors, horns and scanning sonar. The materials used for these early applications typically were nickel, cobalt and alloys of these compounds which are able to produce magnetostrain of approximately 


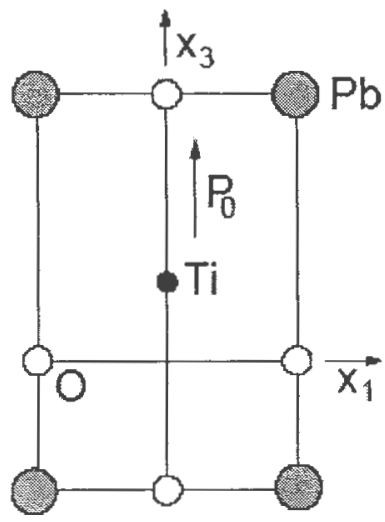

(a)

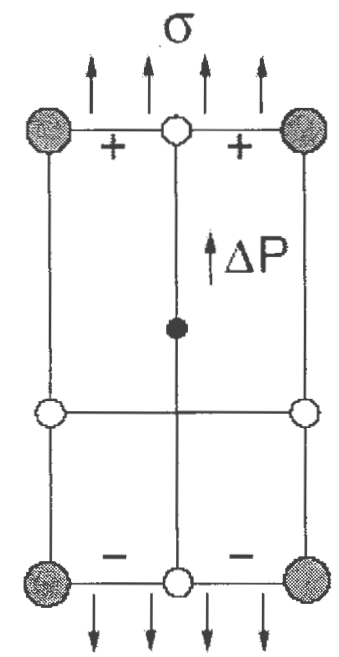

(c)

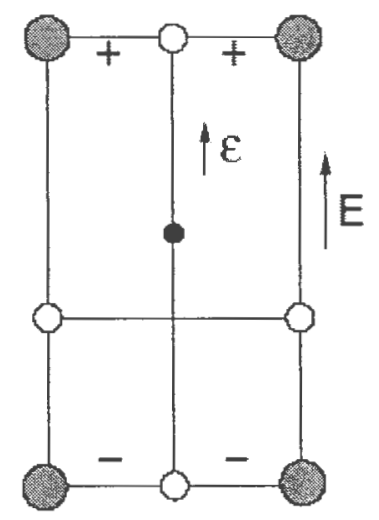

(b)

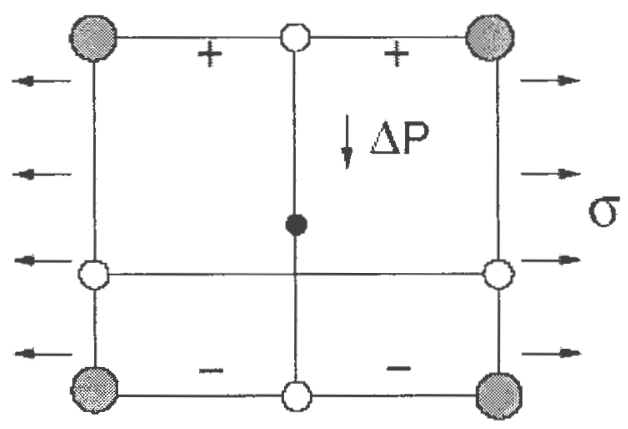

(d)

Figure 2.4: (a) Tetragonal form of $\mathrm{PbTiO}_{3}$ and spontaneous polarization $P_{0}$, (b) indirect piezoelectric effect and direct piezoelectric effects (c) and (d).

$50 \mu \epsilon$. [33] Although magnetostriction is observed in all ferromagnetic materials, certain rare earth alloys exhibit "giant" magnetostriction in excess of $1000 \mu \epsilon$. TerfenolD, first created by the Naval Ordinance Laboratory, is a rare earth compound of 
terbium, iron and dysprosium $\left(\mathrm{Te}_{0.3} \mathrm{Dy}_{0.7} \mathrm{Fe}_{1.9-1.95}\right)$. As the best established commercially available magnetostrictive material, Terfenol-D can produce static magnetostrain of $1600 \mu \epsilon$ at a moderate saturation field of approximately $160 \mathrm{kA} / \mathrm{m}$, but even larger magnetostrictive strains up to $3600 \mu \epsilon$ are attainable when Terfenol-D is employed in transducers driven at resonance [7]. Other rare earth alloys exhibit larger magnetostrains than Terfenol-D but do so exclusively at cryogenic temperatures [8].

The changes in magnetization due to magnetic fields can be either reversible or irreversible. Reversible magnetizations are essentially conservative in that under application of small field increments the magnetization of the material returns to its original position when the field is removed. Irreversible magnetizations due to larger magnetic fields are dissipative in that external forces are needed to force the magnetization to its original state once the field is removed. This behavior is better explained through consideration of the magnetic domains in the material. The magnetic moments in magnetostrictive materials change from their energetically favorable random orientation to a domain structure when cooled below the magnetic phase transition temperature, also known as the Curie temperature $T_{c}$, as illustrated in figure 2.5. Each domain exists as a collection of atoms whose magnetic moments all align in the same direction and have all of their magnetic moments parallel, thus creating a spontaneous magnetization $M_{s}$. This process is accompanied with a spontaneous magnetostriction $\lambda_{0}$ which is equal to one third of the total magnetostriction $e$,

$$
\lambda_{0}=\frac{e}{3}
$$

With no applied magnetic field, the domains of a magnetostrictive material will approximately have random orientation and the total magnetization $M$ will be zero, Figure 2.5(b). When a low-level magnetic field is applied in a given direction, the 
magnetic domains that are favorably aligned with the field grow at the expense of other domains due to domain wall motion, Figure 2.5(c). Removal of the small applied field leads to the domains returning to their original state, thus illustrating the reversible behavior of the material. Application of a strong magnetic field leads to a saturating effect whereby domain rotation along with domain wall motion occurs as the spontaneous moments of each domain align with the applied field, Figure 2.5(d). The difference between the total and spontaneous magnetostriction is the is defined as the saturation magnetostriction and is,

$$
\lambda_{s}=\frac{2}{3} e .
$$

Typical magnetization and strain responses for Terfenol-D are shown in Figure 2.6. At low level fields, the magnetization and strain response are approximately linear. Hysteresis is always present to some extent in Terfenol-D especially when driven at high field levels. Due to the domain wall motion across pinning sites, magnetostrictive materials experience an irreversible process which accounts for the magnetic hysteresis observed [8].

The magnetostriction of Terfenol-D is highly nonlinear and hysteretic as shown in the magnetization $(M-H)$ and total strain $(\epsilon-H)$ in Figure 2.6. In crystalline materials the magnetic moments tend to point along preferred crystallographic directions and do not rotate freely in response to applied fields. This phenomenon is known as magnetocrystalline anisotropy. The anisotropic energy is the required energy to rotate the magnetic moments away from their preferred direction and is closely related to linear magnetostriction. Overcoming the anisotropic energy with the application of a magnetic field results in nonlinear and often discontinuous behavior in magnetization and magnetostriction. Complex crystalline structures, such as Terfenol-D whose 
(a)

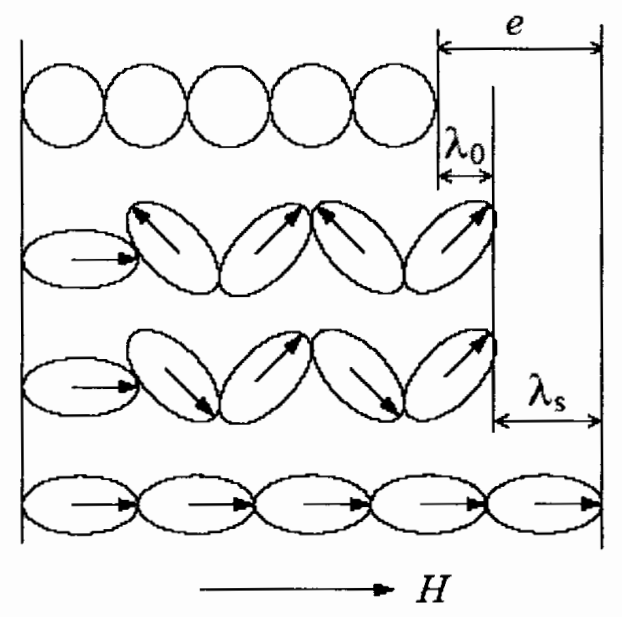

Figure 2.5: Linear magnetostriction illustrated by the state of magnetic domains: (a) isotropic material in the disordered paramagnetic phase above $T_{c}$ modeled with spheres, (b) ellipsoids representing the order due to the spontaneous magnetization of the ferromagnetic phase once cooled through its Curie temperature with no applied field $H$, (c) reversible magnetic moment alignment with an applied magnetic field $H$, and (d) irreversible domain wall rotation from a saturating magnetic field $H$. The spontaneous and saturation magnetostriction $\lambda_{0}$ and $\lambda_{s}$ and total strain $e$ due to the orientation of domains in the field direction are noted.

crystals are grown in dendritic twin sheets along the [11 $\overline{2}]$ direction as shown in Figure 2.7, are often assumed in operating regimes where stress anisotropy dominates crystal anisotropy [7]. The large positive magnetostriction of Terfenol-D is in the $\langle 111\rangle$ direction and a sufficiently large compressive stress in the [112] direction will produce a significant decrease in the internal energy of the crystal at right angles (i.e. $\langle 111\rangle$ direction) to the applied stress. This compressive stress will raise the elastic energy above the intrinsic crystal anisotropic energy, shifting the preferred domains to the $\langle 111\rangle$ magnetic easy axes, and is illustrated for a Terfenol-D rod in Figure 2.8 in which the axial direction is the $[11 \overline{2}]$ direction. The output of the Terfenol-D rod is optimized in this manner by allowing for efficient 90 degree (maximum) rotation 


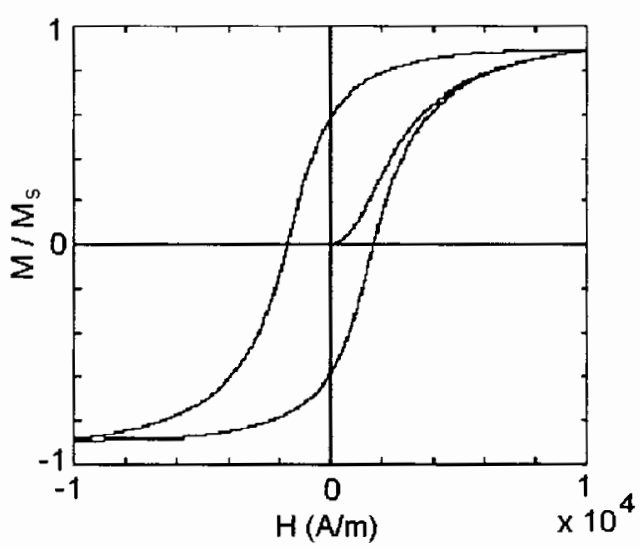

(a)

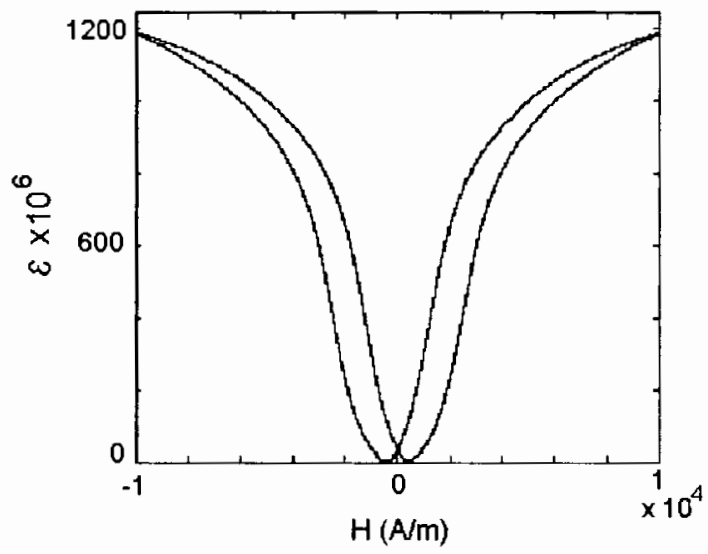

(b)

Figure 2.6: (a) Relative magnetization $M / M_{s}$ and (b) magnetostriction $\epsilon$ produced by a sinusoidal magnetic field $H$ for Terfenol-D.

of the domains and thus minimizing the saturation magnetic field [33, 9]. Compression of Terfenol-D also ensures the safety of the specimen. Terfenol-D is weak in tensile strength and operation under compression assures that the material is never in tension.

The direction of the magnetic moment is coupled to the interatomic spacing between atoms. During domain wall motion and magnetic moment rotation, the interatomic spacing between atoms changes causing the material to deform. Linear or Joule magnetostriction refers to this change in length exhibited by magnetic materials when exposed to magnetic fields and is phenomenologically similar to the inverse piezoelectric effect. Figure 2.5 illustrates the magnetostriction in the disorganized paramagnetic regime, ferromagnetic regime demagnetized and the ferromagnetic regime magnetized to saturation. Applications arising from the Joule magnetostriction mechanism include sonar transducers, linear and rotational motors, and 


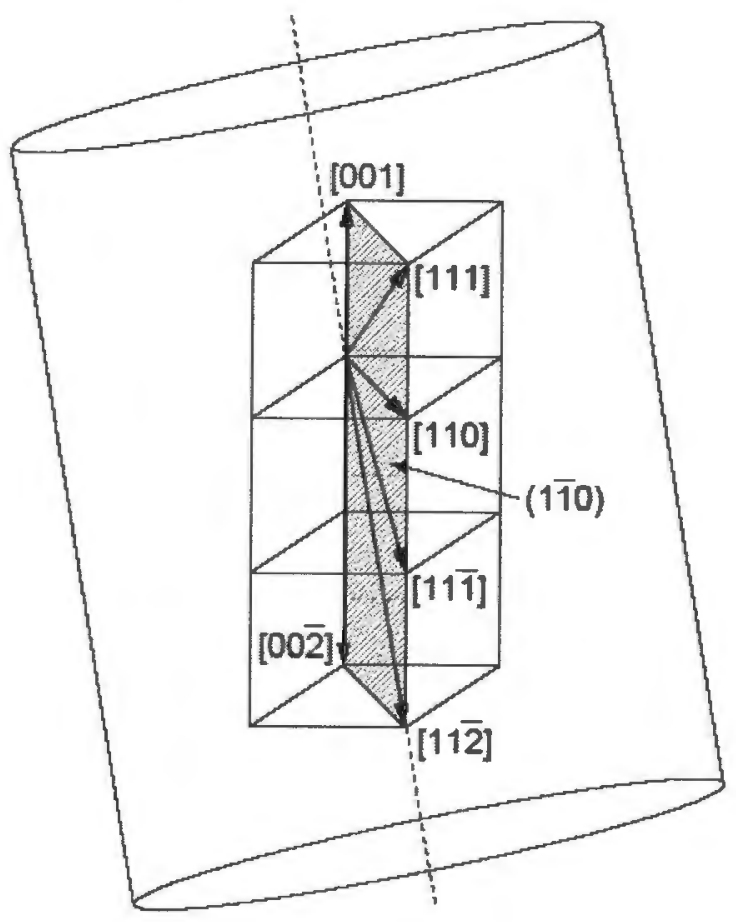

Figure 2.7: Crystallographic orientations in monolithic Terfenol-D.

hybrid magnetostrictive/piezoelectric devices. Figure 2.9(a) is a rotational motor that uses a piezoelectric material to clamp the steel disk while the dual magnetostrictive rods translate the clamp tangentially causing rotation [25] while (b) performs a similar task by using two magnetostrictive elements whose tip has an elliptical track [1]. Figure 2.10 illustrates various magnetostrictive sonar transducers. Given the inductive nature of magnetostrictive materials and capacitive nature of piezoelectric materials, combinations of these materials in a hybrid device creates a resonant electrical circuit. Figure 2.10(d) is a sonar projector whose inherent 90 degree phase shift between the magnetostrictive and piezoelectric elements is used to increase the bandwidth. 
[11̄]

(a)

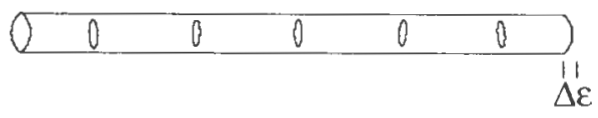

(c)

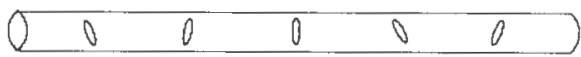

(b)

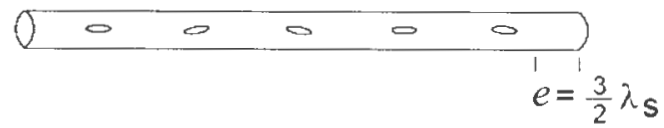

(d)

Figure 2.8: Magnetic domains in a Terfenol-D rod with crystallographic alignment (a); (b) Orientation of unstressed rod without an applied magnetic field. (c) Orientation of pre-stressed rod with no applied field and strain $\Delta \epsilon$ due to compression. (d) Orientation of pre-stressed rod when field is applied in the axial direction of the rod.

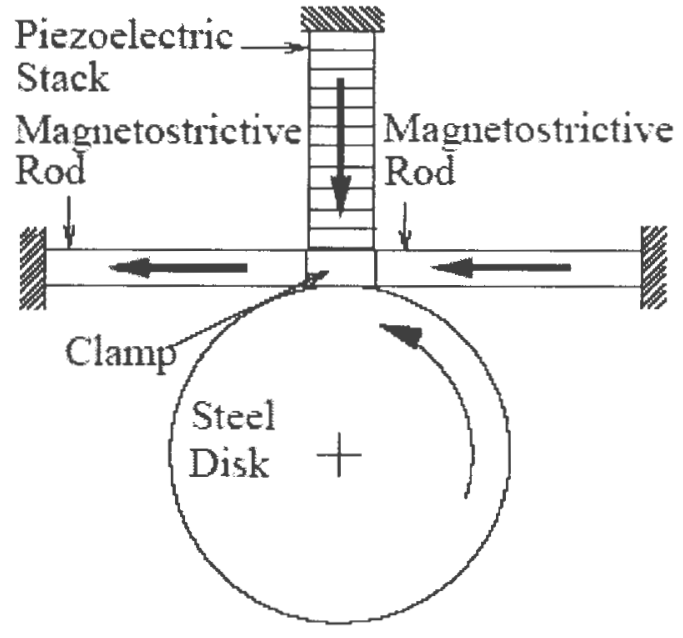

(a)

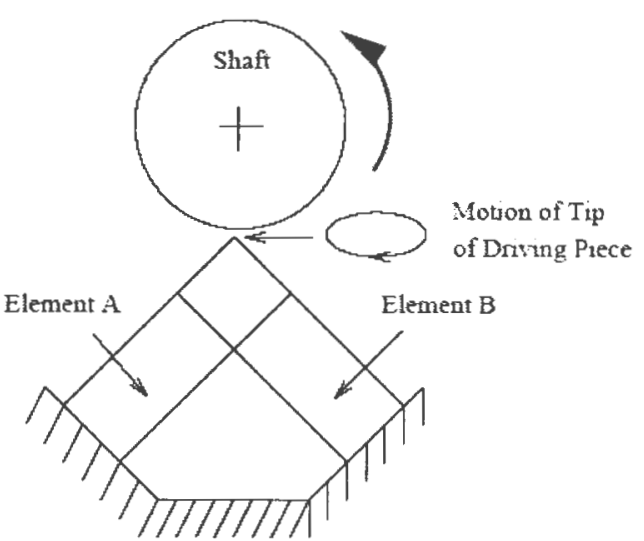

(b)

Figure 2.9: Magnetostrictive rotary transducers: tive/piezoelectric type [25] and (b) elliptical type [1].

(a) hybrid magnetostric-

In the reciprocal behavior, known as the Villari effect, stresses in the material produce magnetization. This mechanism is phenomenologically analogous to the direct 


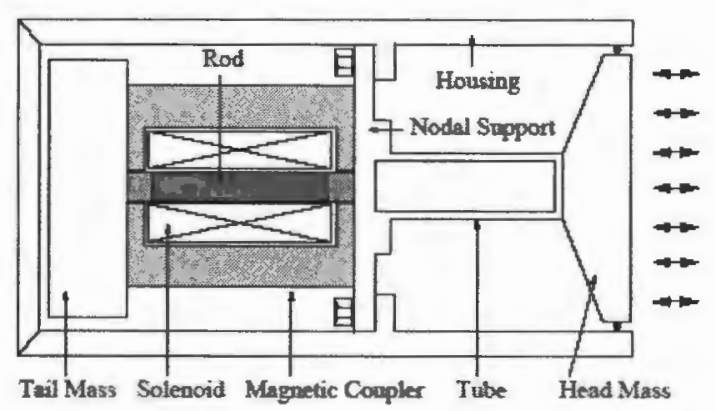

(a)

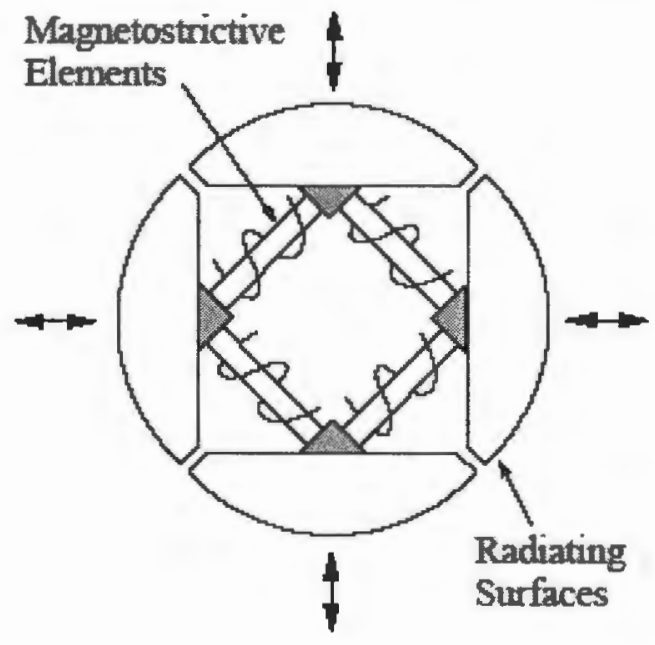

(c)

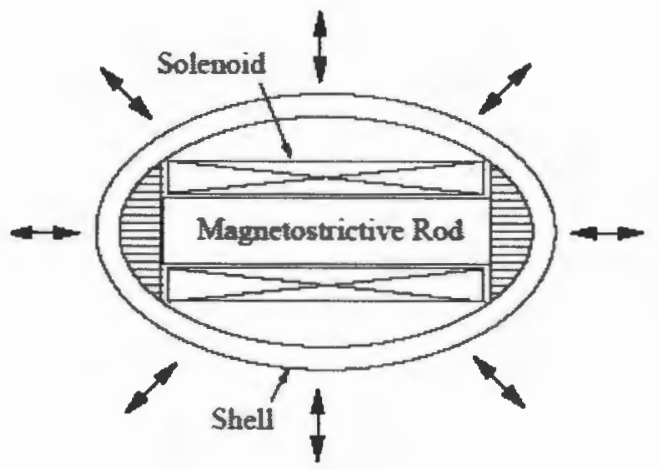

(b)

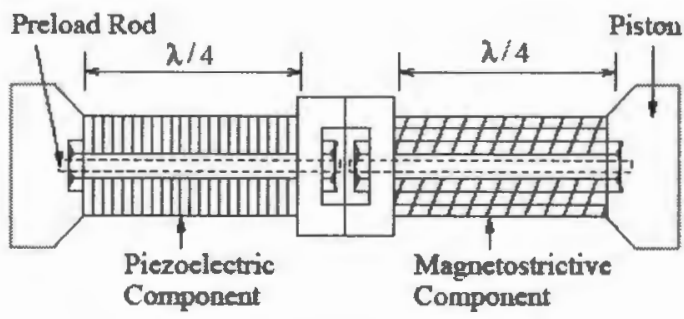

(d)

Figure 2.10: Magnetostrictive sonar transducers: (a) Tonpilz [7], (b) flextensional, (c) square ring type and (d) hybrid magnetostrictive/piezoelectric type [5].

piezoelectric effect and can be used in sensor applications including torque, waveguide position, force and stress sensors.

Because under the application of positive and negative magnetic fields the strain produced by Terfenol-D is always positive, as in Figure 2.6, the frequency of the strain is twice that of the applied field. However, bidirectional strains are achievable when 
Terfenol-D is magnetically biased. In this case, the coupled behavior can be approximated by the linearized piezomagnetic constitutive equations relating the strain $\epsilon$ and magnetic induction $B$ with stress $\sigma$ and magnetic field $H$,

$$
\begin{aligned}
\epsilon & =s^{H} \sigma+q H \\
B & =q^{*} \sigma+\mu^{\sigma} H .
\end{aligned}
$$

Here, $s^{H}$ is the compliance of Terfenol-D at constant field, $q=q^{*}$ is a symmetric magnetoelastic coupling coefficient, and $\mu^{\sigma}$ is the permeability at constant stress [11, 33]. Hysteresis and nonlinearities found at larger drive levels are not represented by equations (2.5)-(2.6). More advanced modeling techniques are detailed in $[6,11,8$, $33]$.

\subsubsection{Smart Material Pumps}

Smart material transducers produce large forces and can do so over large bandwidths but at the expense of small displacements. Smart material pumps utilizing piezoelectric or magnetostrictive materials use hydraulic advantage by transforming the high force and low velocity output of the smart material into high pressure and pseudo-continuous volume flow rate. Many pump designs and rectification techniques are available as outlined in the following paragraphs.

Piezoelectric stack actuators have static strains in the range of $0.10 \%$ to $0.15 \%$ and are rigid enough for this type of application. Several piezoelectric pumps have been constructed that rely on standard mechanical/passive check valves $[2,26]$. These check valves limit the frequency of operation to approximately $200-400 \mathrm{~Hz}$. Mauck and Lynch present a PZT driven piezopump with optimal performance occurring at a drive voltage of $800 \mathrm{~V}$ and $60 \mathrm{~Hz}$ [24]. The corresponding no-load actuation 
rate of a $3 / 8$ in bore cylinder is $7.25 \mathrm{~cm} / \mathrm{sec}$ and a blocked force of $271 \mathrm{~N}$ (61 lbs). Sirohi and Chopra compared the operation of the pump with standard ball and cone check valves to custom made reed valves and concluded that the reed valves allow for increased pumping frequencies up to $1 \mathrm{kHz}$ with a maximum volume flow rate occurring at $300 \mathrm{~Hz}$ [32]. The present form of the piezopump is more suited at quasistatic applications. Chapman et al. developed three small piezo-hydraulic pumps with the smallest weighing only 90 grams and capable of 600 psi of blocked pressure and $45 \mathrm{cc} / \mathrm{min}$ at $60 \mathrm{~Hz}$ [14]. A blocked pressure differential of $250 \mathrm{psi}$ and a volume flow rate of $338 \mathrm{cc} / \mathrm{min}$ were obtained at $400 \mathrm{~Hz}$ for a larger piezoelectric pump utilizing reed valves. Piezoelectric unimorph discs are used as active valves in a piezoelectrichydraulic pump developed by Lee et al. [22]. A bandwidth of $15 \mathrm{kHz}$ is obtained for the pump without fluid loading. A prototype pump, illustrated in Figure 2.11, produces with a volume flow rate of $3.4 \mathrm{cc} / \mathrm{sec}$ and a stall pressure of $8.3 \mathrm{MPa}(1200 \mathrm{psi})$ with a pump specific power density of approximately $12 \mathrm{~W} / \mathrm{kg}$. Magnetorheological (MR) fluid has been used in operation with a piezohydraulic actuator by Yoo, Sirohi and Wereley $[35,34]$. The magnetorheological piezoelectric actuation system utilzes four MR valves assembled in a Wheatstone bridge configuration to control the direction of a hydraulic cylinder.

Recent years have seen significant advances in smart material actuators. A prototypical Terfenol-D driven linear actuator is illustrated in Figure 2.12. Gerver et al. [23] developed a magnetostrictive water pump capable of producing a flow rate of $30 \mathrm{~mL} / \mathrm{sec}$ and a pressure of 5 psi. A Terfenol-D driver is connected to a hydraulic amplifier, consumes $25 \mathrm{~W}$ of power and was developed for space applications. 


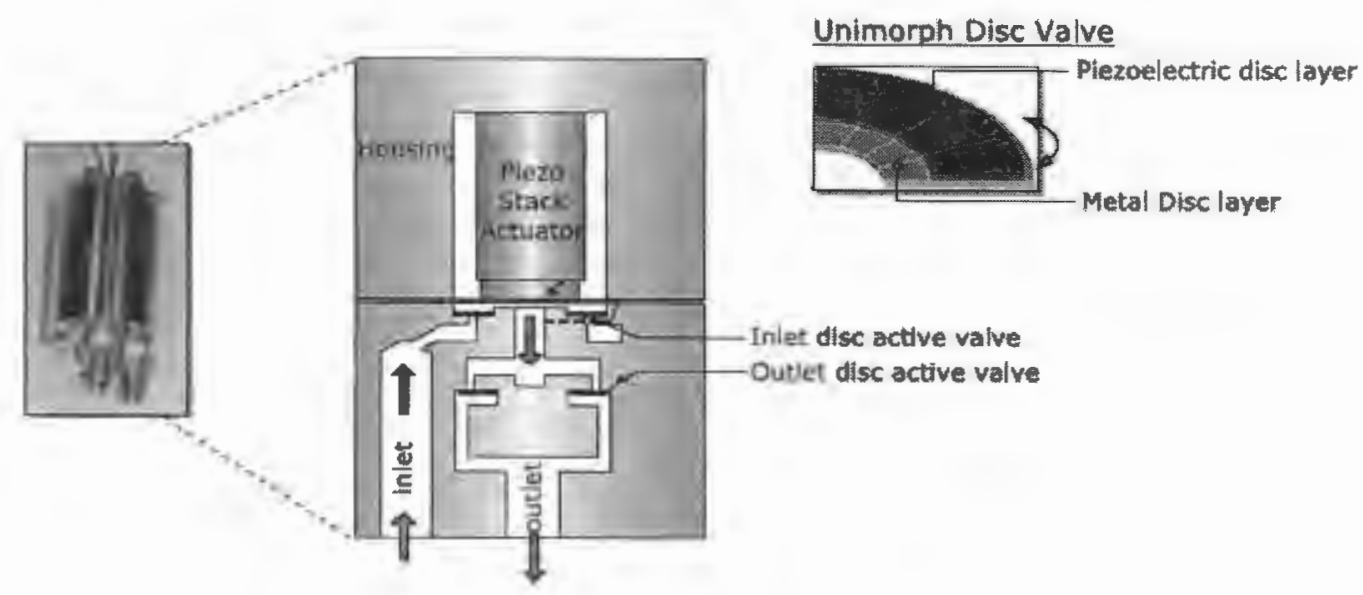

Figure 2.11: Schematic of piezoelectric-hydraulic pump with active unimorph disk valves to control flow at inlet and outlet [22].

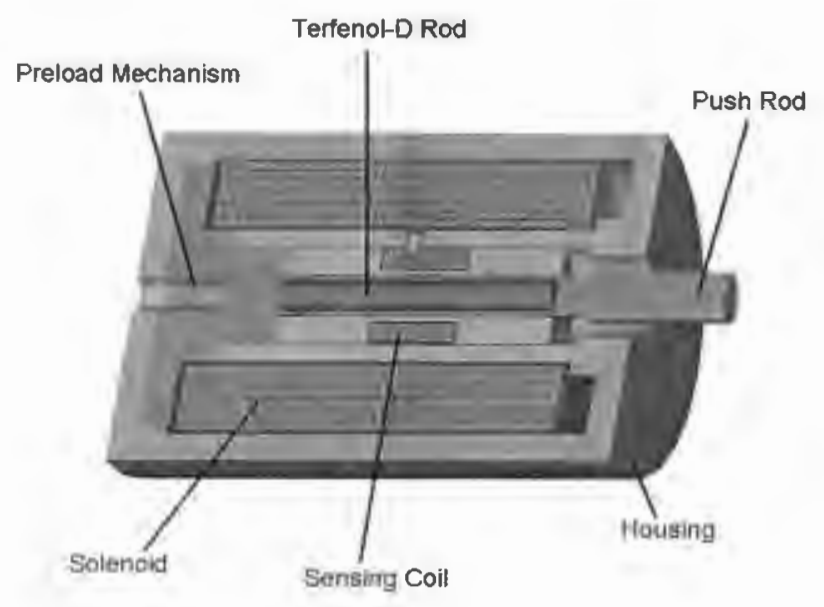

Figure 2.12: Solid model of a typical Terfenol-D transducer design.

Bridger et al. present the development of a magnetostrictive pump capable of producing 3000 psi with an electromechanical coupling of $60 \%$ [4]. The pump relies on 
the resonant motion of an inertial mass to create the pressure. The magnetostrictive pump was developed to be mated with a Terfenol-D driven smart valve/actuation system being developed at Moog, Inc [4]. Nosse and Dapino present an innovative MR valve system for rectification of magnetostrictive vibrations [27]. The MR valve system is a double sided, dynamic valve that can fully close and thus fully block the volume flow.

Advantages of magnetostrictive materials over piezoelectric materials include the ability to magnetically bias the magnetostrictive material resulting in lower voltages at quasi-static frequencies and a lower Young's modulus, which is advantageous for producing low frequency resonators compact in size. The ability of magnetostrictive materials to operate under large prestress and low stiffness enables larger dynamic strains at resonance than piezoelectric materials. Larger strain leads to larger pressure and volume flow rate outputs in smart material pumps. Piezoelectric materials require a DC voltage to center the material which leads to input voltages an order of magnitude larger than the input voltages for magnetostrictive materials but have a low power consumption because of their capacitative nature and high output energy per mass unit. Terfenol-D actuators require a coil to induce the magnetic field in the material which leads to larger actuator diameters than piezoelectric actuators. Although advantages exist for both magnetostrictive and piezoelectric materials as the driving elements for EHAs, the smart material pump presented in this thesis is constructed with Terfenol-D as the driving element. 


\section{CHAPTER 3}

\section{LINEAR DYNAMIC SYSTEM MODEL}

The fluid network associated with the magnetostrictive pump being investigated in this thesis is represented by the schematic in Figure 3.1. A dynamic system model which describes the relationships between the electrical, mechanical and fluid subsystems of the Terfenol-D transducer is presented. The model characterizes the pump in two ways: (i) system operation, i.e., system pressure and actuator velocity and displacement, is described for a given harmonic input and (ii) dynamic characteristics of the Terfenol-D pump, i.e., various parameters affecting the electrical drive-point impedance, are developed through electrical impedance analysis. First, the coupled equations of motion for the mechanical and fluid subsystems are derived separately. The differential equations for each subsystem are then assembled into a system matrix and state space techniques are utilized to solve the system matrix for a given harmonic input. These solutions characterize the harmonic aspects of the pump. Last, an expression is developed for the electrical drive-point impedance relating the electrical and mechanical domains through manipulation of electroacoustic transduction equations for electromechanical transducers and piezomagnetic constitutive 


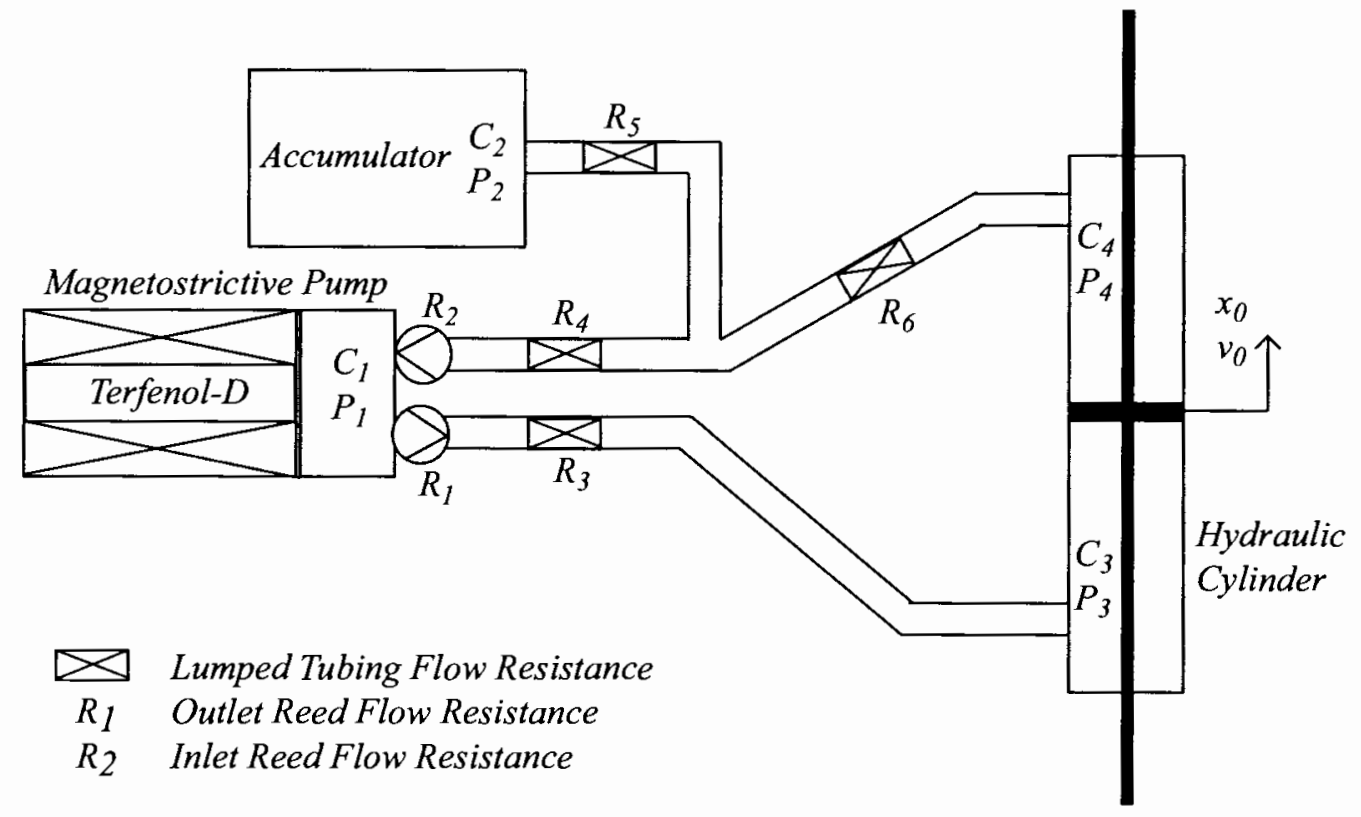

Figure 3.1: Schematic illustrating the fluid network of the Terfenol-D transducer while hydraulically attached to a piston.

equations. The electroacoustic portion of the dynamic system model uses a superposition of results obtained from the harmonic portion of the model along with an evaluation of the electrical impedance in order to obtain the desired characteristics.

\subsection{Mechanical Model of Terfenol-D Pump}

In order to take full advantage of its magnetomechanical effects, Terfenol-D must be utilized in a transducer comprising a magnetic circuit and mechanical preload. The Terfenol-D transducer is similar to typical Terfenol-D transducer designs, as in Figure 2.12, in that the pushrod acts as a piston by pushing against a thin stainless steel diaphragm and producing volume flow. Terfenol-D provides the mechanical stiffness and damping of this system and generates the mechanical driving force through 
magnetostrictive vibrations. The stiffness of Terfenol-D is related to the Young's modulus $E_{y}^{H}$ and the geometry of the rod,

$$
k_{t}=\frac{E_{y}^{H} A_{t}}{L_{t}}
$$

where $A_{t}$ is the cross-sectional area and $L_{t}$ is the length of the rod. The damping coefficient is more difficult to define but is clearly evident as the inherent hysteresis in Terfenol-D transducers is due to mechanical losses. Since the Terfenol-D transducer is coupled to a hydraulic circuit, there are fluid stiffness, damping and inertia effects that must be taken into account.

The Terfenol-D transducer subsystem is modeled as a second order, two degree of freedom spring-mass-damper system as illustrated in the block diagram in Figure 3.2(a). Figure 3.2(b) illustrates the orientation of the Terfenol-D rod in the transducer which is the basis for the block diagram. The column of fluid is contacting both the piston and the pumping chamber wall and is thus fixed with respect to these two planes. The vibratory model accounts for the mechanical stiffness and damping coefficients of Terfenol-D as well as the fluid damping, stiffness and inertance of the hydraulic oil in the pumping chamber. The two degrees of freedom arise from motion of the effective mass of the Terfenol-D rod, $m_{e f f}$, and the mass of the column of fluid $m_{f}$. The effective mass includes the dynamic mass of the Terfenol-D rod and mass of the piston and diaphragm. The mechanical stiffness and damping of the Terfenol-D transducer behave as the parallel combination of a spring $k_{i}$ and damper $b_{i}$. It is noted that $k_{i}=k_{t}+k_{d}$ and $b_{i}=b_{t}+b_{d}$, where the subscripts refer to Terfenol-D and the stainless steel diaphragm respectively. The fluid stiffness $k_{f}$ and damping $b_{f}$ are modeled as described in Figure 3.2(a) in order to account for the fluid contact with the piston and chamber wall. The two forces on the subsystem are the 
force generated by the magnetostrain of the Terfenol-D element, $\alpha I$, which is related to the input current through the electroacoustic relations described in Section 3.5, and the force applied to the mass of the fluid due to the pressure inside the pumping chamber, $F_{e x t}=P_{1} A_{i}$ where $A_{i}$ is the cross-sectional area of the pumping chamber. The magnetostrictive subsystem is coupled to the fluid domain through the pressure $P_{1}$. The equation of motion of each mass is solved by using Newton's second law and written in terms of the velocity and displacement state variables $\left(x_{t}, v_{t}, x_{f}, v_{f}\right)$,

$$
\begin{aligned}
m_{e f f} \dot{v}_{t}+\left(b_{i}+\frac{b_{f}}{2}\right) v_{t}-\frac{b_{f}}{2} v_{f}+\left(k_{i}+\frac{k_{f}}{2}\right) x_{t}-\frac{k_{f}}{2} x_{f} & =\quad \alpha I \\
m_{f} \dot{v}_{f}-\frac{b_{f}}{2} v_{t}+b_{f} v_{f}-\frac{k_{f}}{2} x_{t}+k_{f} x_{f}=-F_{e x t}= & -P_{1} A_{i} .
\end{aligned}
$$

where the fluid stiffness is defined by the bulk modulus of the hydraulic fluid, $\beta$, the cross-sectional area of the pumping chamber and the height $h$ of the pumping chamber,

$$
k_{f}=\frac{\beta A_{i}}{h}
$$

In order to characterize of the Terfenol-D transducer, it is necessary to quantify the effects of loading on the behavior of the transducer. Changes in the fluid loading will affect the dynamic characteristics of the Terfenol-D transducer. As a means to fully describe the mechanical regime of the transducer, the transfer function of force per mass velocity, i.e. the mechanical impedance, can be found for the Terfenol-D portion of the transducer. In order to quantify the mechanical impedance, the external loading due to the fluid is assumed zero and the force per Terfenol-D velocity is shown to be

$$
Z_{\text {mech }}=\frac{\alpha I}{v_{t}}=\frac{m_{e f f}(j \omega)^{2}+b_{i} j \omega+k_{i}}{j \omega}
$$




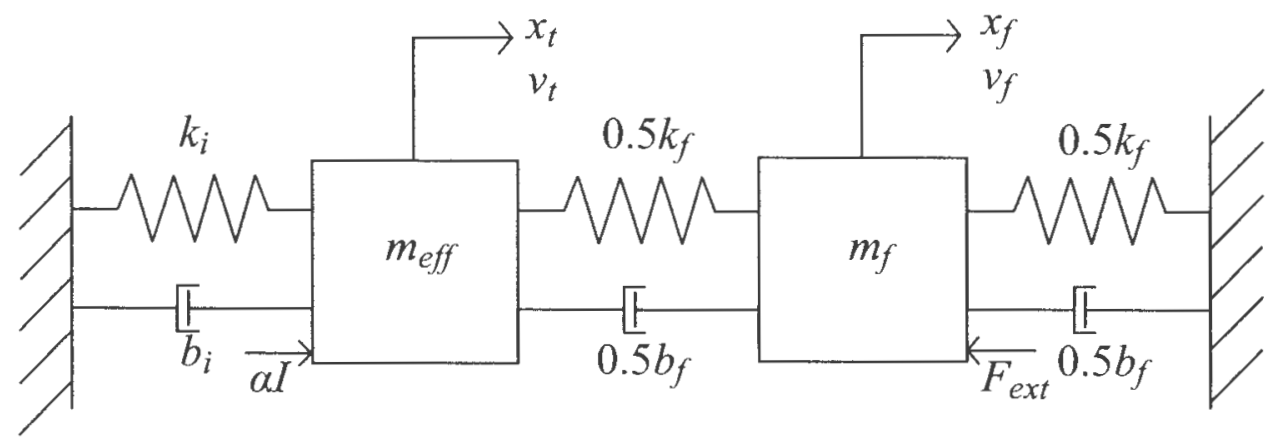

(a)

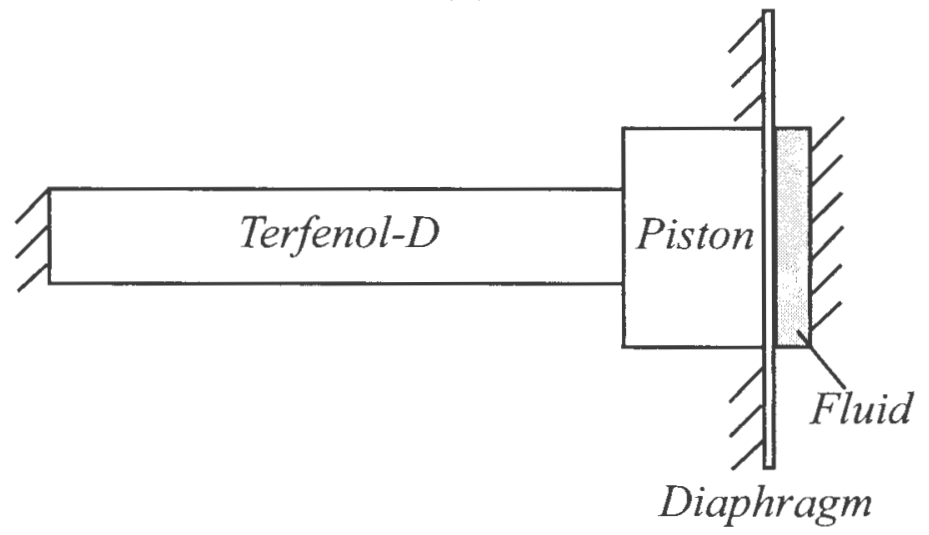

(b)

Figure 3.2: (a) Two degree of freedom vibratory model of the magnetostrictive pump and (b) orientation of Terfenol-D inside the transducer.

The fluid portion removed in this calculation is defined as the load impedance of the transducer and is described in greater detail in Section 3.5.4.

\subsection{Fluid Subsystem}

The fluid network shown in Figure 3.1 is used to derive the equations of motion that govern the fluid flow through the pump and fluid network. The oscillatory fluid flow inside the pumping chamber produced by the magnetostrictive pump is rectified by custom-made reed valves. The reed valves are stainless steel flaps that can only 


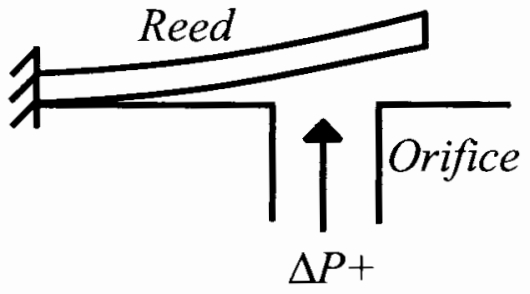

(a)

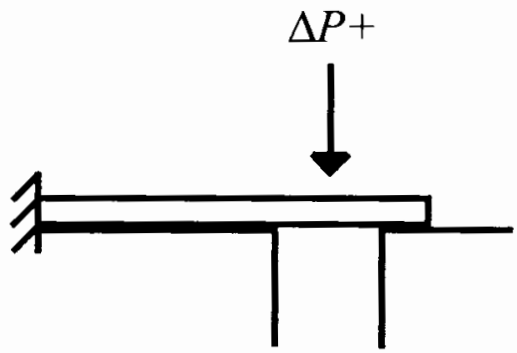

(b)

Figure 3.3: A cross-sectional view of the reed valve, illustrating its operation. (a) For a positive pressure gradient in the desired direction of fluid flow, the reed flap deflects and allows fluid flow. (b) For a positive pressure gradient in the opposite direction, the reed flap blocks the orifice and allows no flow.

deform in the direction of fluid flow. Figure 3.3 illustrates the reed valve operation. A positive pressure gradient across the reed valve deforms the reed and allows flow. A negative pressure gradient pushes the reed against the orifice and effectively blocks flow in the direction toward the piston. This behavior can be represented as a finite fluid resistance in the direction of allowed flow and infinite fluid resistance otherwise. An accumulator is placed on the low pressure side of the pump and provides a bias pressure on the fluid. The bias pressure prevents fluid cavitation and supplies the pumping chamber with fluid after each stroke of the Terfenol-D transducer. The hydraulic cylinder transmits the force produced by the magnetostrictive pump to an external mechanical load. A lumped parameter analysis is used to derive the fluid differential equations of the four pressure states in the hydraulic circuit $\left(P_{1}, P_{2}, P_{3}\right.$, $\left.P_{4}\right)$. The elemental equations for ideal fluid capacitors and flow resistors are described 
in terms of pressure and volume flow rate,

$$
\begin{aligned}
Q_{c} & =C_{f} \frac{d P_{1 r}}{d t} \\
P_{12} & =R_{f} Q_{R},
\end{aligned}
$$

with fluid capacitance $C_{f}$, flow resistance $R_{f}$, pressure drops $P_{1 r}$ and $P_{12}$ and corresponding volume flow rates $Q_{c}$ and $Q_{R}$ as described in Figure 3.4 [31]. The fluid interconnection laws of continuity and compatibility, analogous to Kirchoff's laws for electrical circuits, are used to derive the differential equations for the pressure state variables. The law of continuity states that the sum of the volume flow rates at any node must be zero and the law of compatibility states that the sum of the pressure drops around a closed loop is zero. There are four fluid capacitors each corresponding to one of the four pressure states and a fluid resistor for each line resistance. The mass of the column of fluid in the pumping chamber is modeled in the mechanical domain; considering the relatively low mass density of the hydraulic fluid, the inertance of the fluid lines is assumed negligible. The capacitance of the fluid lines also is insignificant and is not modeled. The values of the fluid capacitances and flow resistances are determined by,

$$
\begin{aligned}
C_{f} & =\frac{V}{\beta} \\
R_{f} & =\frac{128 \mu_{f} L}{\pi d^{4}}
\end{aligned}
$$

where $V$ is the volume of the fluid cavity, $\beta$ is the bulk modulus of the hydraulic fluid, $\mu_{f}$ is the dynamic fluid viscosity, and $L$ and $d$ are the length and diameter of the fluid line [31].

The velocity of the mass of the fluid provides the input hydraulic flow for the hydraulic circuit. The output cylinder motion is dependent on the pressure states on 


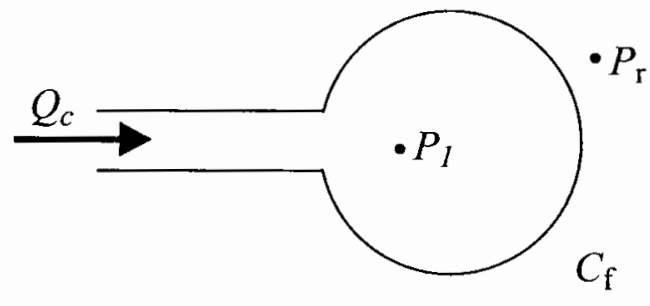

(a)

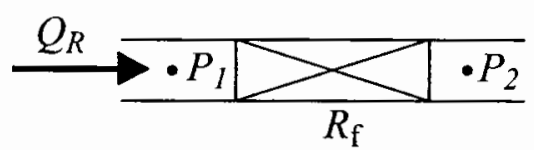

(b)

Figure 3.4: Symbolic diagrams of (a) a fluid capacitor and (b) a fluid resistor. [31]

either side of the piston and is modeled as a single degree of freedom system in terms of the output position and velocity $\left(x_{o}, v_{o}\right)$ of the piston.

\subsection{State Space Modeling of Complete EHA}

State space analysis is used to solve the portion of the dynamic system model coupling the mechanical and fluid domains of the pump. Newton's second law and the fluid interconnection laws are used to derive the coupled mechanical and fluid system of equations for the pump. The mechanical equations of motion include both displacement and velocity state variables. By adding the velocity state variables in the mechanical equations of motion, each second order equation is broken into two first order equations resulting in a complete system of first order equations. This allows simplification of the model in that only one system matrix is needed for obtaining a solution. Since the mechanical and fluid equations of motion are coupled, simultaneous solutions are required. The complete system level model, represented as a matrix state equation with system matrix $[A]$, input matrix $[B]$, state vector $\vec{x}(t)$ 
and forcing vector $\vec{u}(t)$, is solved using the convolution (superposition) integral,

$$
\begin{aligned}
\dot{\vec{x}}(t) & =[A] \vec{x}(t)+[B] \vec{u}(t) \\
\vec{x}(t) & =e^{[A]\left(t-t_{0}\right)} \vec{x}\left(t_{0}\right)+\int_{t_{0}}^{t} e^{[A](t-\tau)}[B] \vec{u}(\tau) d \tau
\end{aligned}
$$

The solution is assumed to consist of two parts: (i) a free component $\vec{x}_{\text {free }}$ which is the solution of the matrix state equation for the forcing vector at time $t=t_{0}$ and initial conditions $\overrightarrow{x_{0}}$, and (ii) a forced component $\vec{x}_{\text {forced }}$ for the forcing input $\vec{u}(t)$ and zero initial state [3]. The free solution accounts for any initial transients in the system while the forced solution accounts for the response to the forcing input alone. The matrix state equation is solved for each time step and uses the previous solution as its initial condition. Since the solution is found using a linear superposition solver, the time step must be small enough so that the changes between time steps can be assumed linear. Time steps that are too large may result in inaccurate and often unstable solutions.

The differential equations of the fluid and mechanical subsystems are derived separately and then combined to form the system matrix $[A]$. The state vector, input vector and system matrix are given in equations (3.12)-(3.16) and are assembled in MATLAB. The harmonic portion of the dynamic system model is solved using the superposition solver 'lsim', which gives the time response of a state space model $[A]$ with a specified input vector $[B] \vec{u}(t)$ and corresponding time vector. An algorithm is written describing the fluid diode behavior of the reed valves. The pressure states across the reed valves determine the value of the reed valve resistance. Since the capacitance of the fluid capacitors $C_{3}$ and $C_{4}$ in the hydraulic cylinder have varying heights with respect to output actuator motion, they depend on the value of the hydraulic cylinder displacement. The values of the reed valve resistances and fluid 
capacitors $C_{3}$ and $C_{4}$ are determined before each solution, depending on the pressure and actuator displacement states.

$$
\begin{aligned}
& \vec{x}(t)=\left[\begin{array}{c}
x_{t} \\
v_{t} \\
x_{f} \\
v_{f} \\
P_{1} \\
P_{2} \\
P_{3} \\
P_{4} \\
x_{o} \\
v_{o}
\end{array}\right] \\
& {[B] \vec{u}(t)=\left[\begin{array}{c}
0 \\
\frac{N q k_{t}}{m_{e f f}} \\
0 \\
0 \\
0 \\
0 \\
0 \\
0 \\
0 \\
0
\end{array}\right] I(t)}
\end{aligned}
$$




$$
\begin{aligned}
& 0 \text { 0 0 0 } 0 \text { o } \\
& 00000000000 \\
& 00000
\end{aligned}
$$

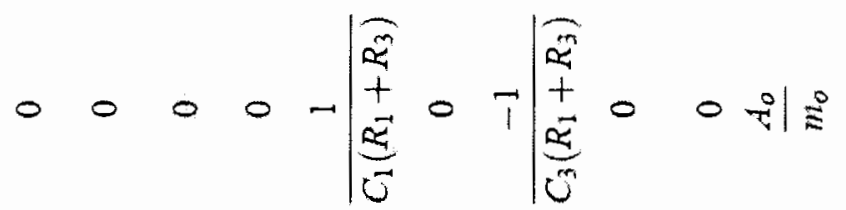

$$
\begin{aligned}
& 0000
\end{aligned}
$$

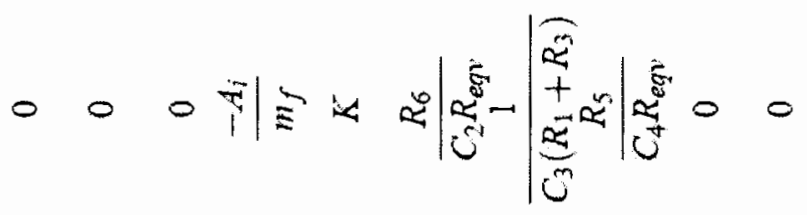

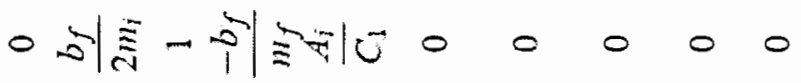

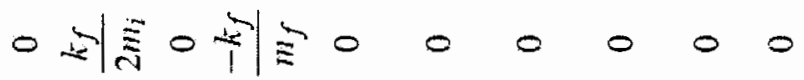

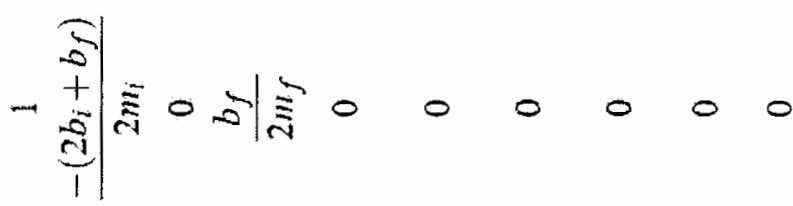

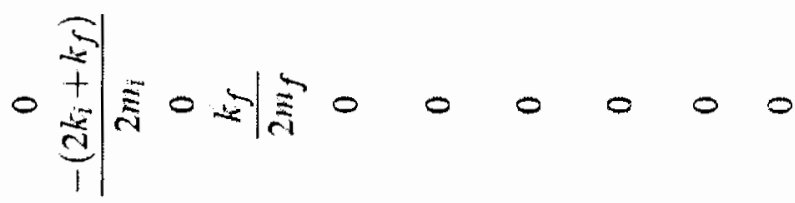

$$
\begin{aligned}
& \frac{11}{\sqrt[3]{3}}
\end{aligned}
$$




$$
\begin{aligned}
R_{e q v} & =\left(R_{2}+R_{4}\right)\left(R_{5}+R_{6}\right)+R_{5} R_{6} \\
K & =\frac{R_{5} R_{6}\left(R_{1}+R_{3}\right)-R_{e q v}\left(R_{1}+R_{2}+R_{3}+R_{4}\right)}{C_{1} R_{e q v}\left(R_{1}+R_{3}\right)\left(R_{2}+R_{4}\right)}
\end{aligned}
$$

\subsection{Linear Magnetostrictive Modeling}

Although Terfenol-D is nonlinear and hysteretic, a set of linearized equations accurately describes the response for low to moderate applied fields. These relations are the combination of elastic, magnetic and thermal effects on the strain $\epsilon$ and magnetic induction $B$. They are given in differential form as,

$$
\begin{aligned}
d \epsilon & =\left(\frac{\partial \epsilon}{\partial \sigma}\right)_{H, T} d \sigma+\left(\frac{\partial \epsilon}{\partial H}\right)_{\sigma, T} d H+\left(\frac{\partial \epsilon}{\partial T}\right)_{H, \sigma} d T \\
d B & =\left(\frac{\partial B}{\partial \sigma}\right)_{H, T} d \sigma+\left(\frac{\partial B}{\partial H}\right)_{\sigma, T} d H+\left(\frac{\partial B}{\partial T}\right)_{H, \sigma} d T
\end{aligned}
$$

where $H$ is the applied magnetic field, $T$ is the absolute temperature and $\sigma$ is the axial stress. Neglecting the temperature effects and expressing the partial derivatives as real coefficients results in the equations $(2.5)-(2.6)$,

$$
\begin{aligned}
\epsilon & =s^{H} \sigma+q H \\
B & =q^{*} \sigma+\mu^{\sigma} H,
\end{aligned}
$$

In equation (3.19) the strain represented by the superposition of Hooke's law $\sigma=\epsilon E_{y}^{H}$ and the magnetostriction $\lambda=q H$. In equation (??) the magnetic induction is the superposition of a magnetic term $B=\mu H$ and a mechanically coupled term $q^{*} \sigma$. It is often assumed that $q=q^{*}$. These piezomagnetic constitutive equations are used next in Section 3.5 in the development of the dynamic aspects of the model. 


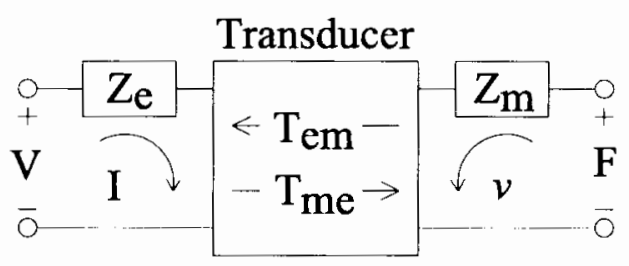

Figure 3.5: Schematic representation of an electromechanical system. [17]

\subsection{Electroacoustics Theory}

In describing the coupling between the electrical and mechanical domains of the Terfenol-D transducer, classical electroacoustic transduction theory is considered, assuming linear transducer operation [17]. Figure 3.5 illustrates a transduction model for general electromechanical transducers. This model is used to derive two equations that couple the electrical and mechanical domains,

$$
\begin{aligned}
& V=Z_{e} I+T_{e m} v \\
& F=T_{m e} I+Z_{m} v,
\end{aligned}
$$

where $V$ is the applied voltage to the transducer, $I$ is the current flow through the transducer, $F$ is the force on the transducer, $v$ is the velocity, $Z_{e}$ and $Z_{m}$ are the blocked electrical and mechanical impedances, and $T_{e m}$ and $T_{m e}$ are transduction coefficients that describe the electromechanical coupling. The subscripts em and me refer to 'electrical due to mechanical' and 'mechanical due to electrical' energy transduction processes. The blocked electrical impedance is the measured impedance that occurs when the output motion of the Terfenol-D is completely blocked.

Important transducer characteristics are revealed by studying the electrical drivepoint impedance which is defined as the complex ratio of voltage to current. In typical 
transducer applications described by equations (3.21)-(3.22), the force $F$ is connected to a mechanical load with impedance $Z_{L}$ defined as

$$
F \equiv-Z_{L} \dot{x_{t}}
$$

The definition of load impedance is substituted into equation (3.22) and the velocity is written as,

$$
v=\frac{T_{m e} I}{Z_{m}+Z_{L}}
$$

The velocity relating the blocked mechanical and load impedances is then and substituted into equation (3.21) in order to find an expression for the electrical drive-point impedance $Z_{e e}$,

$$
Z_{e e}=\frac{V}{I}=Z_{e}+\frac{-T_{e m} T_{m e}}{Z_{m}+Z_{L}}=Z_{e}+Z_{m o t}
$$

The electrical-drive point impedance is the sum of the blocked electrical impedance $Z_{e}$ and motional impedance $Z_{\text {mot }}$ which quantifies the electromechanical coupling of the transducer. Figure 3.6 shows the magnitude of the electrical drive-point impedance for an Etrema Terfenol-D motor with a 4 in long/0.5 in diameter Terfenol-D rod and illustrates the mechanical contributions to the electrical impedance. The solid line shows the blocked electrical impedance $Z_{e}$ while the coupled motional effects of $Z_{\text {mot }}$ account for the deviation from this line.

\subsubsection{Electrical Impedance Analysis}

Analysis of the electrical drive-point impedance provides a way of determining fundamental transducer characteristics. The analysis begins by plotting $Z_{e e}$ on the complex plane as the reactive (imaginary) component versus the resistive (real) component. This complex plot, known as the Nyquist format, includes information arising 


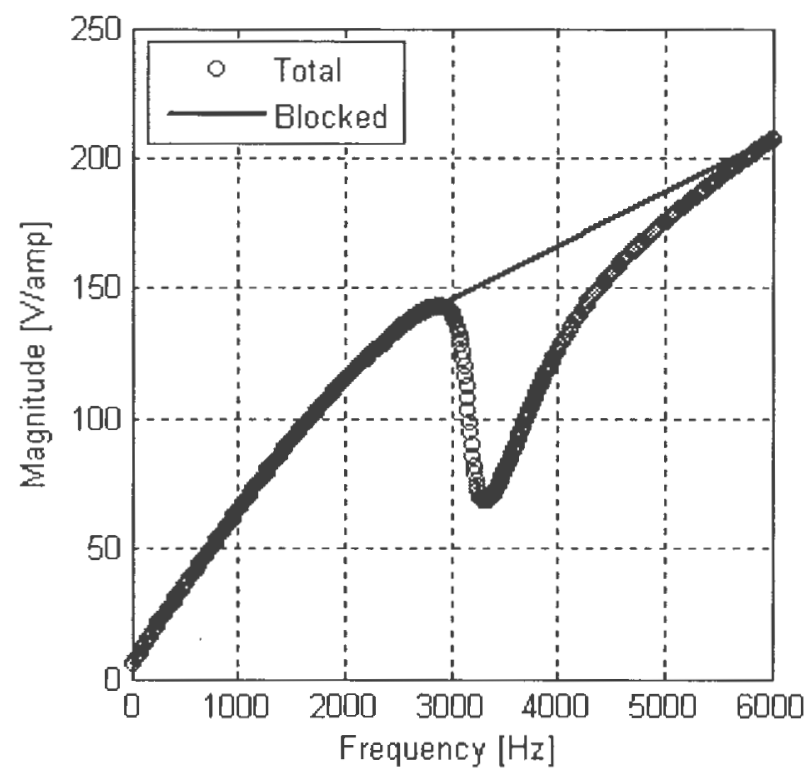

Figure 3.6: Experimental total electrical impedance and inferred blocked electrical impedance for a Terfenol-D vibratory motor from Etrema Products, Inc.

from both the magnitude and phase responses of the impedance. The impedance is in the frequency domain and as the frequency is changed, the tip of the impedance vector, which is the vector from the origin to the corresponding real and imaginary parts, traces a curve known as a locus. The locus in Figure 3.7 is the one traced by the electrical impedance vector measured from the Etrema Terfenol-D motor. The circle in the locus illustrates the effects of the motional component of the electrical impedance and is common to behavior at the mechanical resonance of an electromechanical transducer. Since the magnitude of the total electrical impedance does not contain phase information, the relative maximum of Figure 3.6 does not necessarily correspond to the resonant frequency. The resonance $f_{r}$ of the transducer is 


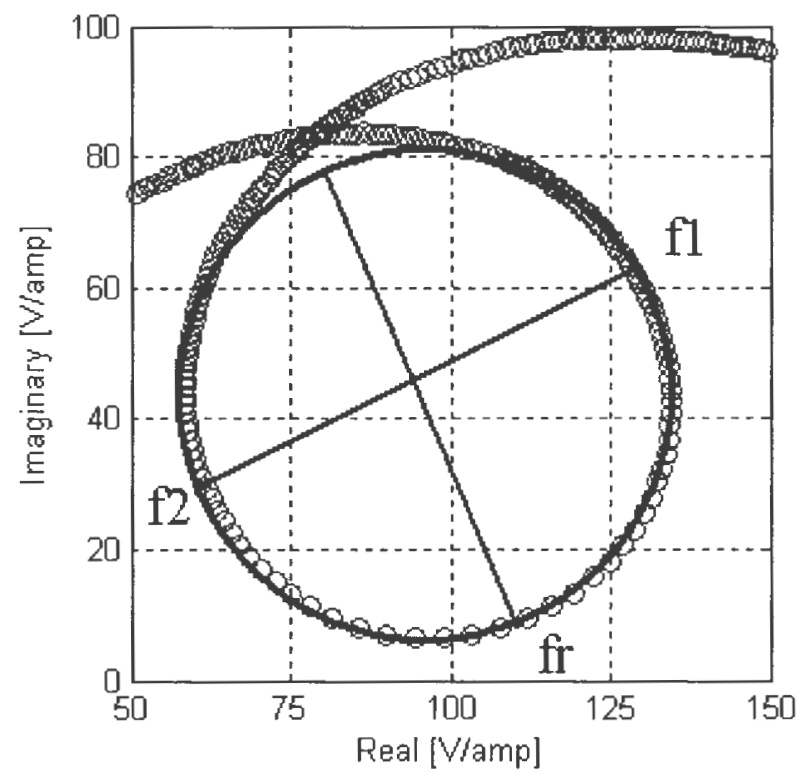

Figure 3.7: Nyquist plot of the experimental total electrical impedance due to the motional component for a Terfenol-D vibratory motor from Etrema Products, Inc.

determined by the principal diameter of the impedance mobility loop, that is the diameter that intersects the crossover point in the Nyquist plot of $Z_{e e}$. The two points at $90^{\circ}$ from the principal diameter are the half-power frequencies $f_{1}$ and $f_{2}$. The half-power frequencies occur where the magnitude of the response is 3- $\mathrm{dB}$ below the resonant peak.

An identical form of analysis is performed on the electrical admittance function and is defined as the inverse of the electrical impedance $Y_{e e}=1 / Z_{e e}$. The inverse relationship between electrical impedance and admittance means that this curve is simply the geometric reciprocal of the impedance locus with respect to unity. This does not mean that that identical information is presented in both. Although the total impedance and admittance are inverses of one another, the coupled motional 
effects are not i.e. $Y_{m o t} \neq 1 / Z_{\text {mot }}$. The electrical admittance is given in terms of the electrical impedance [17],

$$
Y_{e e}=\frac{I}{V}=Y_{e}+\frac{-\left(T_{e m} T_{m e} / Z_{e}^{2}\right)}{Z_{m}+Z_{L}+\left(T_{e m} T_{m e} / Z_{e}^{2}\right)}=Y_{e}+Y_{m o t}
$$

where $Y_{e}$ is the blocked electrical admittance. The circle create in the Nyquist diagram by the resonant motion of $Z_{e e}$ will have a corresponding circle in the admittance Nyquist diagram since the geometric inversion of a circle will still be a circle but with a different principal diameter. Figures 3.8-3.9 show the magnitude and Nyquist plot of the electrical admittance, respectively for the Etrema Terfenol-D tranducer. The blocked electrical admittance is the solid line in Figure 3.8; as in the electrical impedance, the motional component of the total electrical admittance is the deviation from this line. The principal diameter runs directly through the crossover point in the Nyquist plot and locates the antiresonance $f_{a r}$. Similarly, $90^{\circ}$ in either direction are the half-power frequencies $f_{a 1}$ and $f_{a 2}$ associated with the antiresonance.

An effective magnetomechanical coupling coefficient quantifying the conversion between magnetic and elastic energies in a magnetostrictive transducer can be calculated from the resonant and antiresonant frequencies, and is given by, [16]

$$
k_{e f f}^{2}=1-\left(\frac{f_{r}}{f_{a r}}\right)^{2} .
$$

This is a dynamic method of calculating the magnetomechanical coupling coefficient. An alternate method, the three parameter method, determines the material coupling coefficient $k$ through $k^{2}=q^{2} E_{y}^{H} / \mu^{\sigma}$, with $q$ the piezomagnetic coupling coefficient, $E_{y}^{H}$ the Young's modulus and $\mu^{\sigma}$ the magnetic permeability at constant stress. 


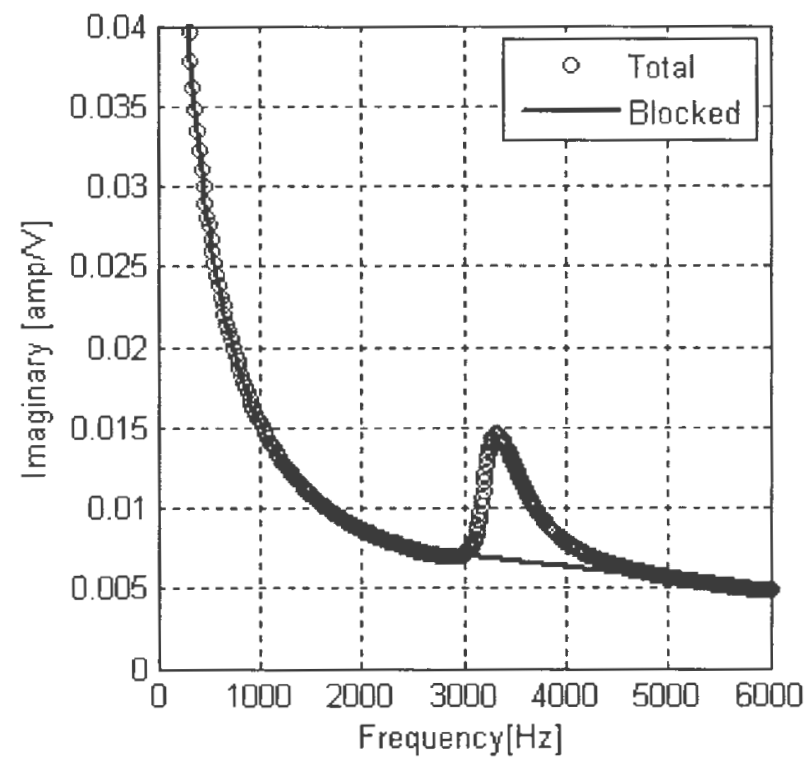

Figure 3.8: Electrical admittance and inferred blocked electrical admittance for a Terfenol-D vibratory motor from Etrema Products, Inc.

The resonant frequency is related to the effective mechanical stiffness $k_{m}$ through the relation,

$$
k_{m}=k_{t}+k_{d}+0.5 k_{f}=\left(2 \pi f_{r}\right)^{2}\left(\frac{m_{b} m_{e f f}}{m_{b}+m_{e f f}}\right)
$$

where $m_{b}$ is the mass of the transducer base and $m_{\text {eff }}$ is the effective mass of the Terfenol-D rod and piston [16]. Since the mass of the base is large enough to mechanically isolate the transducer dynamics from the test environment, it can be assumed to be infinite, i.e. completely rigid, resulting in a simplified expression for the mechanical stiffness of the Terfenol-D rod,

$$
k_{t}=\left(2 \pi f_{r}\right)^{2} m_{e f f}-k_{d}-0.5 k_{f} .
$$

The total stiffness of the transducer is a combination of the effective stiffness of the fluid, diaphragm and Terfenol-D rod. The stiffness of the diaphragm was measured 


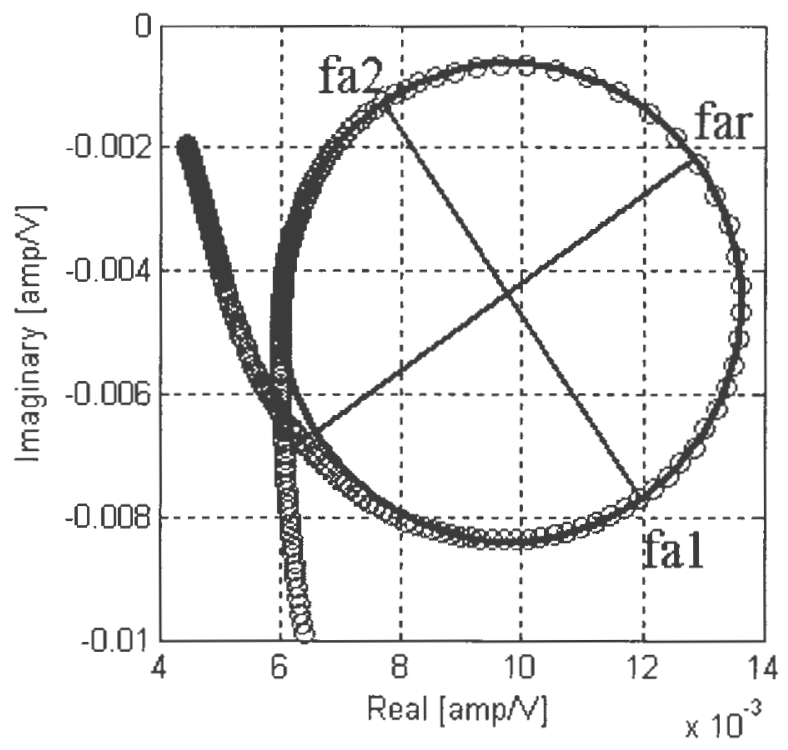

Figure 3.9: Nyquist plot of the experimental total electrical admittance for a TerfenolD vibratory motor from Etrema Products, Inc.

on a tabletop MTS compression machine while assembled in the transducer without the Terfenol-D rod and fluid and has value of $2.15 \mathrm{MN} / \mathrm{m}$. The Young's modulus of the Terfenol-D rod is then determined by combining equations (3.1) and (3.29). Once the stiffness and modulus of elasticity of the Terfenol-D rod are determined, the other coefficients in the Terfenol-D transducer model may be quantified. The undamped natural frequencies of the two degree of freedom model are determined by solving the eigenvalue equation $\operatorname{det}([\tilde{K}]-\lambda I)=0$, with the mass normalized stiffness matrix $[\tilde{K}]$ which is analogous to the single degree of freedom constant $\sqrt{\mathrm{k} / \mathrm{m}}$ and eigenvalues $\lambda=\omega^{2}[18]$. The natural frequency of the effective mass corresponds to the resonant frequency that determined $k_{m}$. The damping coefficients, $b_{i}$ and $b_{f}$, are subsequently 
determined by,

$$
\begin{aligned}
b_{f} & =2 \zeta_{f} \omega_{f} m_{f} \\
b_{i} & =2 \zeta_{t} \omega_{t} m_{e f f}-b_{f}
\end{aligned}
$$

where $\omega_{f}$ and $\omega_{t}$ are the undamped natural frequencies of the fluid and effective masses, respectively. The damping ratios are determined during model simulations but are typically expected to be less than 0.5 .

\subsubsection{Electroacoustics Equations}

The linear piezomagnetic constitutive relations (3.19)-(3.20) are coupled with electromechanical relations in order to derive expressions equivalent to equations (3.21)-(3.22) for the Terfenol-D transducer. The constitutive relations are manipulated into the form of the electroacoustic equations by utilizing electromechanical relations. It is assumed that the Terfenol-D rod completely fills an ideal wire solenoid of $N$ turns, length $L_{t}$ and cross-sectional area $A_{t}$ and that the current and magnetic field are linearly related [13]. The total flux through the $\operatorname{rod}$ is $\phi=B A_{t}$ and the flux linkage is $\lambda=N B A_{t}$. Assuming spatial independence of stress and strain, the strain in the Terfenol-D rod is related to the displacement $x$ by definition $\epsilon \equiv x / L_{t}$, and can be expressed in terms of velocity through its derivative. The stress on the rod is related to the force through the ratio of the cross-sectional area of the rod. The total voltage drop across the coil is the series combination of an electrical resistance and 
inductance. The electromechanical relations are given by,

$$
\begin{aligned}
H & =n I \\
\epsilon & =\frac{v}{j \omega L_{t}} \\
\sigma & =\frac{F}{A_{t}} \\
V & =R+j \omega N B A_{t},
\end{aligned}
$$

where $n=N / L_{t}$ is the turns ratio of the solenoid. By rewriting the constitutive equations for Terfenol-D using the electromechanical relations, the electroacoustic equations relating the input voltage and output force with respect to input current and output velocity for a Terfenol-D driven transducer are formed,

$$
\begin{aligned}
V & =\left[R+j \omega \mu^{\sigma}\left(1-k^{2}\right) n^{2} A_{t} L_{t}\right] I+N q k_{t} v \\
F & =-N q k_{t} I+\frac{k_{t}}{j \omega} v
\end{aligned}
$$

By comparison with the electroacoustic equations it is apparent that the blocked electrical impedance is the electrical series combination of an ideal resistor and inductor with the inductance $L$ expressed in terms of material properties,

$$
Z_{e}=R+j \omega \mu^{\sigma}\left(1-k^{2}\right) n^{2} A_{t} L_{t}=R+j \omega L_{b l o c k} .
$$

Electrical inductance for a general wire solenoid is defined as $\mu n^{2} A L$ [12]. The expression independently derived in equation (3.38) is related to the general definition by defining the permeability at constant strain as

$$
\mu^{\epsilon}=\mu^{\sigma}\left(1-k^{2}\right)
$$


The electromechanical transduction coefficients and blocked mechanical impedance are equivalently identified

$$
\begin{aligned}
T_{e m} & =N q k_{t} \\
T_{m e} & =-N q k_{t}=-T_{e m} \\
Z_{m} & =\frac{k_{t}}{j \omega}
\end{aligned}
$$

The transduction coefficients are equal in magnitude and opposite in direction and in accordance with principles for magnetostrictive transducers due to the spatial dependency on input current and magnetic field [17]. The blocked mechanical impedance contains only a stiffness term which is due to the constitutive equations ignoring the damping and inertia effects of Terfenol-D. This assumption made in the constitutive equations allows for high inaccuracies in the blocked mechanical impedance of Terfenol-D. Section 3.5.3 goes into greater detail about addressing the shortcomings of and alterations to the derived impedance expressions.

\subsubsection{Modified Blocked Impedances}

The expressions developed for the blocked mechanical and electrical impedances do not adequately describe the blocked mechanical and electrical behavior of the Terfenol-D transducer. Figure 3.10 shows the electrical drive point impedance measured from the Terfenol-D transducer and for the dynamic system model. The transducer shows a peak in the electrical impedance at approximately $9500 \mathrm{~Hz}$, while the dynamic system model continues to increase linearly. The presence of this peak illustrates a capacitive effect on the electrical drive point impedance. The coil in the Terfenol-D transducer is tightly wound insulated copper wire. The insulation is acting as a dielectric between windings which gives capacitive behavior to the coil. This 


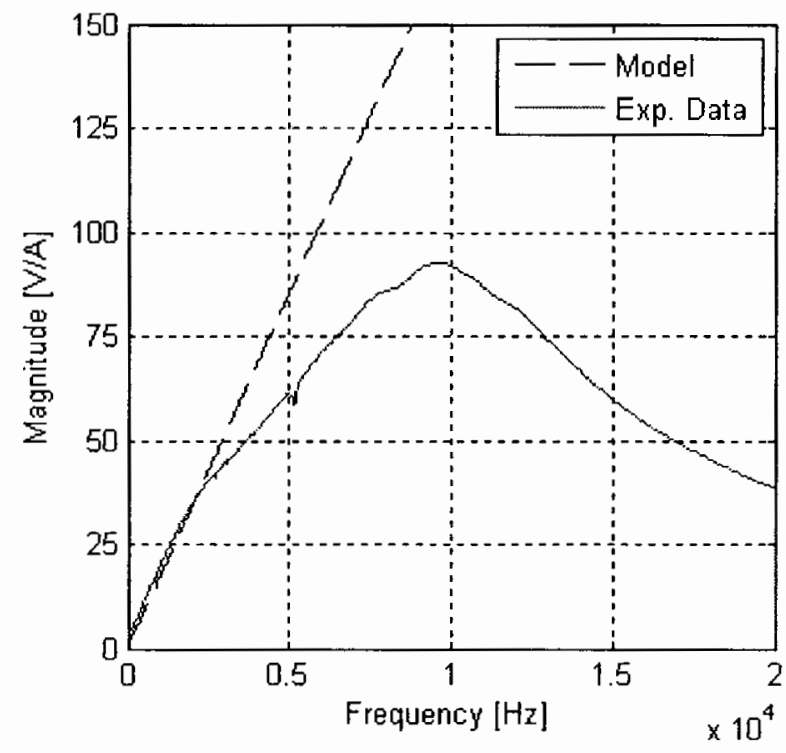

Figure 3.10: Electrical drive-point impedance for both the Terfenol-D pump and dynamic system model.

capacitance is in parallel with the coil resistance and inductance, as illustrated by the electrical circuit diagram in Figure 3.11. The modified blocked electrical impedance thus behaves like a bandpass filter

$$
Z_{e}=\frac{R+j \omega L_{b l o c k}}{L_{b l o c k} C(j \omega)^{2}+R C j \omega+1} .
$$

Figure 3.10 also shows the lack of motional effects of the model. The blocked mechanical expression found through the constitutive relations contains only a stiffness component and does not account for any inertia and damping effects of the Terfenol-D rod. The mechanical impedance of the transducer derived in equation (3.5) is a more complete expression for the blocked mechanical impedance as it describes a second order system that considers the inertia, damping and stiffness effects of the effective mass and is now used to describe the blocked mechanical impedance. 


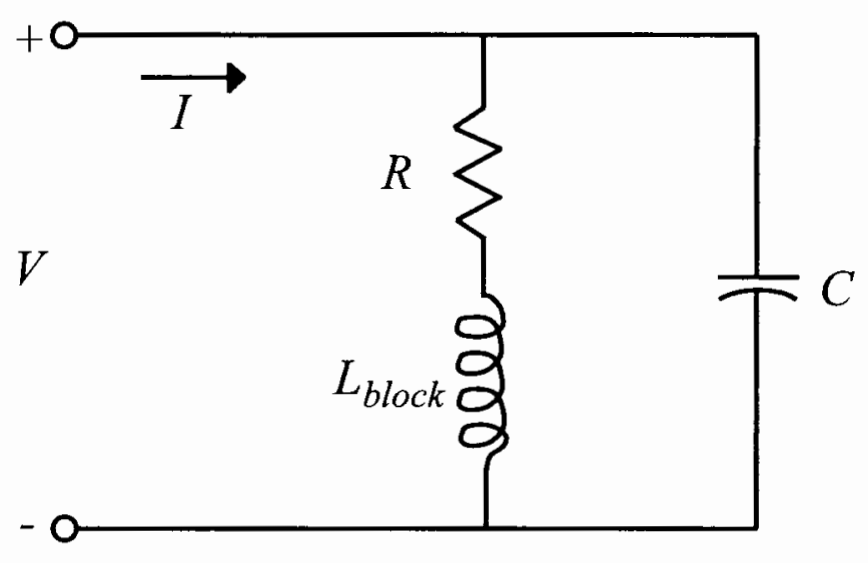

Figure 3.11: Circuit diagram showing the coil resistance and inductance in parallel to the capacitance due to the coil windings.

\subsubsection{Load Impedance}

In determining the electrical drive-point impedance $Z_{e e}$ of the Terfenol-D transducer under loaded conditions, a load impedance $Z_{L}$ due to the rest of the transducer is added to (3.25). The definition of the load impedance, equation (3.23), provides a method for developing an expression for $Z_{L}$ by relating it to the resultant force $F$ on the transducer. Force $F$ is defined in (3.22) as the superposition of a term proportional to the applied current $I$ and the product of the blocked mechanical impedance, defined in (3.5), and the velocity $v$ of the Terfenol-D transducer. Equations of motion for the vibratory model of the Terfenol-D transducer, (3.2)-(3.3), rewritten by noting that $\alpha=-T_{m e}$ and $v=\dot{x}_{t}$, to obtain an equivalent expression for the resultant force $F$. This expression for the resultant force is utilized with the definition of the load 
impedance to yield an expression for $Z_{L}$ in the frequency domain,

$$
\begin{aligned}
F & =T_{m e} I+Z_{m} v=-Z_{L} v \\
& =\frac{1}{2}\left[-\left(b_{f} j \omega+k_{f}\right) X_{t}+\left(b_{f} j \omega+k_{f}\right) X_{f}\right] \\
Z_{L} & =\frac{\left(b_{f} j \omega+k_{f}\right)-\left(b_{f} j \omega+k_{f}\right) \frac{X_{f}}{X_{t}}}{2 j \omega} .
\end{aligned}
$$

The load impedance is dependent on the ratio of fluid displacement to effective mass displacement. A formula is developed to express this ratio as a frequency response function dependent on the input current $I$ and pumping chamber pressure $P_{1}$. Equations (3.2)-(3.3) are represented in the frequency domain as a matrix equation,

$$
\left[\begin{array}{cc}
m_{e f f}(j \omega)^{2}+\left(b_{i}+\frac{b_{f}}{2}\right) j \omega+k_{i}+\frac{k_{f}}{2} & -\frac{1}{2}\left(b_{f} j \omega+k_{f}\right) \\
-\frac{1}{2}\left(b_{f} j \omega+k_{f}\right) & m_{f}(j \omega)^{2}+b_{f} j \omega+k_{f}
\end{array}\right]\left[\begin{array}{c}
X_{t} \\
X_{f}
\end{array}\right]=\left[\begin{array}{l}
-T_{m e} I \\
-P_{1} A_{i}
\end{array}\right]
$$

which is solved using Cramer's rule. The deflection of the Terfenol-D rod thus takes the form,

$$
X_{t}=\frac{\operatorname{det}\left(\begin{array}{cc}
-T_{m e} I & -\frac{1}{2}\left(b_{f} j \omega+k_{f}\right) \\
-P_{1} A_{i} & m_{f}(j \omega)^{2}+b_{f} j \omega+k_{f}
\end{array}\right)}{\operatorname{det}\left(\begin{array}{cc}
m_{e f f}(j \omega)^{2}+\left(b_{i}+\frac{1}{2} b_{f}\right) j \omega+k_{i}+\frac{1}{2} k_{f} & -\frac{1}{2}\left(b_{f} j \omega+k_{f}\right) \\
-\frac{1}{2}\left(b_{f} j \omega+k_{f}\right) & m_{f}(j \omega)^{2}+b_{f} j \omega+k_{f}
\end{array}\right)} .
$$

The displacement of the fluid mass is solved similarly. The overall expression for the displacement ratio frequency response function, written in terms of the input current and outp1t pressure is given by the formula,

$$
\frac{X_{f}}{X_{t}}=-\frac{\frac{T_{m e}}{2}\left(b_{f} j \omega+k_{f}\right)+A_{i}\left[m_{e f f}(j \omega)^{2}+\left(b_{i}+\frac{1}{2} b_{f}\right) j \omega+k_{i}+\frac{1}{2} k_{f}\right] \frac{P_{1}}{I}}{T_{m e}\left[m_{f}(j \omega)^{2}+b_{f} j \omega+k_{f}\right]+\frac{A_{i}}{2}\left(b_{f} j \omega+k_{f}\right) \frac{P_{1}}{I}} .
$$

The inclusion of the load impedance concludes the required derivations of all aspects of the dynamic system model. 


\subsection{Full System Model and Simulations}

Combining the modified expressions for blocked electrical and mechanical impedances, the transduction coefficients and the load impedance into equation (3.25), the electrical drive point impedance is developed for the Terfenol-D transducer,

$$
\begin{aligned}
Z_{e e}= & \frac{R+j \omega L_{\text {block }}}{L_{\text {block }} C(j \omega)^{2}+R C j \omega+1} \\
& +\frac{\left(N q k_{t}\right)^{2} j \omega}{m_{e f f}(j \omega)^{2}+\left(b_{i}+\frac{1}{2} b_{f}\right) j \omega+k_{i}+\frac{1}{2} k_{f}-\frac{1}{2}\left(b_{f} j \omega+k_{f}\right) \frac{X_{f}}{X_{t}}} .
\end{aligned}
$$

where $X_{f} / X_{t}$ couples the total electrical impedance frequency response function with the mechanical and fluid state space model through the chamber pressure $P_{\mathbf{1}}$ and input current $I$. Since the state space portion of the model is solved in the time domain, a stepped sine simulation is written in which the state space system is solved for each frequency and concurrently inputs the corresponding chamber pressure and input current into the total electrical impedance.

Determining the various parameters is critical for proper model simulations. Some parameters are predetermined or can be easily measured or calculated such as masses of individual components, diaphragm stiffness, fluid line resistance and fluid capacitance of the pumping chamber, accumulator and hydraulic cylinder. The parameters whose variances cause the largest fluctuations are those dealing with the magnetostrictive motion of Terfenol-D (i.e. the piezomagnetic coupling coefficient $q$, the Young's modulus $E_{y}^{H}$, the magnetic permeability at constant stress $\mu^{\sigma}$, the magnetoelastic coupling coefficient $k$ ), the fluid parameters (fluid viscosity, density and bulk modulus) and damping ratios. The Young's modulus is perhaps the most important parameter in that it controls the location of the transducer resonance as given by equation (3.29). Parameters $q, \mu^{\sigma}$ and $k$ are all related to $E_{y}^{H}$ through the 
three parameter method while the magnetoelastic coupling coefficient is estimated experimentally through equation (3.27). The fluid parameters are provided by the manufacturer and depending on the amount of entrained air trapped in the fluid, the bulk modulus may vary greatly. Methods for removing entrained air are described in Section 4.2.1. The damping ratios for the two degree of freedom model describing the magnetostrictive pump greatly affect the magnitude and curvature of the motional impedance $Z_{m o t}$. There is no good measure for damping in the pump but with an understanding of its influence on the motional impedance, the damping ratios are identified by fitting the magnitude of the motional impedance to experimental motional impedance data.

The blocked electrical impedance accounts for the capacitive effects due to the Terfenol-D transducer drive coil. The capacitance is determined by the location of the coil resonance. From analyzing dynamic tests, the peak in the total electrical impedance occurs at approximately $9500 \mathrm{~Hz}$. This resonance is related to the coil inductance and capacitance. For a given inductance, the capacitance is found through the relation,

$$
\omega_{\text {coil }}=\frac{1}{\sqrt{L_{\text {block }} C}}
$$

Figure 3.12 illustrates the electrical resonance of the blocked electrical impedance. Clearly there is not enough damping in the modeled expression for $Z_{e}$ as the model gives a peak that is 40 times larger than the dynamic data. In the expression for the blocked electrical impedance, the resistance is a measure of the damping in the system. It is also noted that the coil has a DC resistance of $2 \Omega$. In order to increase the electrical damping but also maintain a DC resistance of $2 \Omega, Z_{e}$ is modified to 


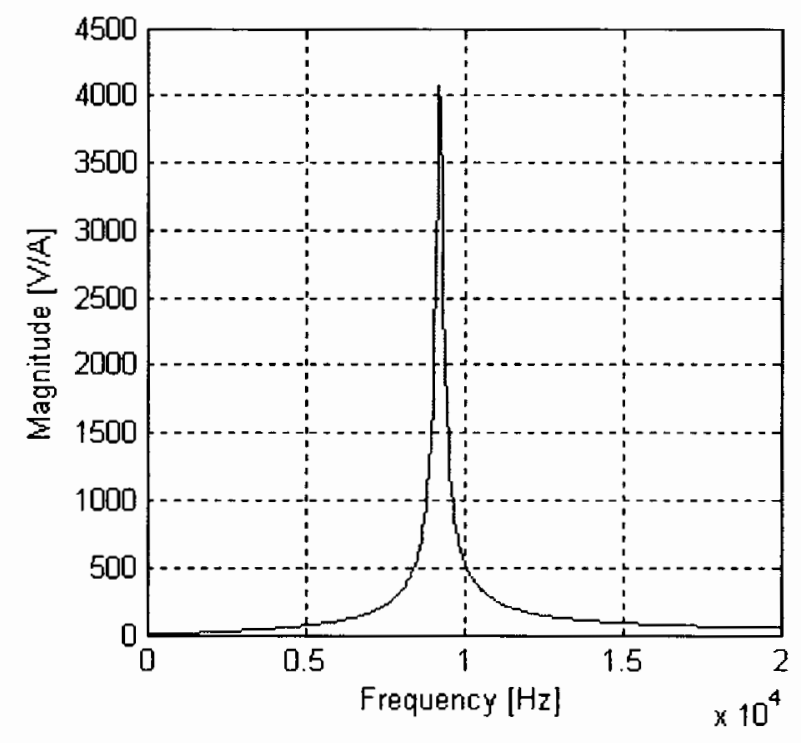

Figure 3.12: Model simulation of the blocked electrical impedance a low little damping value of 0.013 .

incorporate both effects,

$$
Z_{e}=\frac{R+j \omega L_{\text {block }}}{L_{\text {block }} C(j \omega)^{2}+\left(R C+\alpha_{K V}\right) j \omega+1}
$$

where $R$ remains the DC resistance of the drive coil and $\alpha_{K V}$ is a Kelvin-Voight correction factor that accounts for any damping overlooked by the model. Figure 3.13 shows the model simulation of the Terfenol-D transducer alongside data obtained from harmonic and dynamic testing. There is good correlation between the model and test data. A more detailed discussion is given in Chapter 5, while Tables A.1-A.2 in Appendix A list the values for the mechanical and fluid parameters of the Terfenol-D transducer used in the dynamic system model. 


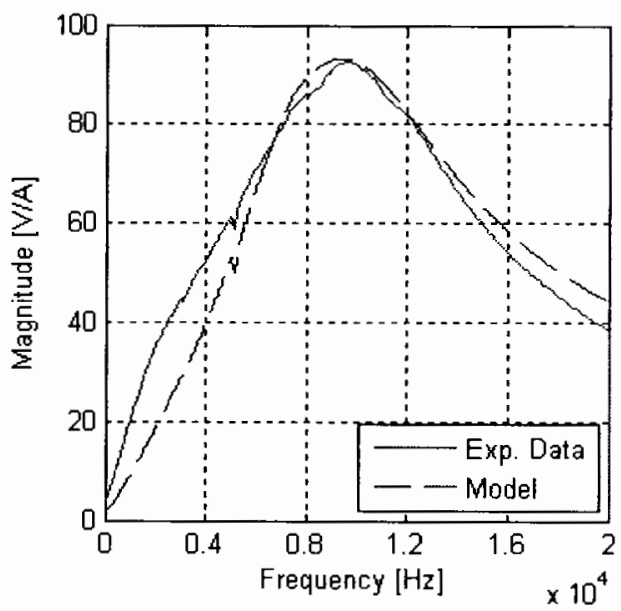

(a)

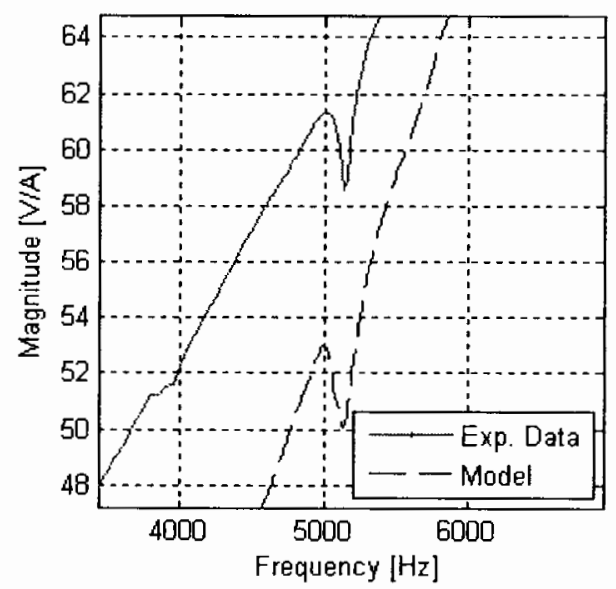

(c)

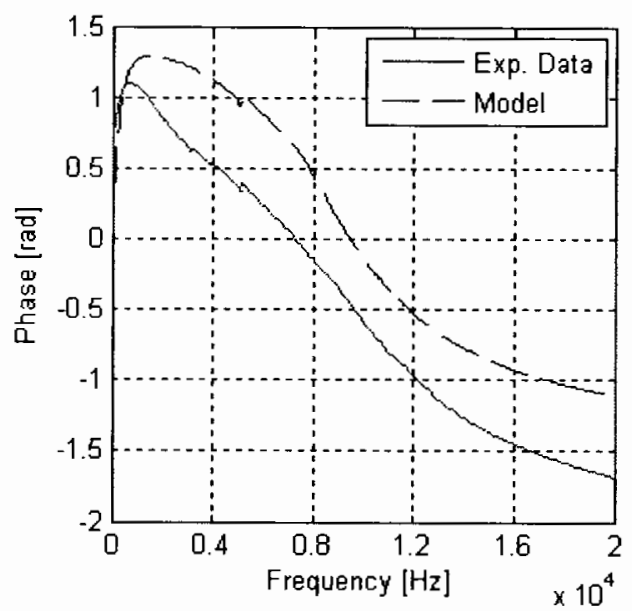

(b)

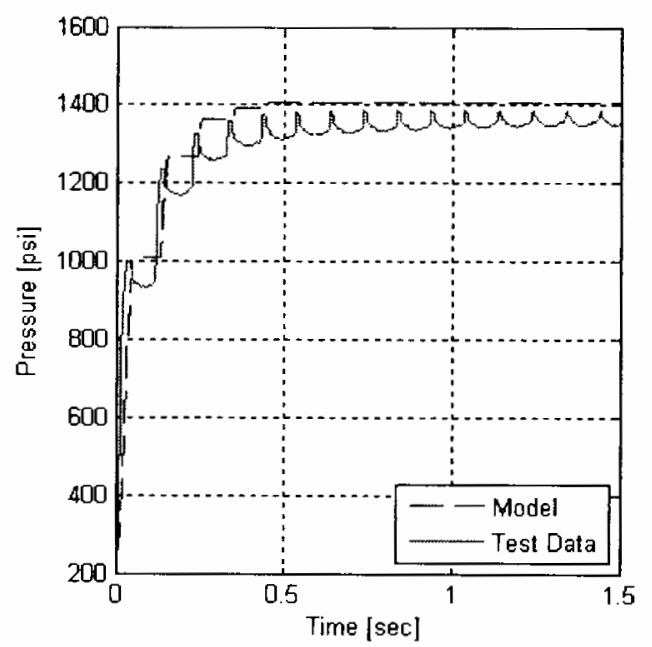

(d)

Figure 3.13: Model simulation and experimental test data: (a) Magnitude response of $Z_{e e}$, (b) Phase response of $Z_{e e}$, (c) Zoomed magnitude response of $Z_{e e}$ and (c) Maximum pressure time trace under blocked output conditions $(10 \mathrm{~Hz})$. 


\section{CHAPTER 4}

\section{TERFENOL-D PUMP DESIGN AND TESTING}

This chapter presents the design and testing of the Terfenol-D pump. The points of focus for the transducer design are the mechanical components, magnetic circuit and hydraulic interface. Harmonic and broadband testing is performed on the Terfenol-D pump with the goal to quantify the maximum output pressure differential, maximum no-load velocity, the frequency limitation of the reed valves and input electrical impedance of the transducer.

\subsection{Mechanical Design}

The design of the Terfenol-D pump is centered around the safe and optimal performance of the Terfenol-D rod. Figures 4.1-4.2 show exploded cutaway views of the solid model of the pump. As discussed in Chapter 2, operating the Terfenol-D rod under compression serves two purposes: (i) aligns the magnetic moments perpendicular to the axial direction of the rod and (ii) protects the rod by preventing operation in tension. The transducer includes a preloading mechanism consisting of a seat, transducer base, adjustor cap and preload bolt (not shown). The bolt threads into the adjustor cap and applies a compressive stress to the Terfenol-D rod through the seat. The rod is restrained at its opposite end by the piston, diaphragm and 


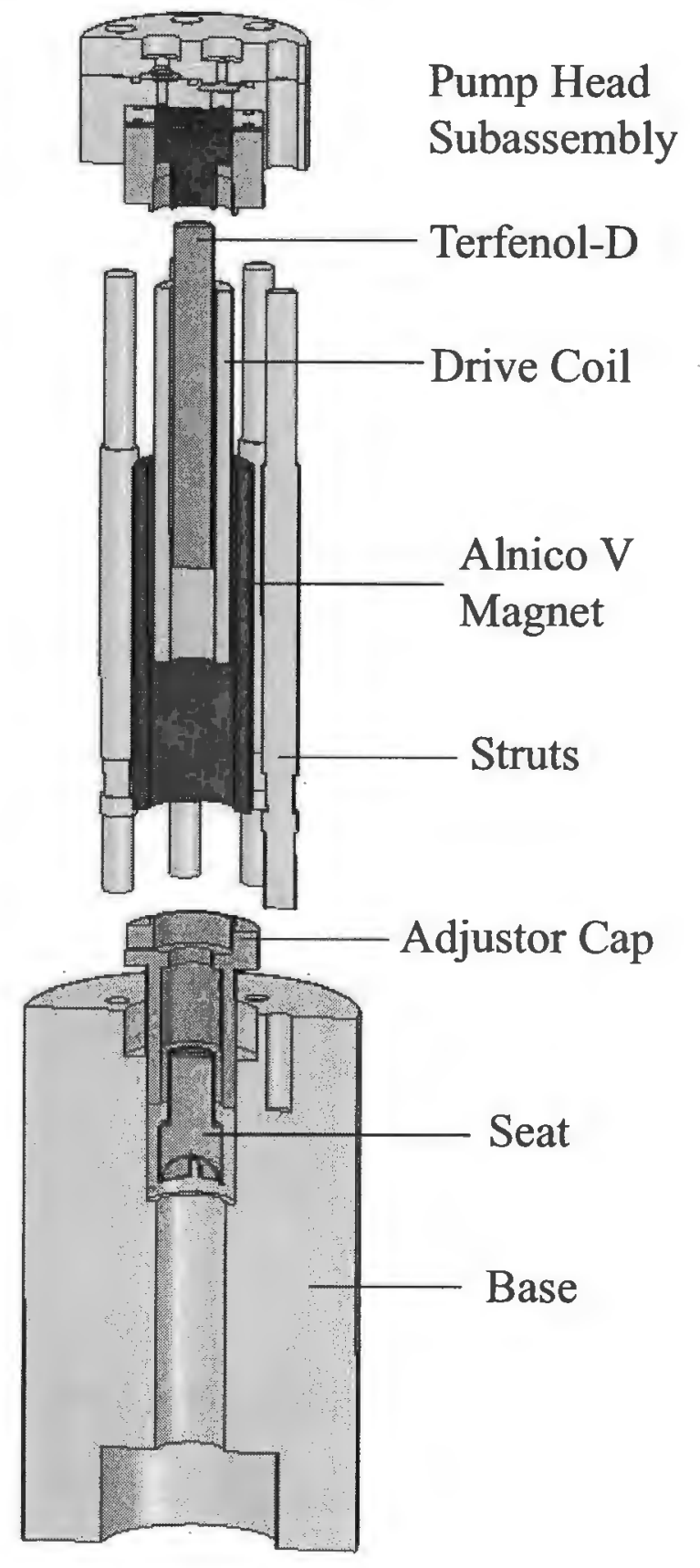

Figure 4.1: Exploded solid model of the Terfenol-D pump. 


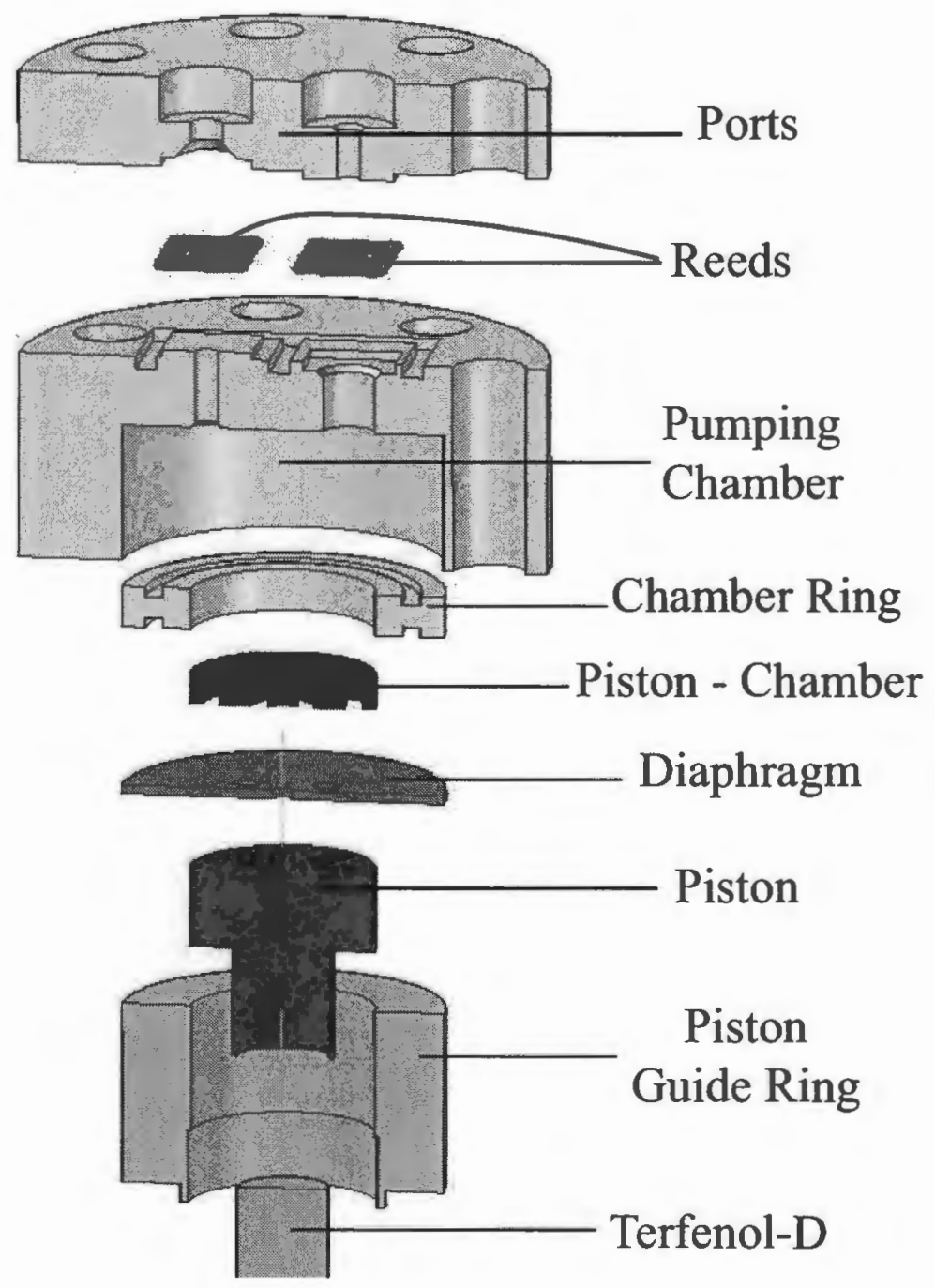

Figure 4.2: Exploded solid model of the Terfenol-D pump head subassembly.

hydraulic fluid in the pumping chamber. The method for determining the preload on the rod is discussed in Section 4.2.2.

The piston subassembly consists of three parts, two piston pieces and the 0.005 in stainless steel diaphragm. The two piston components clamp the middle of the diaphragm. When the pump head is fully assembled, the outer edge of the diaphragm 
is clamped between the chamber ring and piston guide ring. The piston subassembly components move together during pump operation. The chamber ring and piston guide ring also provide a sliding fit with the piston. The alignment between the pump head subassembly and transducer base is critical in that any misalignment may cause damage to the Terfenol-D rod. Six Alnico $V$ magnets $(1.5$ in OD $\times 1.125$ in ID $\times 0.75$ in long) are stacked which provide the alignment between the pump head subassembly and transducer base and also provide a bias magnetic field through the Terfenol-D. The magnets are cast, have a tolerance on all dimensions of $+/-0.016$ in and require machining in order to provide proper alignment. Machining guidelines and assembly directions for magnet alignment are given in Appendix B.1.

The diaphragm is a critical component of the transducer. The preliminary design described in Appendix C.1 utilizes a dynamic O-ring for sealing purposes which is a simpler method of sealing but inhibits the performance of the pump due to excessive compliance in the pumping chamber. Any additional compliance in the pumping chamber results in efficiency losses. Although a method for quantifying the total compliant losses due to the O-ring is not readily available, assumptions are made by analyzing and comparing compression tests performed on the O-ring and diaphragm. Compression tests are performed on a tabletop MTS compression system by recording the displacement and force in the direction of piston motion. Figure 4.3 illustrates the results for both the Parker \#2-228 O-ring and 0.005 in diaphragm. The measured stiffness of the O-ring and diaphragem are $0.603 \mathrm{MN} / \mathrm{m}$ and $2.19 \mathrm{MN} / \mathrm{m}$, respectively. Although the diaphragm is approximately three times stiffer than the O-ring in the axial direction of transducer motion, the compression losses of the O-ring will have a greater affect during pump operation. As pressure is created inside the chamber, 


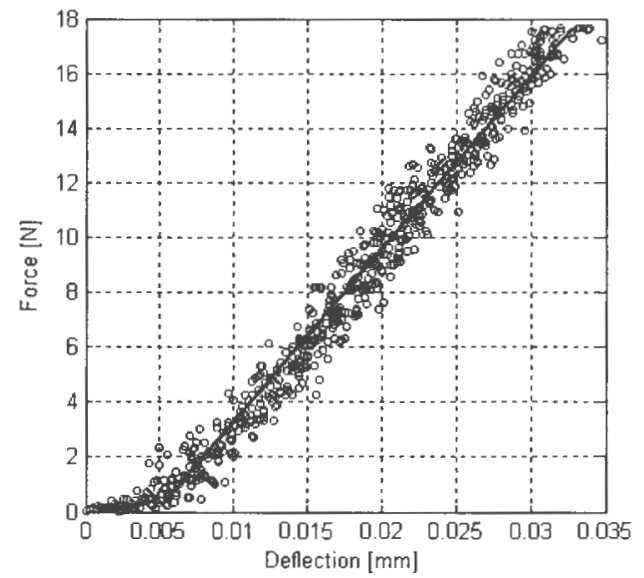

(a)

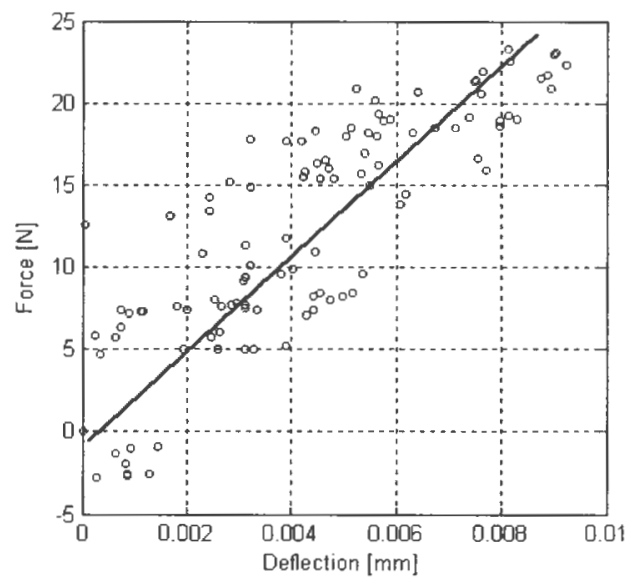

(b)

Figure 4.3: Compression tests for (a) Parker \#2-228 O-ring and (b) 0.005 in diaphragm.

it expands in all directions and since the O-ring has significant amount surface area on its ID exposed to the chamber, the pressure will compress the O-ring in the radial direction. Any energy wasted on compressing the O-ring causes a decrease in the volumetric efficiency and subsequently, on the maximum attainable pressure of the pump. By utilizing a stainless steel diaphragm seal, these compliant losses are effectively eliminated. The diaphragm has another function in that it acts as a spring for the preload mechanism.

The two components making up the pump head provide a way for clamping the reeds in place. There are both a recess and protrusion (reed valve seat) on both parts which when assembled hold the reed in place. Figure 4.4(a) shows one of the reed valves used in the pump. The outer part of the reed (everything excluding the flap) is completely fixed when assembled. The reed valve seats and reed valves are polished using a 6 micron diamond paste to improve to the metal to metal sealing and reduce 


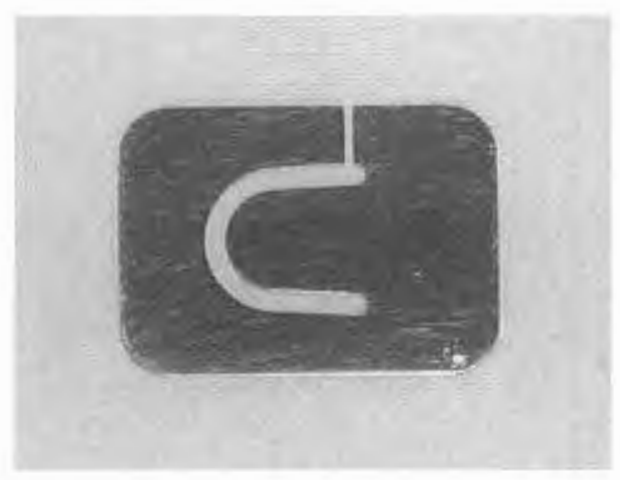

(a)

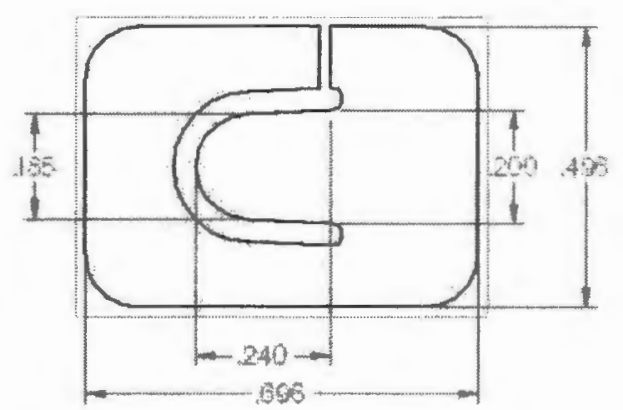

(b)

Figure 4.4: (a) Picture of reed valve used in Terfenol-D transducer and (b) planar dimensions of reed valve.

leakage losses. The pump head is designed with a figure eight groove that provides additional sealing in between the two pump head components. The chamber height is controlled by the thickness of the chamber ring, which allows to adjust the distance between the top of the piston and pump head (0.050 in). Pictures of the Terfenol-D transducer and of selected parts and subassemblies are given in Figures 4.5-4.6

\subsubsection{Reed Design}

One of the major aspects of the transducer design is the implementation of mechanical reed (check) valves for frequency rectification of the oscillatory pump flow creating a high-pressure unidirectional flow. The previous pump design, presented in Appendix C, was a two chamber design with oscillatory flow in which the fluid would need to be rectified by an external valve system. Since the magnitude of the magnetostrictive vibrations are on the order of thousandths of an inch, any losses between the pump chamber and the external valves would severely affect the performance of 


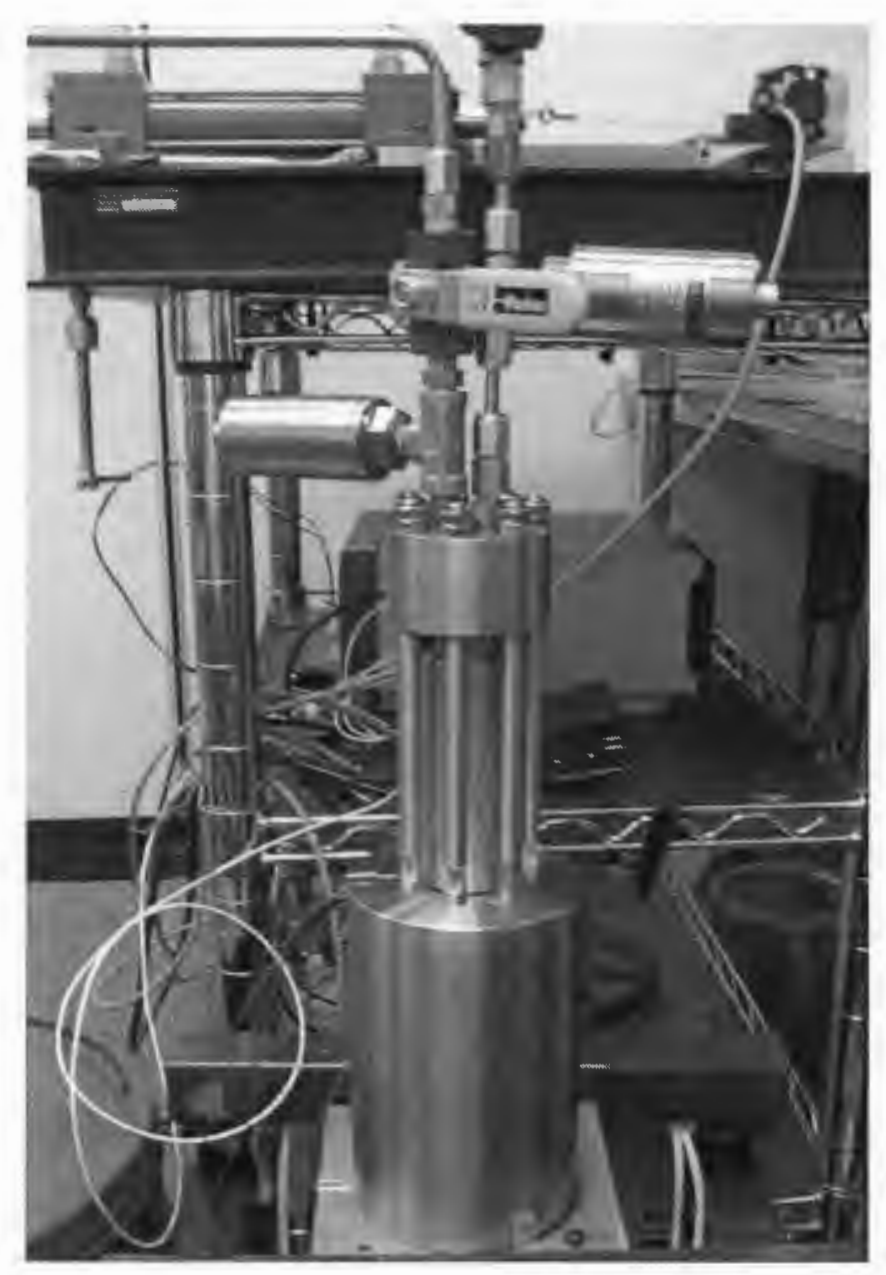

Figure 4.5: Picture of the Terfenol-D pump.

the pump. Rectification is performed at the pump head by mechanical reed valves in an attempt to eliminate these losses.

The reed valves are designed with a much larger natural frequency in air than at which the reeds are intended to operate. The presence of the hydraulic fluid has shear loading effects on the reeds which significantly reduce the maximum working frequency. The keys to designing the reed valves are making the reeds as stiff as possible with as little mass as possible but with enough surface area to seal fluid port 


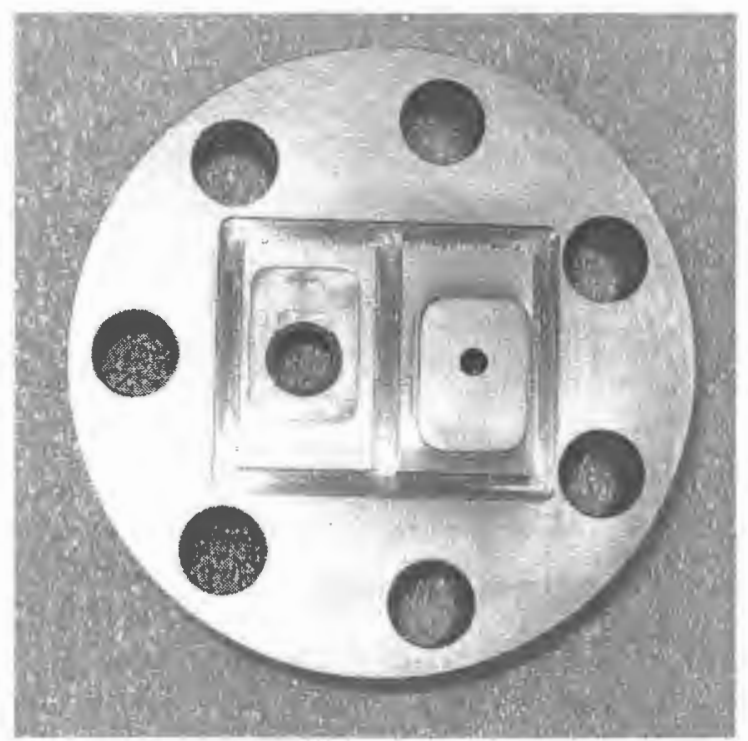

(a)

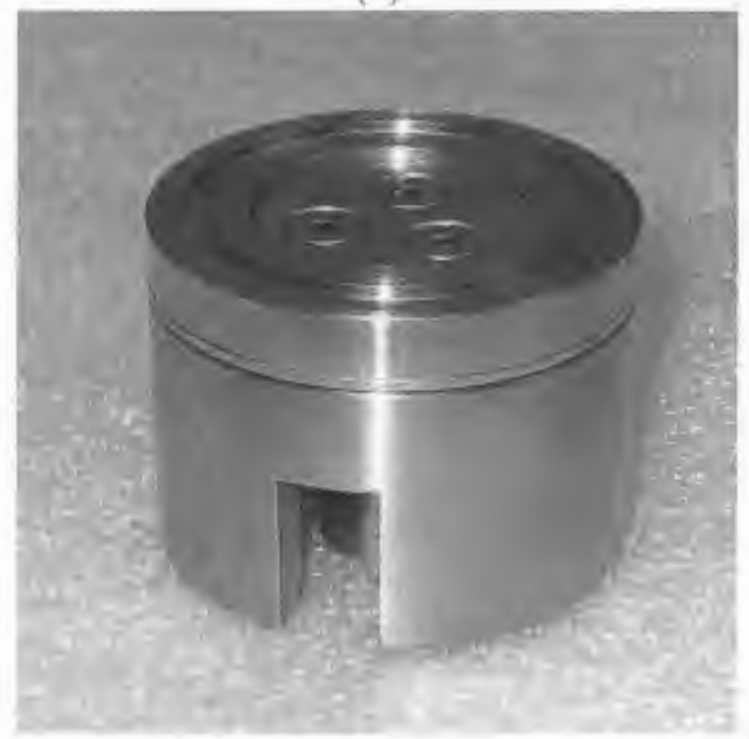

(c)

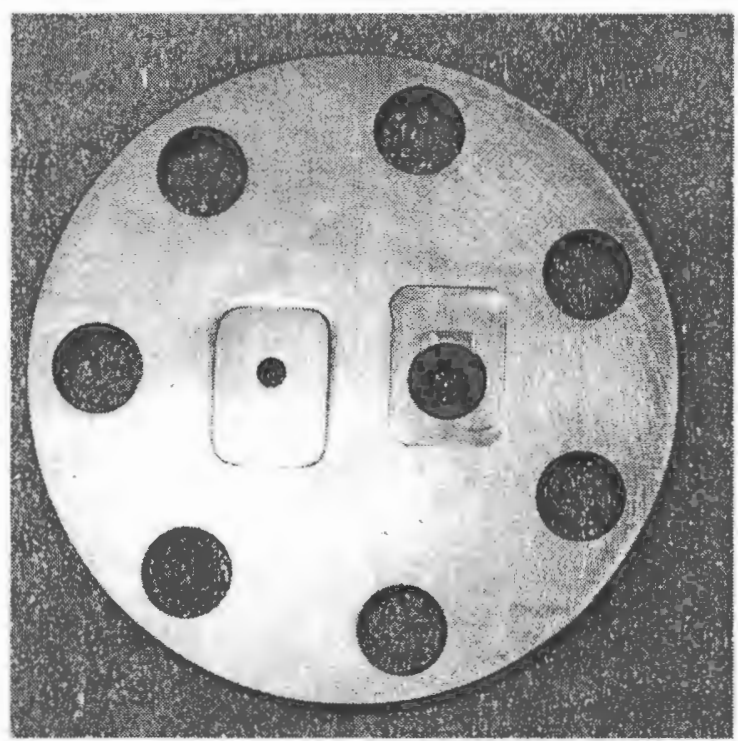

(b)

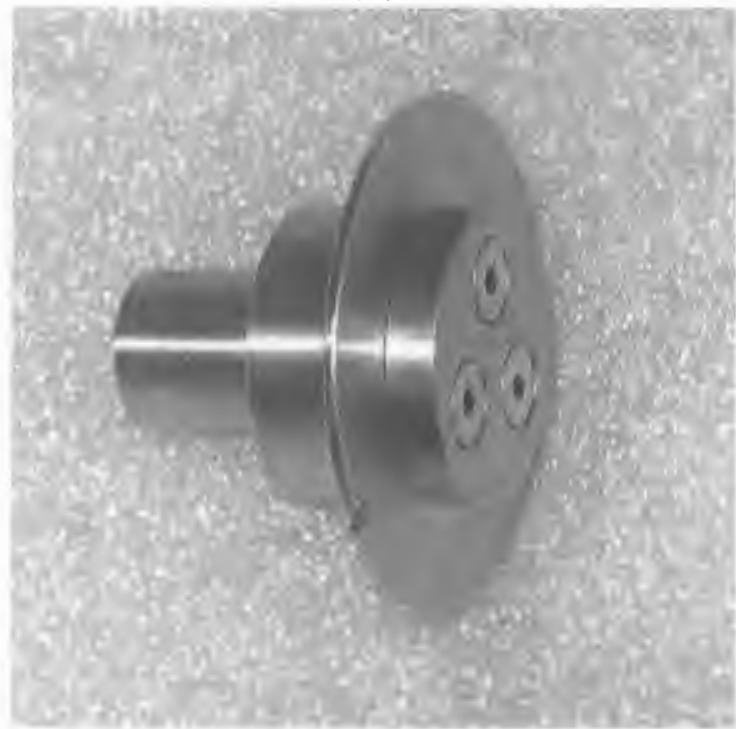

(d)

Figure 4.6: Pictures of the reed seats in the pump head (a) chamber housing and (b) port housing, (c) piston subassembly including the chamber and piston guide rings and (d) piston and diaphragm subassembly.

diameters of reasonable size. Steel tubing with an ID of $1 / 8$ in and OD of $1 / 4$ in is used to connect the pump to the actuator, thus the fluid ports will have the same ID 


\begin{tabular}{|c|c|c|}
\hline Type of Steel & SS (Type 301-FH) & 1095 BT\&P Spring \\
\hline$\rho_{s}\left[\mathbf{l b} / \mathbf{i n}^{3}\right]$ & 0.290 & 0.284 \\
\hline$E[\mathbf{k s i}]$ & 28,400 & 29,700 \\
\hline$S_{y}[\mathbf{k s i}]$ & 140 & 116 \\
\hline$S_{u t}[\mathbf{k s i}]$ & 185 & 184 \\
\hline
\end{tabular}

Table 4.1: Material properties for materials being considered for the reed valves: type 301 full hardened stainless steel and 1095 blue tempered and polished spring steel. The material properties given are material density $\rho_{s}$, modulus of elasticity $E$, yield strength $S_{y}$ and ultimate tensile strength $S_{u t}$.

in order to create a uniform flow path to and from the pumping chamber. To ensure that there is enough surface area to effectively cover the flow orifice, the tip of the reed flap has 0.030 in of excess radial length over the flow orifices. This is the minimum amount of material allowed for proper sealing of the reed valves. The reed also must not be too stiff which would result in insufficient reed tip deflection and thus increase the resistance losses across the reed valve. The chosen planar reed valve geometry which allows for adequate reed valve deflection is illustrated in Figure 4.4(b). The reeds are machined using a wire EDM.

The material properties for metals under consideration for the reed valves are given in Table 4.1. The frequency limit of the reed valve is assumed to be its natural frequency. The following calculations assume that the reed flap acts as a cantilever beam with a total length equal to that of the reed and a width equal to that of the base of the reed. For a cantilever beam with with length $l$, width $b$, and thickness $t$, the natural frequency is defined as [21]

$$
\omega_{n}^{2}=3.52^{2} \frac{E I}{m l^{3}}
$$




\begin{tabular}{|c||c|c|c|}
\hline \multirow{2}{*}{ Material } & Thickness & \multicolumn{2}{|c|}{ Natural Frequency } \\
& {$[\mathrm{in}]$} & MATLAB [Hz] & FEMLAB [Hz] \\
\hline \hline \multirow{3}{*}{ SS 301-FH } & 0.005 & 2727 & 3030 \\
\cline { 2 - 4 } & 0.010 & 5455 & 6059 \\
\cline { 2 - 4 } & 0.015 & 8182 & 9076 \\
\hline \multirow{3}{*}{ 1095 BT\&P } & 0.005 & 2821 & 3134 \\
\cline { 2 - 4 } & 0.010 & 5642 & 6267 \\
\cline { 2 - 4 } & 0.015 & 8464 & 9388 \\
\hline
\end{tabular}

Table 4.2: Natural frequencies of reed valve flap for thickness $0.005 \mathrm{in}, 0.010 \mathrm{in}$ and 0.015 in.

where $E$ is the modulus of elasticity, $m$ is the mass, and $I$ is the second moment of inertia defined as $I=b t^{3} / 12$. An m-file was created in MATLAB using equation (4.1) which provides quick approximations of the natural frequencies of a cantilever beam in air.

Since the the reed valves being considered have a slightly different geometry than a rectangular cantilever beam, a more exact method of determining the natural frequency is required. FEMLAB 3 Multiphysics Modeling by Comsol is used to more accurately approximate the natural frequencies for the exact reed geometry. Three thicknesses are investigated for type 301 full hardened stainless steel and 1095 blue tempered and polished spring steel. Table 4.2 compares the natural frequencies obtained from MATLAB and FEMLAB for the reed planar geometry described in Figure 4.4(b) with thicknesses 0.005 in, 0.010 in and 0.015 in. FEMLAB gives slightly larger frequencies for each thickness which is expected due to the fact that the reed geometry in FEMLAB has less mass than the cantilever beam with the same length and base width. A FEMLAB model of one of the reeds is shown in Figure 4.7. 


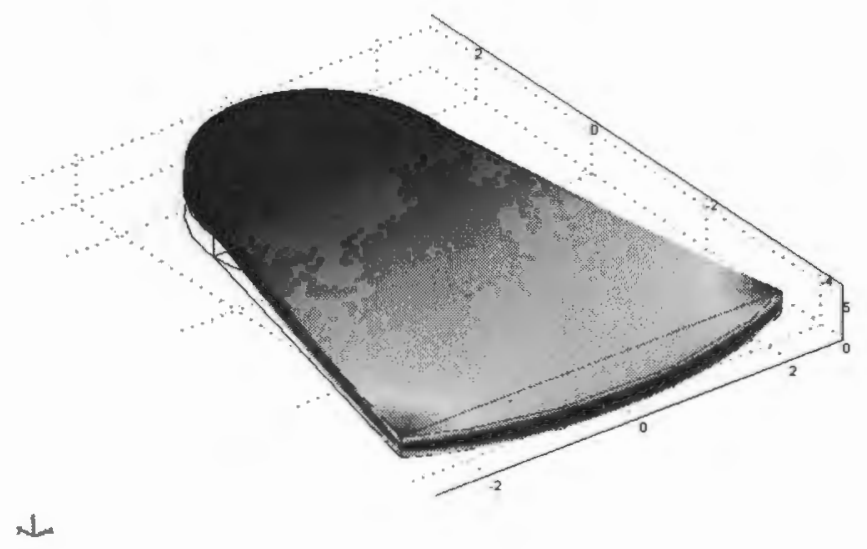

Figure 4.7: FEMLAB model reed flap.

One of the most important design considerations of the reed is infinite fatigue under the maximum applied stress on the reed. The following set of fatigue calculations refer to [21]. Fatigue calculations begin with determining the endurance limit of the material. The uncorrected endurance limit for steels with an ultimate tensile strength less than $200 \mathrm{ksi}$ is one half the ultimate tensile strength. In order to find the maximum allowable alternating stress $\sigma_{a}$ that may be experienced by the reed valves and still maintain infinite life, the modified Goodman failure criterion is used,

$$
\sigma_{a}=\frac{S_{e}}{1+\frac{S_{e}}{S_{u t}}}
$$

where $S_{e}$ is the corrected endurance limit (43.3 ksi [21]) for both materials and $S_{u t}$ is the ultimate tensile strength given in Table 4.1. This calculation resulted in a maximum alternating stress of $70 \mathrm{ksi}$ for both materials under consideration. The maximum stress that the reed undergoes is the sum of the alternating stress and the mean stress, which in this case is $\sigma_{\max }=2 \sigma_{a}=140 \mathrm{ksi}$. This is the maximum stress that the reed can withstand without enduring critical fatigue. The deflection 


\begin{tabular}{|c||c|c|}
\hline Material & $\begin{array}{c}\text { Thickness } \\
{[\mathrm{in}]}\end{array}$ & $\begin{array}{c}\text { Maximum Tip Deflection } \\
{[\mathrm{in}]}\end{array}$ \\
\hline \hline \multirow{3}{*}{ SS 301-FH } & 0.005 & 0.0189 \\
\cline { 2 - 3 } & 0.010 & 0.00946 \\
\cline { 2 - 3 } & 0.015 & 0.00631 \\
\hline \multirow{3}{*}{ 1095 BT\&P } & 0.005 & 0.0181 \\
\cline { 2 - 3 } & 0.010 & 0.00905 \\
\cline { 2 - 3 } & 0.015 & 0.00603 \\
\hline
\end{tabular}

Table 4.3: Maximum deflection of the reed flap tip for thicknesses of 0.005 in, 0.010 in and 0.015 in.

of the tip of the reed under these maximum stress conditions is now calculated. The deflection of the tip of the reed under an applied force $F$ is given by

$$
y_{\max }=\frac{F L^{3}}{3 E I}
$$

in which $F$ is related to the maximum stress through

$$
\sigma_{\max }=\frac{F L t}{2 I} .
$$

The maximum tip deflection for a cantilever beam under maximum stress conditions is found by solving (4.4) for $F L / I$ and substituting into (4.3) to get,

$$
y_{\max }=\frac{2 L^{2} \sigma_{\max }}{3 t E} .
$$

Table 4.3 shows the maximum tip deflection for each thickness.

Although carbon 1095 BT\&P spring steel gives the largest natural frequency, it is highly magnetic and it is not known if magnetization in the reeds will affect the pump operation. Type 301 full-hardened stainless steel has larger maximum tip deflections which would help to maximize the amount of flow through the reed valves. The slight advantage provided by the spring steel in terms of natural frequency is not enough to 
overcome the concern over its possible magnetic affects. The nonmagnetic type 301 full-hardened stainless steel is used which has a slightly lower natural frequency than carbon 1095 BT\&P spring steel but will allow more flow through the reed valve at maximum stress.

\subsubsection{Magnetic Circuit}

In an ideal magnetostrictive transducer, the magnetic induction through the rod would be perfectly uniform throughout its length. Considering the low relative permeability of Terfenol-D, approximately $\mu_{r}=5$, and the relative permeability of steel in the thousands, the path of the magnetic flux is a critical design feature of the Terfenol$\mathrm{D}$ transducer in order to magnetize the rod in the most efficient manner. The key pieces in the magnetic circuit consist of the Terfenol-D rod, stack Alnico V magnets and corresponding magnetic steel pieces (adjustor cap, Terfenol-D seat, piston, piston guide ring). It is noted that only the piston piece contacting the Terfenol-D is made out of magnetic steel.

The 4.5 in long stack of Alnico $\mathrm{V}$ magnets provides a biased magnetic field of approximately 130 Oe $(10.7 \mathrm{kA} / \mathrm{m})$ through the Terfenol-D, as illustrated in the quasistatic strain vs. magnetic field curve in Figure 4.8. The minor loop illustrates the maximum applied current (2.5 A peak to peak) that is in the linear operating range of the Terfenol-D transducer. If the current is to be increased, a DC current must be applied in order to increase the magnetic bias through the Terfenol-D to remain in the its linear region. The magnets are clamped between the piston guide ring and the adjustor cap, both of which are made out of 1020 steel. The seat and piston are made out of 1144 stress proof steel; they direct the magnetic flux path 


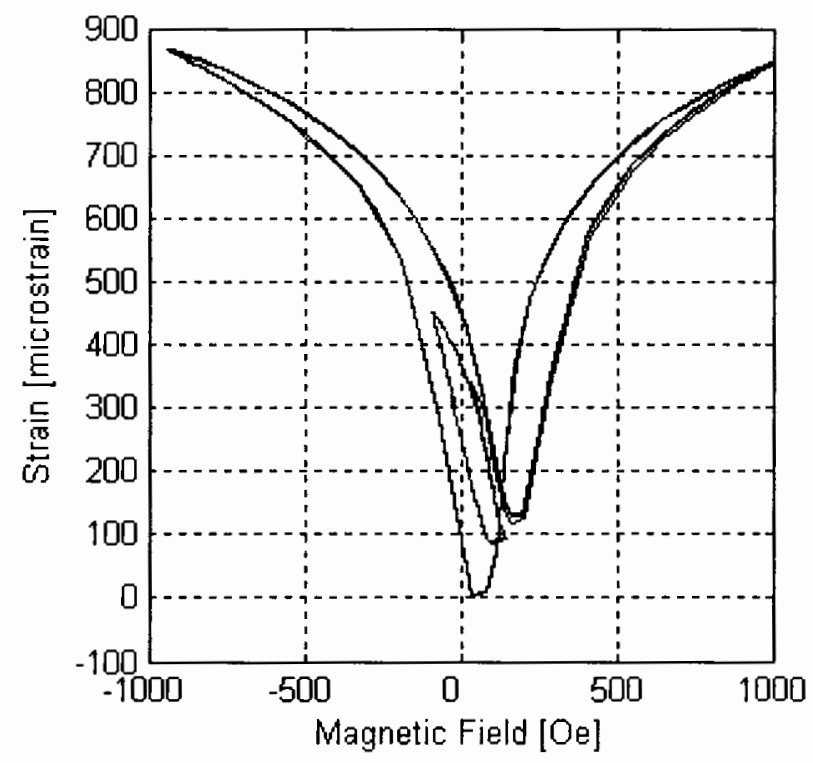

Figure 4.8: Strain vs. magnetic field (butterfly curve) for the Terfenol-D transducer illustrating the magnetic bias delivered by the Alnico $\mathrm{V}$ magnet stack.

through the Terfenol-D. It is noted that two minor air gaps are required to allow for sliding fits of the piston with respect to the piston guide ring and of the seat with respect to the adjustor cap.

The magnetic circuit is driven by a 860 turn coil made out of 20 AWG insulated copper wire. The coil has a field rating of $95 \mathrm{Oe} / \mathrm{A}$, a $\mathrm{DC}$ resistance of $1.9 \Omega$ and an inductance of $1.30 \mathrm{mH}$ with the Terfenol-D rod inside. The ID of the coil is slightly larger than that of the Terfenol-D rod to ensure that there is no contact while the length is slightly larger than that of the rod to ensure that the rod is not exposed to the drop off in magnetic field at the ends of the coil. The coil is wound with a variable-speed electric motor and held together with DURALCO 4525 epoxy resin and hardener. The epoxy also helps to conduct heat away from the Terfenol-D. 
In order to quantify the magnetic induction $B$ and the magnetic flux lines through the Terfenol-D rod, the magnetic circuit was analyzed in the magnetic finite element package Finite Element Method Magnetics (FEMM) Version 3.4. This FEA package utilizes a symmetrical solver that rotates a slice of the transducer about the axis of the transducer, so only a half of a cross-section of the transducer is drawn. The magnetic relative permeability for each material is input to the model while a magnetic moment on the Alnico stack provides the bias magnetic field. The software calculates the variation of the magnetic induction along the axis of the Terfenol-D rod due to the biased magnetic field induced by the Alnico stack, wee Figure 4.9. Since the Terfenol-D rod is relatively large, there is a dip in the magnetic induction in the middle portion of the rod. This behavior is unavoidable due to the high reluctance of the rod in the magnetic circuit. A plot of the magnetic flux lines and density (color) plot of the magnetic induction is given in Figure 4.10. It is observed that the magnetic circuit does not exactly direct all of the flux lines through the Terfenol-D rod; flux lines are escaping through the preload adjustor and the piston guide.

\subsubsection{Hydraulic Interface}

In interfacing the Terfenol-D transducer with the hydraulic oil, the most important design aspects deal with the type of fluid used and with proper hydraulic sealing. In choosing the type of hydraulic fluid, the bulk modulus is of great importance. The bulk modulus is defined by

$$
\beta=\frac{\Delta P}{\Delta V / V}
$$




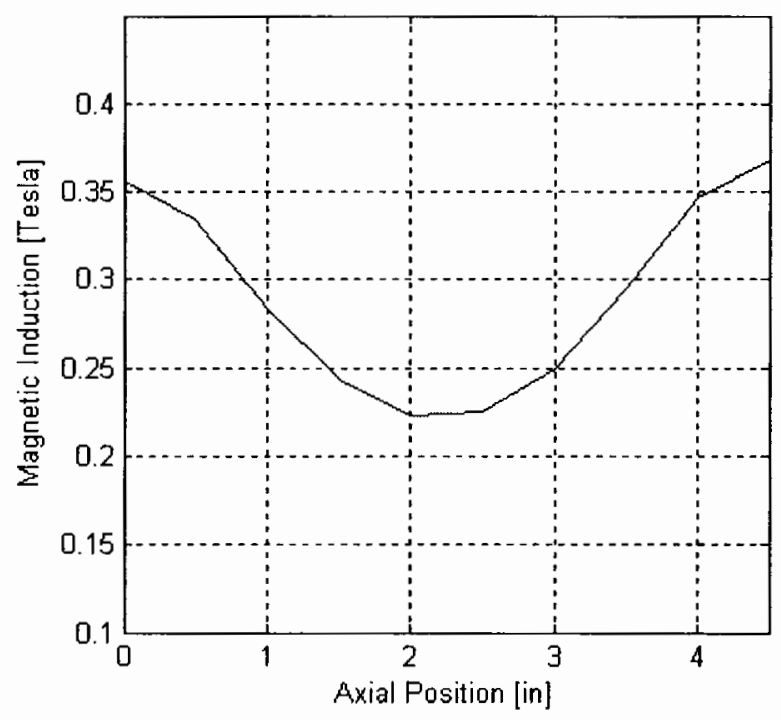

Figure 4.9: Magnetic induction along the length of the Terfenol-D rod, computed by FEMM for the magnetic circuit of the transducer.

where $V=A_{i} h$ is the volume of the chamber and $\Delta V=A_{i} \Delta h$ is the change in the chamber volume. The change in pressure inside the chamber $\Delta P$ is thus expressed as

$$
\Delta P=\frac{\Delta h}{h} \beta .
$$

A hydraulic fluid with a large bulk modulus is desirable because for the same change in chamber height $\Delta h$ due to magnetostriction of the Terfenol-D, a fluid with a larger bulk modulus will create a greater pressure differential.

Any leak introduces losses to the transducer and decreases the efficiency of the pump. The diaphragm and piston couple the Terfenol-D with the fluid. Wherever a metal to metal connection occurs in the pump head, aside from the reed valves, static O-rings are used to isolate the fluid in the pumping chamber. These static O-rings are not exposed inside the pumping chamber and thus add no compliance to the system. 


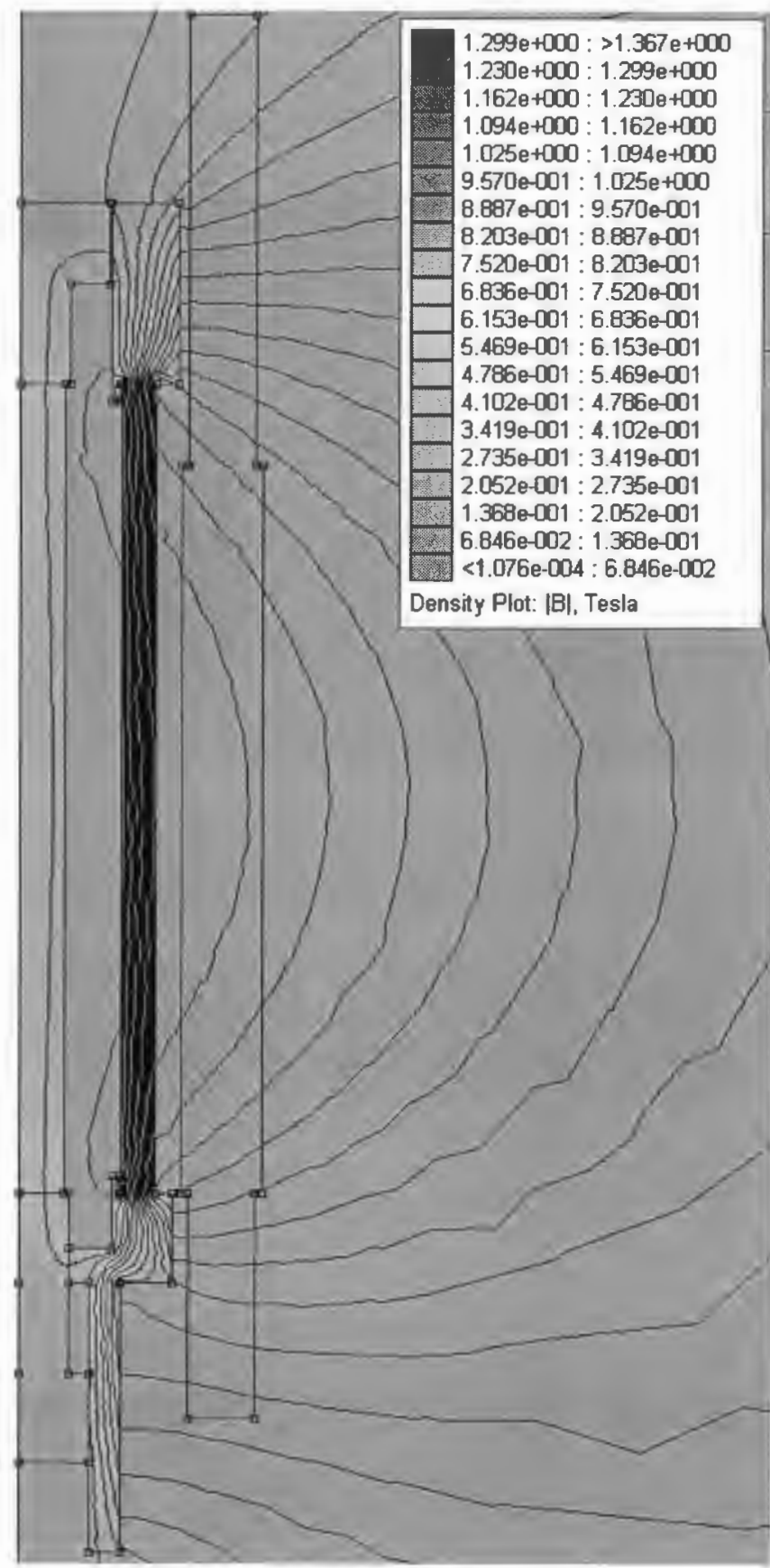

Figure 4.10: FEMM v 3.4 calculation of the magnetic flux lines and density of the magnetic induction in the magnetic circuit of the Terfenol-D pump. 
Standard O-ring face-seal (ORFS) hydraulic fittings are used for the IN/OUT ports on the pump head. ORFS hydraulic fittings are chosen over pipe thread fittings in order to decrease the size of the pump head. These fittings also allow for a uniform transition between the hydraulic steel lines connected to the actuator and the fluid paths inside the pump head. If pipe thread fittings had been used, a step in the flow path would be unavoidable and would introduce extra turbulence into the fluid network.

\subsection{Experimental Setup}

The harmonic and broadband testing performed on the transducer requires the same operating conditions in order to obtain meaningful data. As mentioned previously, any air trapped inside the transducer will greatly reduce the efficiency of the pump. The method for degassing the hydraulic oil is employed for all tests as detailed next. The preload on the Terfenol-D, bias pressure and temperature are all variables that must be held constant during testing; methods for obtaining these constants are described in the following sections.

\subsubsection{Removal of Entrained Air}

Inducing a vacuum on the hydraulic fluid removes any air bubbles and entrained gases in the fluid. A vacuum pump capable of pulling $29 \mathrm{mmHg}$ was purchased and is attached to top of the accumulator shown in Figure 4.11. Initially all manual valves are open. The pumping chamber is only exposed to the vacuum for a short period of time to remove the larger air bubbles and is then isolated from the vacuum by manual check valves 1 and 2 above the pump head. Some of the O-ring glands that seal the pumping chamber are designed for external pressure gradients. When the 


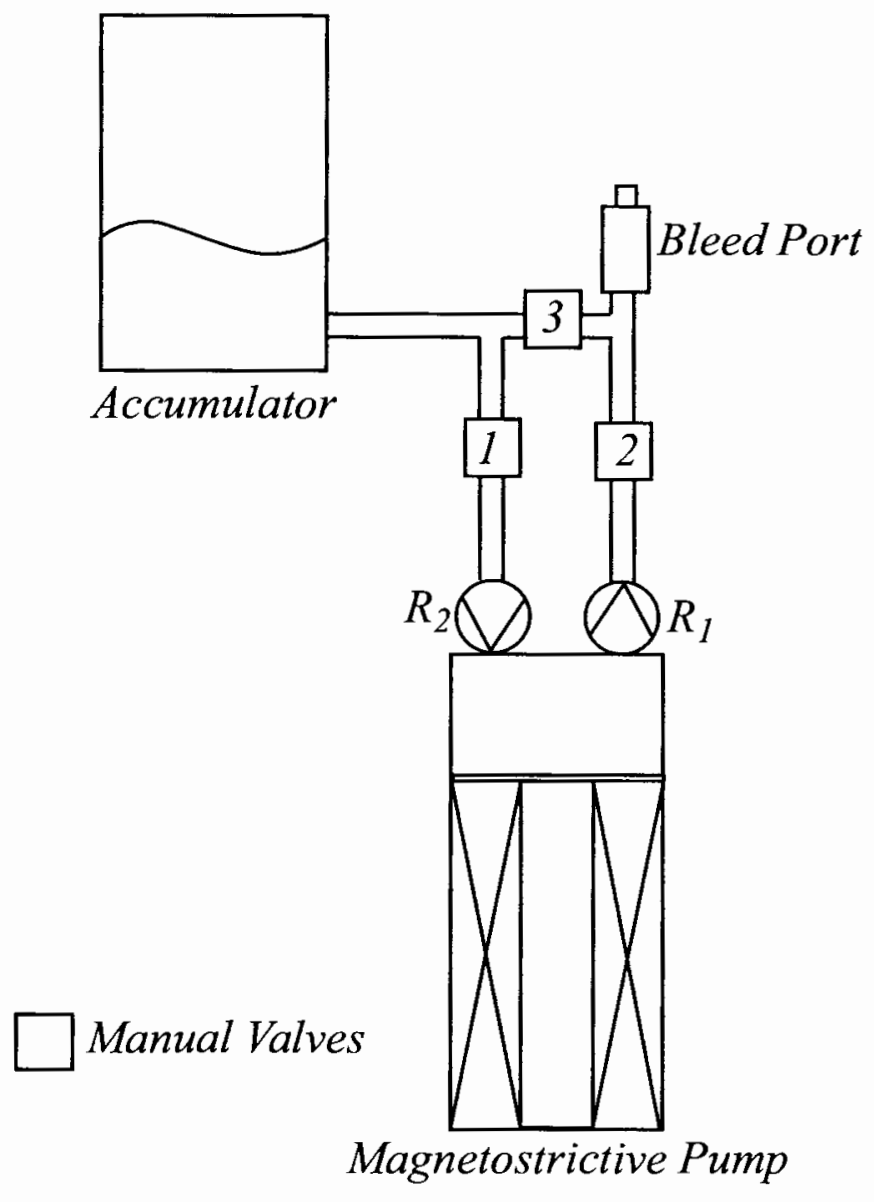

Figure 4.11: Schematic illustrating the setup for removal of air bubbles and entrained air.

pressure inside the chamber is less than atmospheric pressure, air will start to seep into the pumping chamber underneath the O-rings and the maximum vacuum that the pump is able to pull will not be reached. Manual valves 1 and 2 separate the pump head from the vacuum and the pumping chamber is flushed after the fluid is degassed. The pump is run for an hour or two to ensure that all of the air bubbles and entrained gases are removed. 
After the vacuum is removed, a nitrogen tank is connected to the accumulator, the bleed port is opened and manual valve 3 is closed. Rubber tubing is connected to the bleed port to direct the fluid to a container. Pressure is applied to the system which pushes the degassed fluid through the pumping chamber, pushing any remaining air bubbles out of the chamber. The fluid inside the chamber is replenished through this process. Air is visible at the bleed port as fluid is pushed through the system. Fresh fluid is continually pushed through the chamber until no more air is seen escaping from the system. This process of air removal is repeated as many times as necessary.

\subsubsection{Terfenol-D Preload}

Figure 4.12 illustrates the effect of increasing the amount of prestress on the butterfly curves (strain $\epsilon$ vs. magnetic field $H$ ) of Terfenol-D. In order to reduce the input power, a prestress of $1 \mathrm{ksi}$ is chosen for this transducer as it allows for maximum strain at the lowest field. This prestress corresponds to $190 \mathrm{lbs}$ of force for a diameter of 0.5 in. The $190 \mathrm{lbs}$ is applied by measuring the change in strain of the Terfenol-D rod. A strain gage is mounted to the rod and the required strain that yields a $190 \mathrm{lb}$ force is found by using Hooke's law,

$$
\begin{aligned}
F_{\text {pre }} & =k_{t} x_{0}=\frac{E_{y}^{H} A_{t}}{L_{t}} x_{0} \\
\epsilon_{0} & =\frac{x_{0}}{L_{t}}=\frac{F_{p r e}}{E_{y}^{H} A_{t}},
\end{aligned}
$$

where $F_{\text {pre }}$ is the preload, $x_{0}$ is the change in length under the preload and $\epsilon_{0}$ is the change in strain under the preload. The Etrema Products data sheet for Terfenol-D gives a range of $25-35 \mathrm{GPa}$ for the Young's modulus. The resulting range in strain is 197-275 $\mu \epsilon$ depending on the Young's modulus. 


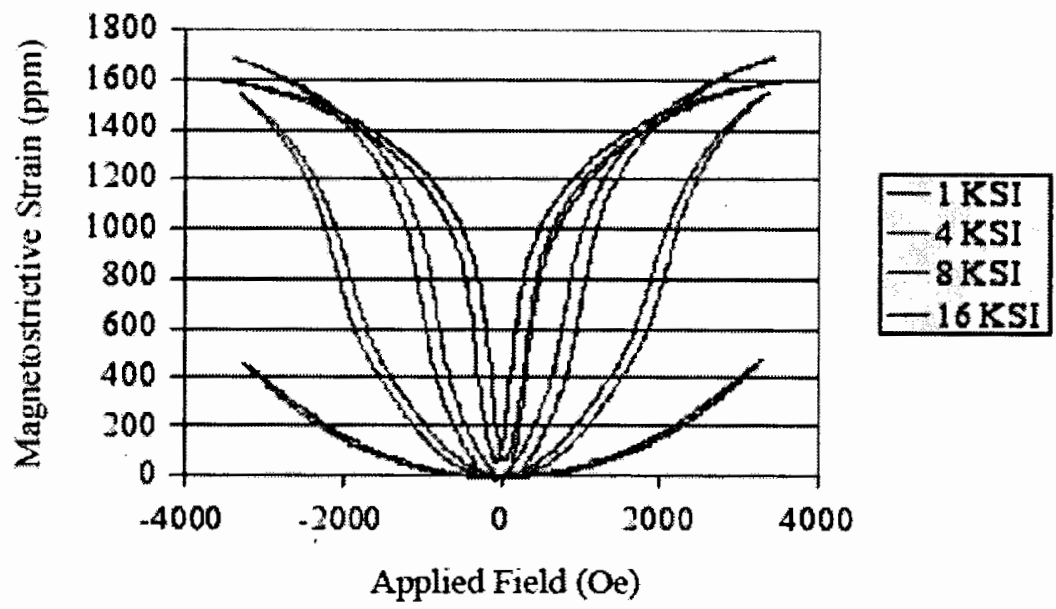

Figure 4.12: Characteristic strain versus magnetic field curves for various prestresses of Terfenol-D.

The preload is affected by both turning the preload bolt and the amount of bias pressure on the hydraulic oil. Initially the bolt compresses the rod just enough to ensure contact with the piston. The bias pressure is then added to the fluid and the bolt is adjusted accordingly to reach the required strain. During testing, the acceleration must be monitored to be sure that the driving conditions will not unload the Terfenol-D. If the acceleration is large enough, the piston will separate from the Terfenol-D and smack back down on the rod causing damage. The maximum acceleration allowed is calculated by

$$
a_{m a x}=\frac{F_{p r e}}{m_{e f f}}
$$

where $m_{\text {eff }}$ is the effective mass of the Terfenol-D rod, piston and diaphragm. The piston acceleration must be kept below the maximum acceleration allowable which is 750 g's. 


\begin{tabular}{|c|c|c|}
\hline $\begin{array}{c}\text { Bias Pressure } \\
{[\mathbf{p s i}]}\end{array}$ & $\begin{array}{c}\text { SS Pressure } \\
{[\mathbf{p s i}]}\end{array}$ & $\begin{array}{c}\text { Pressure Differential } \\
\text { [psi] }\end{array}$ \\
\hline \hline 100 & 895 & 795 \\
\hline 125 & 955 & 830 \\
\hline 150 & 1080 & 930 \\
\hline 200 & 1150 & 950 \\
\hline 250 & 1205 & 955 \\
\hline
\end{tabular}

Table 4.4: Effect of various bias pressures on pump performance for a $1 \mathrm{~Hz} / 20 \mathrm{~A}(0$ to peak) sinusoidal input.

\subsubsection{Bias Pressure}

The bias pressure on the hydraulic fluid has two purposes other than adding prestress to the rod: (i) aids in filling the pumping chamber by providing a constant source of pressure and (ii) prevents fluid cavitation. There exists an operating bias pressure that allows for optimal pump performance. A quasistatic signal $(1 \mathrm{~Hz} / 20 \mathrm{~A}$ peak to peak) is input to the transducer with the output port blocked. The steadystate pressure attained is recorded for each bias pressure and the results are given in Table 4.4. The pressure differential is defined as the difference between the maximum steady-state pressure and the bias pressure. It is noted that the nitrogen tank which applies the bias pressure is limited to 250 psi which correspondingly gives the maximum steady-state pressure differential. All subsequent testing will have an operating bias pressure of 250 psi even though a higher bias pressure may result in a larger pressure output. 


\begin{tabular}{|c||c|}
\hline Coefficient of Thermal Expansion & $12 \mathrm{ppm} /{ }^{\circ} \mathrm{C}$ \\
\hline Specific Heat & $0.35 \mathrm{~kJ} / \mathrm{kg}-\mathrm{K}$ \\
\hline Thermal Conductivity & $13.5 \mathrm{~W} / \mathrm{m}-\mathrm{K}$ \\
\hline
\end{tabular}

Table 4.5: Thermal coefficients of Etrema Terfenol-D.

\subsubsection{Temperature}

Since the material properties of Terfenol-D vary with temperature (see Table 4.5) the temperature inside the transducer must be kept at relatively the same temperature on order to obtain accurate measurements. Cooling of the transducer is essential. The spacing between test runs is determined by the time required to cool the transducer below a specific temperature. A thermocouple attached to the drive coil monitors the transducer temperature. The transducer is cooled below $80^{\circ} \mathrm{F}$ before each test to limit the thermal effects of the rod.

\subsection{Data Collection}

Experimental data of the transducer requires acquisition of the electrical, mechanical and fluid variables. Accurate measurements of transducer variables is a necessity in order to properly quantify the output of the system. A range of sensors is used to acquire the necessary data.

For proper application of the mechanical preload on the Terfenol-D rod, quantification of the system pressure and Terfenol-D strain is required. The system pressure is measured by Sensotec TJE 5000 psi pressure sensors located on either side of the pump head. These pressure sensors carry a sensitivity of $1 \mathrm{mV} / \mathrm{psi}$ and require a DC excitation voltage between 11-24 V. The bandwidth of the pressure sensors is 
$2 \mathrm{kHz}$. A Vishay Micro-Measurements precision strain gage (type EA-06-062AQ-350) is bonded to the Terfenol-D rod and aligned to measure the strain in the axial direction. The strain is output through a Vishay P-3500 Strain Indicator with a calibration factor of $0.157 \mathrm{mV} / \mu \epsilon$.

A UniMeasure LX-PA Series string potentiometer is attached to the hydraulic cylinder to measure displacement. The velocity is calculated by the derivative of this acquired data, from which the volume flow rate is calculated through multiplication of the velocity and cross-sectional area $A_{o}$ of the hydraulic cylinder. The string potentiometer has a maximum supply voltage $25 \mathrm{~V}$ (AC or DC) and a sensitivity of $48.548 \mathrm{mV} / \mathrm{V} / \mathrm{in}$, which for a supply voltage of $20 \mathrm{~V}$ is $971 \mathrm{mV} / \mathrm{in}$.

A PCB Piezotronics shear accelerometer (model number 353B15) is attached to the piston in order to capture the dynamic motion of the effective mass (piston, diaphragm, Terfenol-D) up to $10 \mathrm{kHz}$. The accelerometer signal $(10.99 \mathrm{mV} / \mathrm{g})$ is conditioned and read through the data acquisition system with a $4 \mathrm{~mA}$ constant current source. The input signal to the drive coil is amplified by an AE Techron LVC 5050 Linear Amplifier which is equipped with corresponding output voltage and current monitors. These monitors output voltage signals which are proportional to the magnitude of the actual signal. The voltage monitor has a sensitivity of $50 \mathrm{mV} / \mathrm{V}$ while the sensitivity of the current monitor is $167 \mathrm{mV} / \mathrm{A}$. The output voltage to current ratio is the measured electrical drive-point impedance $Z_{e e}$ which is the primary focus of the dynamic testing of the transducer.

The hardware used to acquire all experimental data is the Data Physics Quattro which works with the SignalCalc 240 Dynamic Signal Analyzer software. The Quattro is capable of acquiring up to four input channels that accept up to $+/-10 \mathrm{~V}$. The 
output channel connected to the amplifier can output any desired signal up to $+/-$ $5 \mathrm{~V}$ and is sent to the amplifier. The software can perform frequency response function analysis of the input signals for the desired combinations.

\subsection{Test Parameters}

Harmonic and broadband testing of the Terfenol-D transducer is performed in order to quantify its performance characteristics. Testing is used to determine the maximum attainable pressure and maximum no-load actuator velocity and to characterize the electrical drive-point impedance. The following discussion outlines the experimental setup designed to obtain the desired variables.

Dynamic testing of the Terfenol-D transducer is performed in order to quantify the electromechanical coupling of the transducer by obtaining $Z_{e e}$. Swept sine tests are run from $10 \mathrm{~Hz}-20 \mathrm{kHz}$ with a constant input voltage. The magnitude of the voltage is small enough so that the transducer is always operating under proper magnetic bias. The theoretical maximum attainable pressure of the Terfenol-D transducer occurs under blocked output conditions [4]. Tests are run under blocked output operating conditions for both with and without the output reed installed. The output manual valve is closed while the input manual valve is open to ensure a constant pressure source for the pumping chamber. Tests with the output reed removed allow for quantification of the pressure response of the transducer by allowing the pressure to return to the bias pressure. Testing with the reed valve installed is also conducted with the output manual valve closed. Frequency response functions of transducer voltage to current $\left(Z_{e e}\right)$ and piston acceleration per current are obtained along with the magnitude response for each input variable. 
Harmonic pressure testing is performed under blocked output conditions. The recorded variables are the blocked pressure near the output reed valve, piston acceleration, and input current and voltage. The time is also recorded for all harmonic time domain testing by the SignalCalc software. Two separate tests are performed. First, in order to quantify the frequency limitation of the reed valves, harmonic testing with 4 A peak to peak input is performed at various frequencies in an effort to trace the dynamic response of the pressure found through broadband testing. Secondly, to quantify the maximum pressure at a larger drive current, a $20 \mathrm{~A}$ peak to peak input waveform is held constant for each test. After each run, the transducer is cooled, the output manual valve is opened and then closed to purge the chamber pressure to the system bias pressure. It is noted that the 0.015 in reed valves experienced difficulty in sealing, thus for all subsequent testing, the 0.015 in reed valves are not discussed.

Harmonic actuation testing of the EHA is performed for no applied load on the actuator. The string potentiometer measures the displacement of the hydraulic cylinder and the no-load velocity is determined by analyzing data after each test. Again current is held constant but is a waveform of 10 A peak to peak. The no-load condition of the actuator permits larger amplitudes of the magnetostrictive vibrations which results in much larger accelerations. In order to assure that the acceleration does not unload the Terfenol-D rod, the input current is decreased which lowers the magnitude of the Terfenol-D vibrations and thus the acceleration. The input current, output pressure, piston acceleration and actuator displacement are the measured variables for each test. The results from the no-load testing give insight into the limitation of the complete EHA. 


\section{CHAPTER 5}

\section{RESULTS AND DISCUSSION}

Experiments are conducted on the Terfenol-D transducer in order to establish the performance parameters of the pump, identify material properties of the transducer and validate the model simulations. Dynamic testing of the transducer quantifies the electrical drive-point impedance and system resonance and, with implementation of the dynamic system model, also provides insight into specific transducer characteristics. Harmonic tests in the time domain identify the maximum pressure differential and the frequency limitations of the reed valves and complete EHA.

\subsection{Dynamic Testing and Model Simulation}

In order to determine the dynamic characteristics of the Terfenol-D transducer, stepped-sine testing is performed for frequencies up to $20 \mathrm{kHz}$. In a stepped-sine test, the analyzer directs an internal signal generator to create a steadysine wave at one frequency. The system response is measured just at that frequency. The data point is stored, the signal generator is adjusted to a new frequency, and after ap pause while the system transient response settles out, the measurement is repeated for the new point. Frequency response functions are built up from hundreds of individual measurements, each at a single point. A number of bands may be used in order to fine 


\begin{tabular}{|c||c|}
\hline Sensor & $\begin{array}{c}\text { Bandwidth } \\
{[\mathrm{kHz}]}\end{array}$ \\
\hline \hline Pressure Sensor & 2 \\
\hline Strain Indicator & 4 \\
\hline Accelerometer & 10 \\
\hline Voltage Monitor & 20 \\
\hline Current Monitor & 20 \\
\hline
\end{tabular}

Table 5.1: Bandwidths of the various sensors used in testing.

tune the response around a certain frequency. Although the transducer is being tested up to $20 \mathrm{kHz}$, some of the sensors have bandwidth limitations far below this frequency. Table 5.1 lists the maximum operating frequency for obtaining accurate data for the various sensors used during these tests. Two sets of tests are performed, both with and without the output reed valve installed. As mentioned in Chapter 4 the dynamic testing without the output reed valve allows for a measure of the pressure response of the transducer. Both sets of tests are completed with the output manual valve closed. Input current, input voltage and piston acceleration are recorded for both tests while output pressure is recorded for the test without the output reed valve. Output pressure is not recorded for the test with the output reed valve installed because this would be a measure of the pressure oscillations outside of the reed valve, which are minimal. Figures 5.1-5.2 show the results for both tests. The dynamic data without the output reed valve installed illustrates resonant activity at approximately $1600 \mathrm{~Hz}$ and $5060 \mathrm{~Hz}$ in the magnitude and phase of the electrical impedance and also in the magnitude of the piston acceleration per input current transfer function. These motional affects correspond to peaks of $61.8 \mathrm{~g} / \mathrm{A}$ and $182.4 \mathrm{~g} / \mathrm{A}$ respectively. The pressure response shows an initial peak at $10 \mathrm{~Hz}$ followed by a decay in the pressure 
response and then a larger peak at $1600 \mathrm{~Hz}$. The tests with the output reed valve installed show only resonant motion at $5140 \mathrm{~Hz}$; the peak at $1600 \mathrm{~Hz}$ is removed when the output reed is installed. This loss of transduction illustrates the change in loading between tests and indicates an increase in the loading on the Terfenol-D rod when the output reed valve is installed. When the reed is removed, the fluid dynamics of the pump head changes and creates an unobstructed output cavity that allows pressure waves to reflect off of each other and amplify the pressure response at $1600 \mathrm{~Hz}$. When the output reed is installed, it prevents the fluid from acting in this manner. In both cases, the resonant motion near $5100 \mathrm{~Hz}$ is due to the mechanical resonance of the Terfenol-D transducer.

A modification is made to the dynamic system model to simulate the blocked output conditions of these tests with the output reed valve installed. The hydraulic cylinder is assumed to be attached to an infinite mass which would result in no actuator motion. A stepped sine simulation is performed on the dynamic system model using this modification. Frequencies from $10 \mathrm{~Hz}-20 \mathrm{kHz}$ are investigated with a frequency step of $50 \mathrm{~Hz}$. The step is refined to $10 \mathrm{~Hz}$ near the location of the transducer resonance. The location of the resonance in the model is dependent on the values of two transducer properties, the Young's modulus $E_{y}^{H}$ of Terfenol-D and the bulk modulus $\beta$ of the hydraulic fluid, which are respectively proportional to the Terfenol-D stiffness $k_{t}$ and fluid stiffness $k_{f}$. Initial model simulations assumed maximum values of $E_{y}^{H}=35 \mathrm{GPa}$ and $\beta=1.64 \mathrm{GPa}$. Under these assumptions, the model predicts a transducer resonance of $6250 \mathrm{~Hz}$ as illustrated in Figure 5.4. Also the peak to peak amplitude of the motional component of the model is much larger than in the experimental data. The initial assumptions simulate a system that is too 


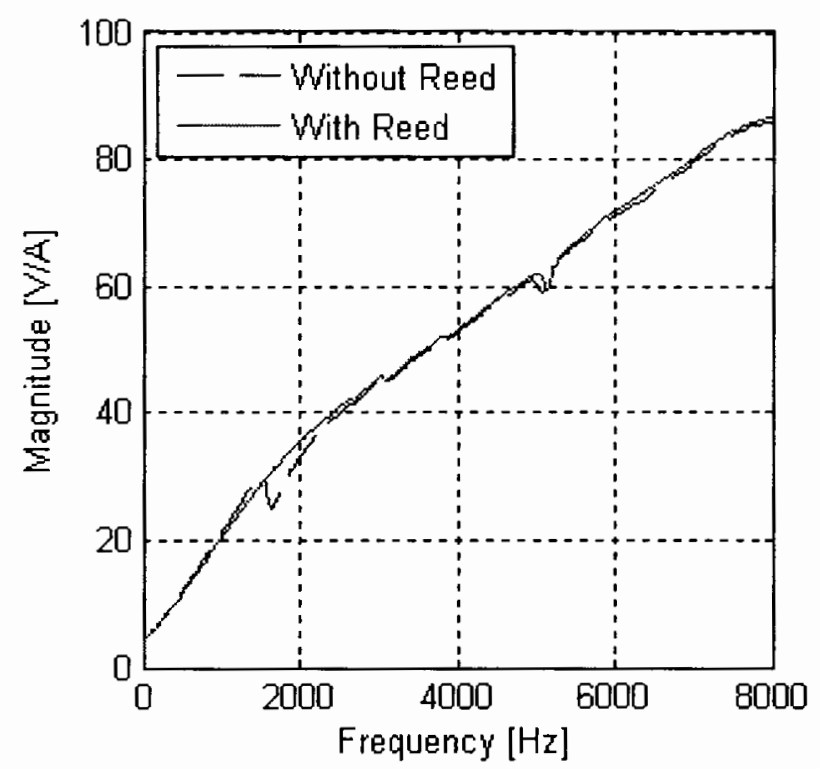

(a)

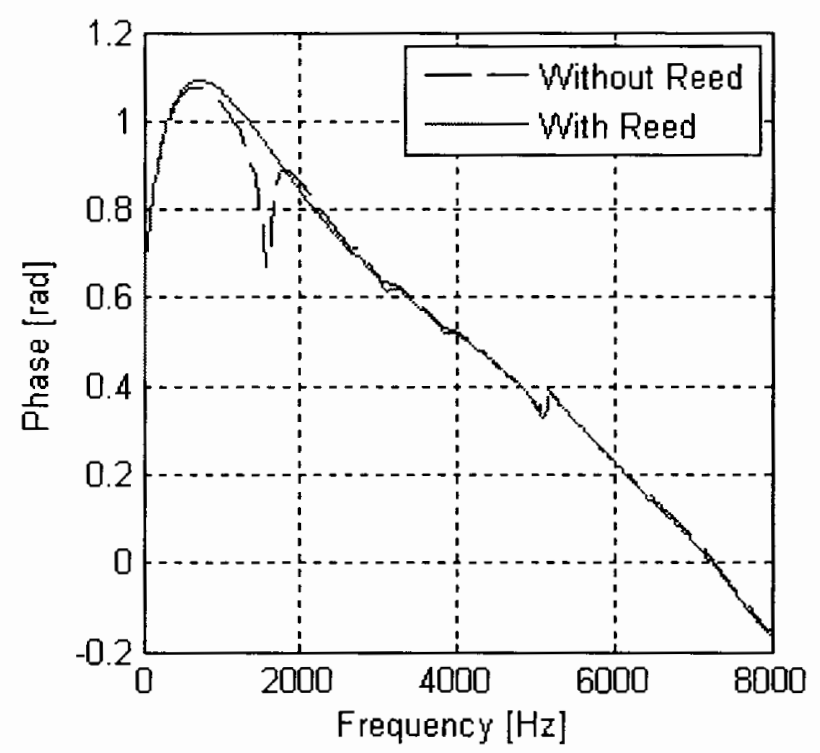

(b)

Figure 5.1: Results of stepped-sine testing of the Terfenol-D transducer: (a) Magnitude and (b) phase response of the electrical impedance frequency response function. 


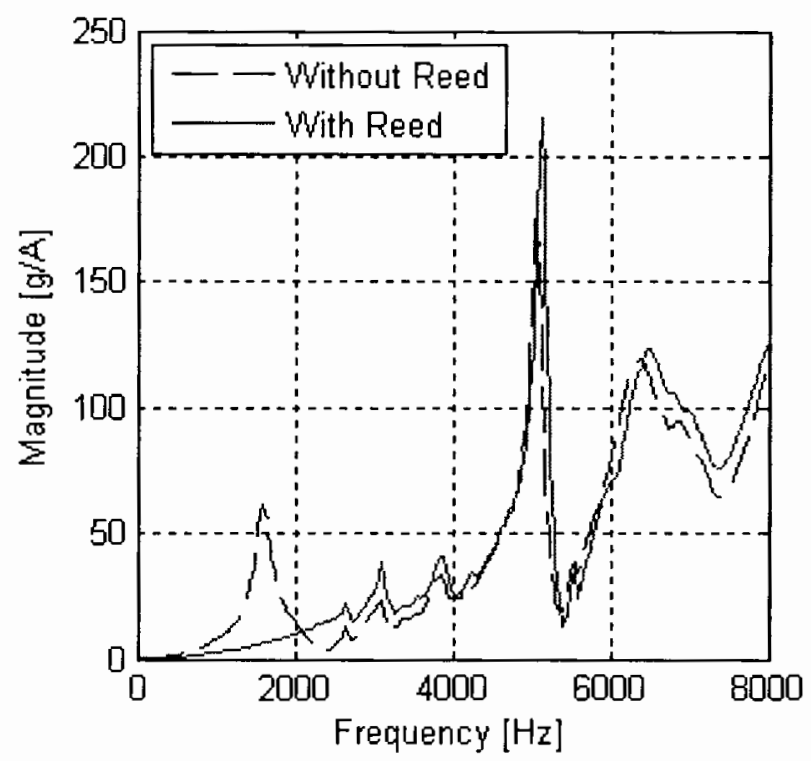

(a)

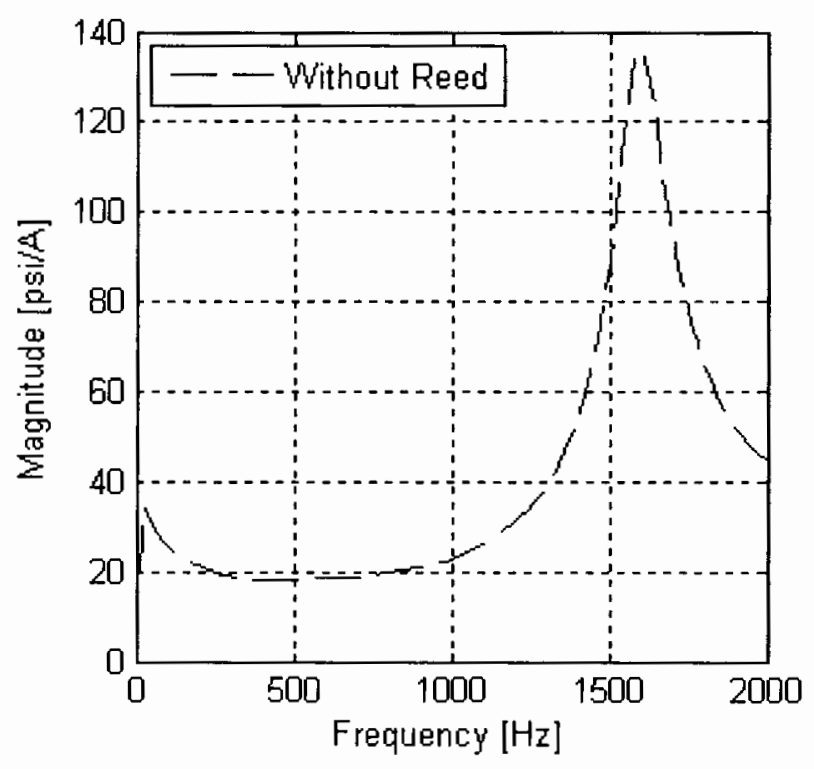

(b)

Figure 5.2: Results of dynamic testing of the Terfenol-D transducer: (a) Magnitude response of the acceleration/input current frequency response function and (b) magnitude response of the pressure/input current frequency response function. 


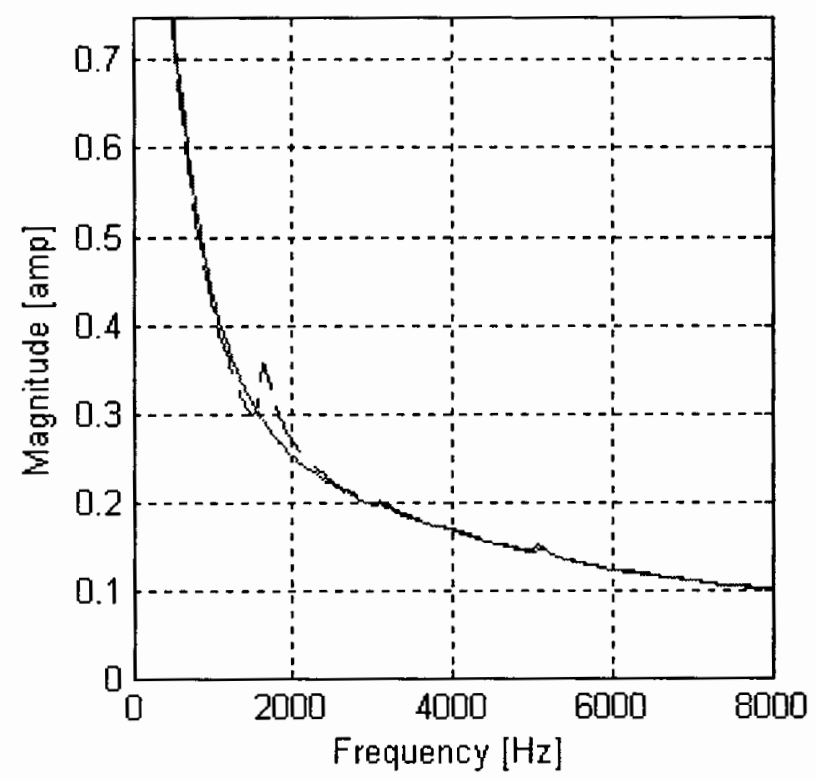

Figure 5.3: Magnitude response of the input current frequency response function.

stiff. The Young's modulus of Terfenol-D and the bulk modulus of the hydraulic fluid determine the Terfenol-D and fluid stiffness coefficients and these stiffnesses control the location of the motional component of the model since the effective mass is fixed. In order to obtain resonance near $5100 \mathrm{~Hz}$, the values of the material properties $E_{y}^{H}$ and $\beta$ need to be lowered in order to match the resonances. Lowering the bulk modulus becomes a measure of the effective bulk modulus $\beta_{\text {eff }}$ of the chamber which accounts for any compliance that the model overlooks, such as any air left in the chamber or structural compliances. Another source of compliance could be the small section of the diaphragm that is exposed between the piston and chamber ring. It is noted that the repeatability of the dynamic measurements is very high suggesting that the method of air removal is quite effective or that the slightest bit of air might be getting stuck 


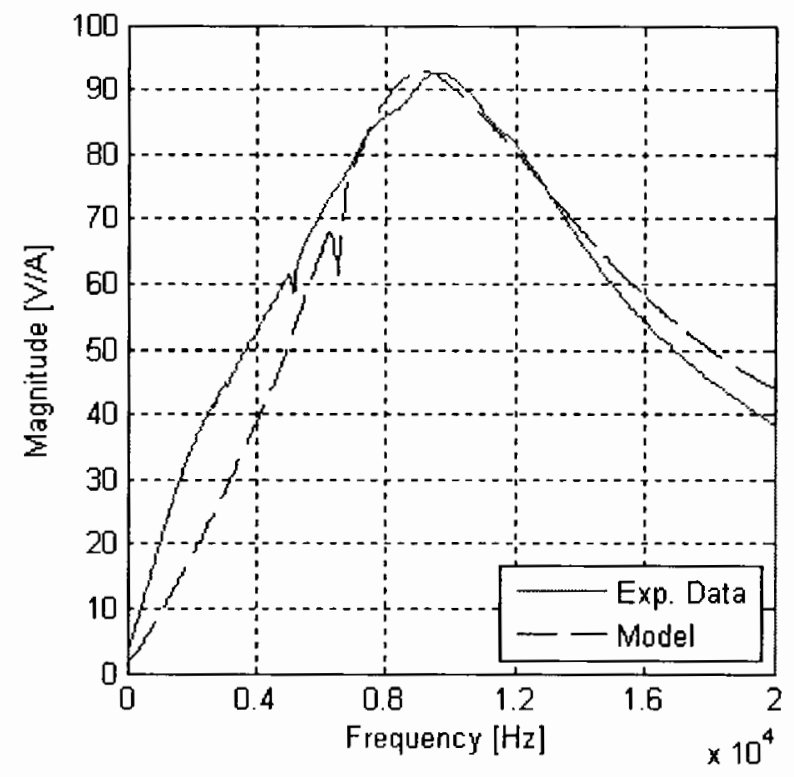

Figure 5.4: Magnitude of the electrical drive-point impedance for experimental data and the model. The model predicts a resonance of $6250 \mathrm{~Hz}$ while resoance of the Terfenol-D transducer is at $5100 \mathrm{~Hz}$.

somewhere inside the chamber every time. Changing the bulk modulus has a greater affect on the location of the transducer resonance and is lowered as needed while the Young's modulus of Terfenol-D typically is in the range of 25-35 GPa. The resulting combination of $E_{y}^{H}=30 \mathrm{GPa}$ and $\beta=0.93 \mathrm{GPa}$ produces the responses illustrated in Figures 5.5-5.6 which are plot against experimental data for comparison. The magnitude of the motional component is determined by the measure of the damping in the two degree of freedom model of the pump mechanical subsystem. The damping factor $\zeta_{t}$ has a more critical effect on the magnitude in that it must be held at a value lower than 0.13 . For $\zeta_{t}>0.13$, the ' $\mathrm{Z}$ ' shape due to the motional component in the model is completely flattened out. The damping factor $\zeta_{f}$ has the inverse effect on the motional component than $\zeta_{t}$ does. Increasing $\zeta_{f}$ increases the magnitude of the 
motional component. The corresponding values for $\zeta_{t}$ and $\zeta_{f}$ that give the results in Figures 5.5-5.6 are 0.09 and 0.55. A complete list of the electrical, mechanical and fluid parameters used in the model is given in Appendix A

The large peak at $9500 \mathrm{~Hz}$ is due to the capacitive nature of the blocked electrical impedance. The model calculates the inductance $L_{b l o c k}$ through the mechanical and physical parameters of the Terfenol-D rod and the capacitance is chosen so that the peak in the electrical drive-point impedance in the model is located at $9500 \mathrm{~Hz}$. The corresponding inductance $L_{b l o c k}$ and capacitance $C$ that give the location of this peak are $1.30 \mathrm{mH}$ and $209 \mathrm{nF}$. The experimental data deviates from the model from $10 \mathrm{~Hz}$ to approximately $7000 \mathrm{~Hz}$. This deviation is evident in the phase response as the phase in the experimental data never reaches the maximum phase of the model. This deviation is also illustrated in the Nyquist plot of the total electrical impedance in Figure 5.7. The deviation is expected due to the numerous assumptions made during derivation of the linear model. Terfenol-D is a nonlinear material and is being modeled linearly under the assumption that it is in its linear operating range. The inductance and capacitance of the fluid lines are ignored. Also, the model does not account for any turbulent or recirculation of the flow dynamics through the pump head.

In order to quantify the magnetomechanical coupling of the Terfenol-D transducer and model, the dynamic method for calculating the conversion between magnetic and elastic energies is used by interpreting the locations of the resonant $f_{r}$ and antiresonant $f_{a r}$ frequencies as in equation (3.27),

$$
k_{e f f}^{2}=1-\left(\frac{f_{r}}{f_{a r}}\right)^{2} .
$$




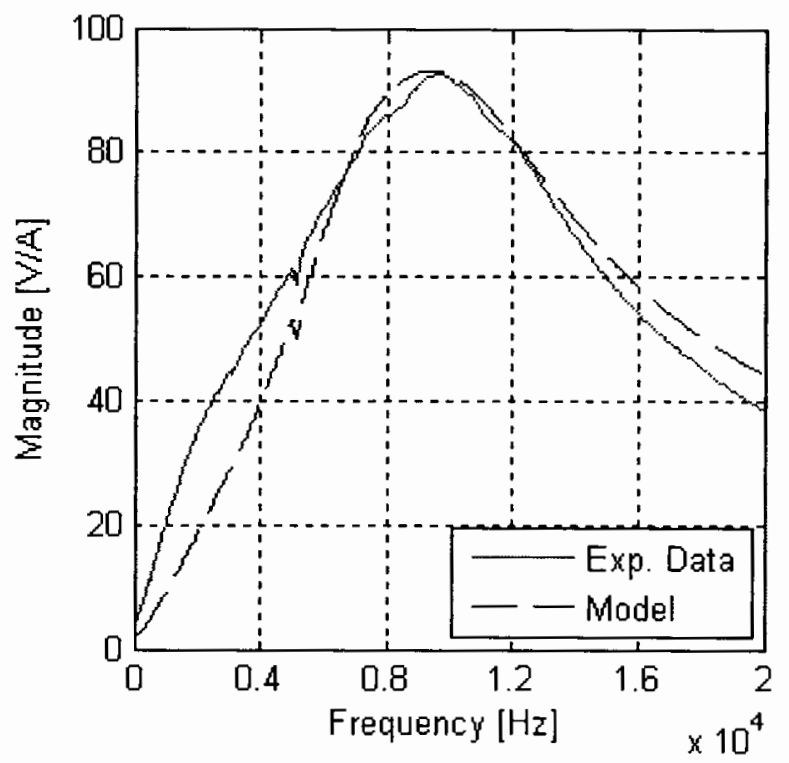

(a)

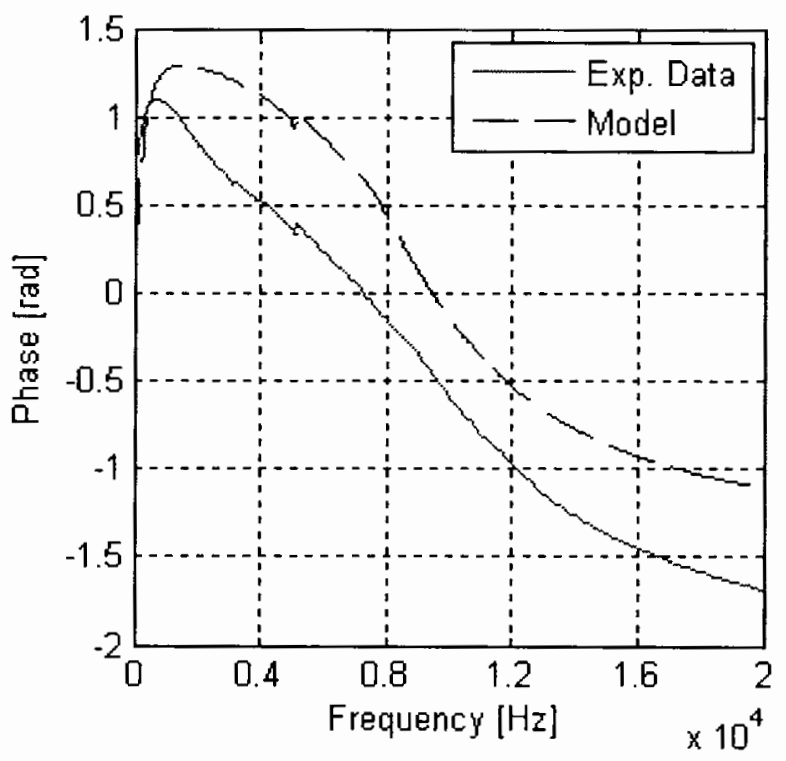

(b)

Figure 5.5: Comparison of model simulation and dynamic testing of the Terfenol-D transducer: (a) Magnitude and (b) phase response of the electrical impedance $Z_{e e}$. 


\begin{tabular}{|c||c|c|c|}
\hline & $f_{r}[\mathbf{H z}]$ & $f_{a r}[\mathbf{H z}]$ & $k_{\text {eff }}$ \\
\hline \hline Experimental Data & 5020 & 5140 & 0.215 \\
\hline System Model & 5000 & 5130 & 0.224 \\
\hline
\end{tabular}

Table 5.2: Location of resonance, antiresonance and the respective magnetomechanical coupling coefficients for the experimental data and the dynamic system model calculated from equation (3.27).

The effective magnetomechanical coupling coefficient for the experimental data and the dynamic system model are 0.215 and 0.224 , respectively, as illustrated in Table 5.2.

\subsection{Harmonic Testing and Model Simulation}

\subsubsection{Blocked Output Testing}

Harmonic time domain testing is performed on the Terfenol-D transducer with the output manual valve closed, simulating the blocked output condition which gives the theoretical maximum pressure. The first of two sets of blocked harmonic tests is intended to trace the pressure response given in Figure 5.3(b) for a constant current of $4 \mathrm{~A}$ peak to peak in order to find the frequency limitation of the reed valves. This input current is chosen to allow for maximum pressure response but not produce accelerations on the piston that would unload the Terfenol-D rod. The input voltage, input current and blocked pressure are measured for each frequency. Figure 5.8 shows the results from this harmonic testing alongside the stepped-sine pressure response. The blocked harmonic tests follow the trend of the dynamic pressure trace up until $1000 \mathrm{~Hz}$ where a drop in frequency occurs. The 0.005 in reed valves were designed in air to have a natural frequency of approximately $3000 \mathrm{~Hz}$. The drop in the range 
of usable frequencies for the reed valves is due to the shear loading effects of the hydraulic fluid.

The second set of blocked harmonic tests is to quantify the maximum blocked output pressure for a drive current of $10 \mathrm{~A}$ amplitude. Frequencies between 1-100 $\mathrm{Hz}$ are tested for the Terfenol-D transducer under blocked output conditions. As before the input voltage, input current and blocked pressure are measured for each frequency. The results are shown in Table 5.3 comparing maximum transducer pressure, leakage pressure and power consumption characteristic of the 0.005 in and the 0.010 in reed valves. The leakage pressure is defined as the difference in the absolute peak pressure and steady state pressure as in the output pressure time trace of the transducer in Figure 5.9. The pressures obtained with the 0.005 in reed valves are approximately twice the pressures obtained with the 0.010 in reed valves. The maximum pressure of 1100 psi above the bias pressure occurs at $10 \mathrm{~Hz}$ with a power consumption of $83.8 \mathrm{~W}$ for the 0.005 in reed valves. The pressure differential for the 0.005 in reed valves peaks at $10 \mathrm{~Hz}$ then decays as the frequency is increased while the maximum pressure for the 0.010 in reed valves is at the quasistatic frequency of $1 \mathrm{~Hz}$ and is followed by a decay in pressure with increasing frequency. The input power is calculated by

$$
P_{i n}=V_{R M S} I_{R M S} \cos \theta
$$

where the phase $\theta$ between the RMS voltage and current is obtained for each frequency by the phase response of $Z_{e e}$ provided in Figure 5.5(b). The input power to the transducer experiences a minimum at $25 \mathrm{~Hz}$ and then increases with increasing frequency.

A time trace at $10 \mathrm{~Hz}$ for the dynamic system model is performed using the system variables identified during the dynamic testing of the transducer and model. 
Figure 5.9 illustrates the time traces of the output pressure $P_{3}$ of the model and the blocked pressure of the Terfenol-D transducer for a $10 \mathrm{~Hz} / 10 \mathrm{~A}$ sinusoidal input. The experimental data shows small spikes in the pressure trace, which occur at $10 \mathrm{~Hz}$. These spikes in the data are due to pressure leaking back into the chamber before the reed valve is fully closed. The magnitude of the leakage in the steady state region of the pressure trace is $40 \mathrm{psi}$. As shown in Table 5.3, the magnitude of leakage pressure decreases as the frequency is increased. The model captures the trend of the harmonic data but lacks the pressure 'recoil' that is present in experimental data. The algorithm describing the fluid diode behavior in the model does not account for the pressure leaking beneath the reed as it is closing. An algorithm is derived based on the motion of the reed itself in an attempt to capture the pressure leakage. In theory this would account for pressure leakage but, in practice, this algorithm introduces discontinuities in the reed motion when introducing contact with the reed seat. These discontinuities make the model unstable. The model, as is, predicts the maximum attainable pressure assuming ideal fluid diode behavior of the reed valve.

\subsubsection{No-Load Testing}

The main focus of the harmonic no-load actuation tests is to locate the frequency limitation of the EHA and also quantify the maximum no-load velocity. An input current of $5 \mathrm{~A}$ amplitude is held constant for each test. A smaller current is used in the no-load testing because the change in transducer loading will allow for larger Terfenol-D deflections and thus larger accelerations. The current is decreased for precautionary measures. Table 5.4 gives the no-load velocities for frequencies tested up to $200 \mathrm{~Hz}$. There is a steep drop off in actuator velocity after $165 \mathrm{~Hz}$ with a peak 


\begin{tabular}{|c|c|c|c|c|}
\hline $\begin{array}{l}\text { Reed } \\
\text { [in] }\end{array}$ & $\begin{array}{c}\text { Frequency } \\
{[\mathrm{Hz}]}\end{array}$ & $\begin{array}{l}\text { SS Pressure Diff. } \\
{[\mathrm{psi}]}\end{array}$ & $\begin{array}{c}\text { Leakage Pressure } \\
{[\mathrm{psi}]}\end{array}$ & $\begin{array}{c}\text { Input Power } \\
{[\mathrm{W}]}\end{array}$ \\
\hline \multirow{6}{*}{0.005 in } & $\overline{1}$ & 965 & 113 & $\overline{88.1}$ \\
\hline & 5 & 1085 & 42 & 84.1 \\
\hline & 10 & 1100 & 40 & 83.8 \\
\hline & 25 & 1025 & 25 & 76.0 \\
\hline & 50 & 900 & 24 & 80.6 \\
\hline & 100 & 735 & 24 & 93.3 \\
\hline \multirow{6}{*}{$0.010 \mathrm{in}$} & $\bar{~} 1$ & $\overline{\overline{633}}$ & $\overline{84}$ & $\overline{99.5}$ \\
\hline & 5 & 581 & 64 & 92.9 \\
\hline & 10 & 534 & 57 & 86.7 \\
\hline & 25 & 495 & 31 & 82.0 \\
\hline & 50 & 483 & 25 & $87 . \overline{9}$ \\
\hline & 100 & 372 & 22 & 100.3 \\
\hline
\end{tabular}

Table 5.3: Steady state pressure differential, leakage pressure during reed closure and power consumption resulting from harmonic testing of the EHA at a bias pressure of $250 \mathrm{psi}$ and for sinusoidal input current with an amplitude of $10 \mathrm{~A}$.

at $160 \mathrm{~Hz}$. It is noted that the tests at or below $165 \mathrm{~Hz}$ have repeatable results but the same cannot be said for frequencies above $165 \mathrm{~Hz}$ where the discrepancy between tests is quite significant. Testing with 0.010 in reed valves resulted in no significant actuator motion. The stiffness of the 0.010 in reed valves limit the deflection of the reed and thus the volume flow and pressure output from the pump head. The hydraulic cylinder used in these tests has a maximum operating pressure of $2320 \mathrm{psi}$ with a $25 \mathrm{~mm}$ bore and $18 \mathrm{~mm}$ rod. The high pressure sealing of the piston contributes a large amount of friction to the actuator and increased difficulty in manually moving the piston is experienced when a bias pressure is applied to the hydraulic fluid. As illustrated in Figure 5.8 in the harmonic pressure response, a peak in the pressure response occurs at $10 \mathrm{~Hz}$ and the pressure produced between $100-200 \mathrm{~Hz}$ is not enough to overcome the force of friction in the hydraulic cylinder. The nonuniform forces of 


\begin{tabular}{|c||c|}
\hline $\begin{array}{c}\text { Frequency } \\
\text { [Hz] }\end{array}$ & $\begin{array}{c}\text { No-Load Velocity } \\
\text { [in/s] }\end{array}$ \\
\hline \hline 10 & 0.004 \\
\hline 25 & 0.078 \\
\hline 50 & 0.112 \\
\hline 75 & 0.185 \\
\hline 100 & 0.272 \\
\hline 150 & 0.316 \\
\hline 160 & 0.365 \\
\hline 165 & 0.331 \\
\hline 170 & 0.003 \\
\hline 175 & 0.055 \\
\hline 200 & 0.002 \\
\hline
\end{tabular}

Table 5.4: No-load velocities resulting from harmonic testing of the EHA at a bias pressure of $250 \mathrm{psi}$ and input current with an amplitude of $5 \mathrm{~A}$. Reeds used are 0.005 in thick.

friction accounts for the erratic behavior above $165 \mathrm{~Hz}$. This also accounts for the lack of actuator motion for tests with the 0.010 in reed valves installed. $165 \mathrm{~Hz}$ is the frequency limitation of the EHA but is not the limitation of the Terfenol-D transducer.

Research by a previous student in the Smart Materials and Structures Laboratory focused on fluid rectification with a magnetorheological (MR) valve network [27]. A no-load velocity of $0.325 \mathrm{in} / \mathrm{s}$ of a sinusoidal input produced by a hydraulic cylinder attached to a MTS load frame is obtained. The EHA in this research produces a no-load velocity of $0.365 \mathrm{in} / \mathrm{s}$ which is a slight improvement in performance but a major improvement in how it was obtained. This EHA is a self-contained system free from any external inputs, aside from electrical connections. 


\subsection{Results Summary}

The dynamic system model accurately predicts the maximum pressure of the Terfenol-D transducer, location of the transducer resonance and also the dynamic behavior up to $20 \mathrm{kHz}$. The model identifies important characteristics of the pump that could not be known otherwise, such as the effective bulk modulus, Young's modulus, damping ratios and drive coil capacitance. The dyanmic system model is a linear model of a nonlinear material. Terfenol-D is nonlinear in nature and assumptions are made to utilize these linear modeling techniques. The model also does not account for any turbulent effects or recirculation of the flow dynamics through the pump head and also ignores the inertia and capacitance of the hydraulic fluid in the fluid lines. Although the deviations from the linear dynamic system model and experimental data seem significant, the model accurately describes the locations and magnitudes of the dynamic activity of the Terfenol-D pump after taking into consideration all the assumptions that allowed linear modeling of the highly nonlinear magnetostrictive material Terfenol-D.

The thinnest reed valve allows for the maximum pump performance. The TerfenolD pump produces a maximum steady-state pressure differential of $1100 \mathrm{psi}$ at $10 \mathrm{~Hz}$ and $83.8 \mathrm{~W}$. The model captures the trend of the maximum pressure time trace but does not include the pressure 'recoil' due to the reed valve closing. The shear loading effects of the hydraulic oil slow the response of the reed valve creating this leak in the pressure trace due to fluid escaping beneath the reed as it closes. A lighter hydraulic fluid will decrease the effects of the shear loading due to the fluid and increase the reed valve response and at the same time reduce the amount of pressure leakage as the reed valve is closing. The reed valves have a maximum operational frequency of 
$1000 \mathrm{~Hz}$. The EHA provides a maximim no-load velocity of $0.365 \mathrm{in} / \mathrm{s}$ at $160 \mathrm{~Hz}$ and has a frequency limitation at $165 \mathrm{~Hz}$. The frequency limitation of the EHA is due to the high frictional forces of the hydraulic cylinder. 


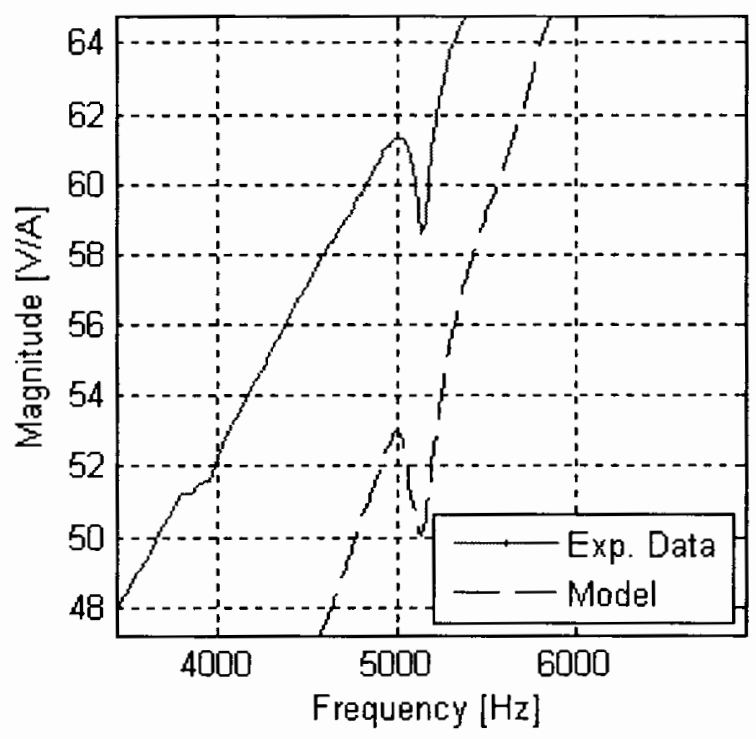

(a)

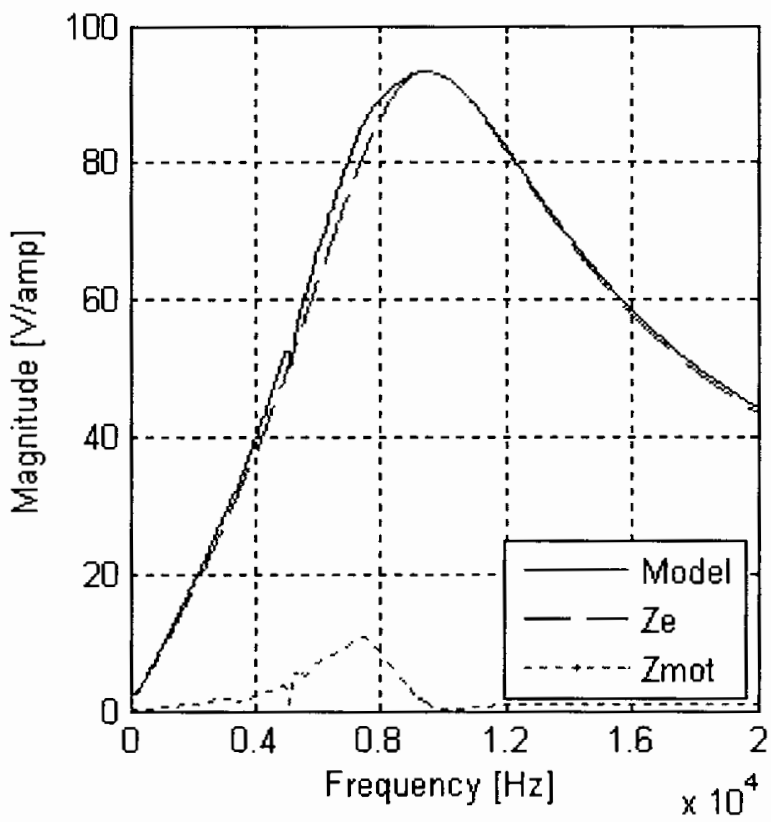

(b)

Figure 5.6: Comparison of model simulation and dynamic testing of the Terfenol-D transducer: (a) Zoomed magnitude response of $Z_{e e}$ and (b) magnitude response of the individual model components of $Z_{e e}, Z_{e}$ and $Z_{m o t}$. 


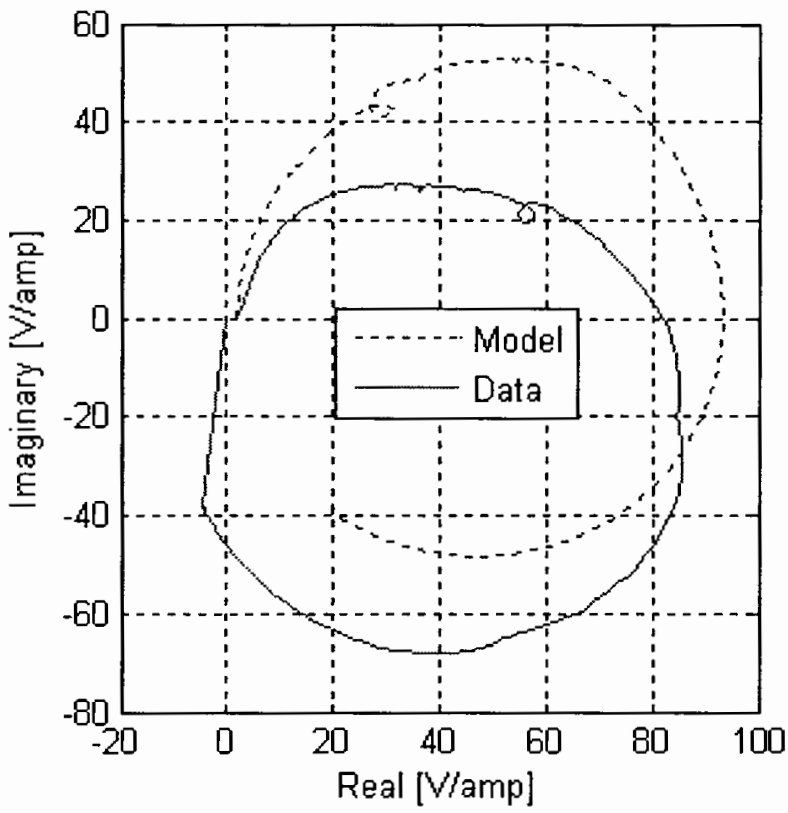

Figure 5.7: Nyquist plot of the electrical drive-point impedance for both experimental data and the dynamic system model.

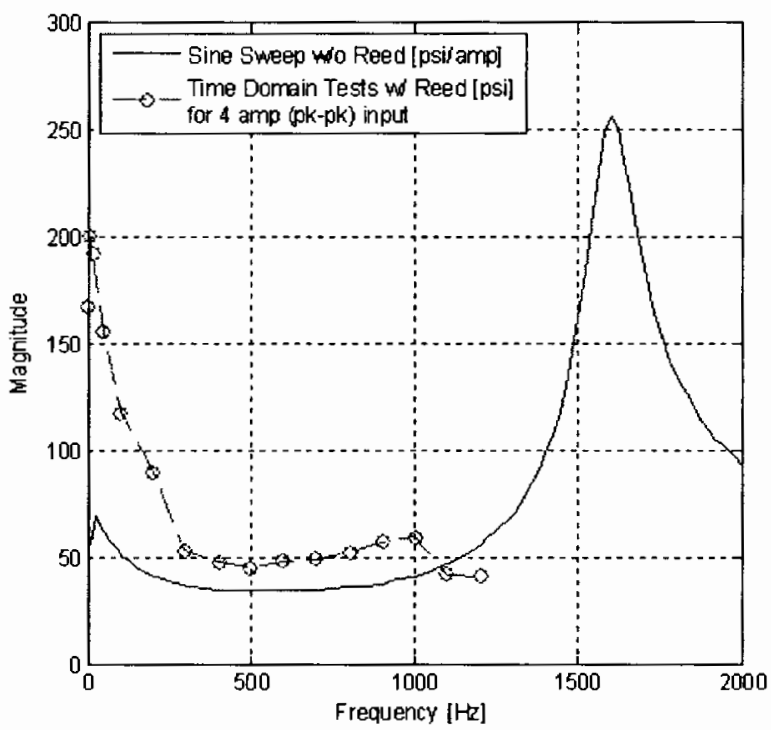

Figure 5.8: Harmonic blocked pressure testing alongside dynamic pressure data. Harmonic testing locates the frequency limitation of the reed valves at $1000 \mathrm{~Hz}$. 


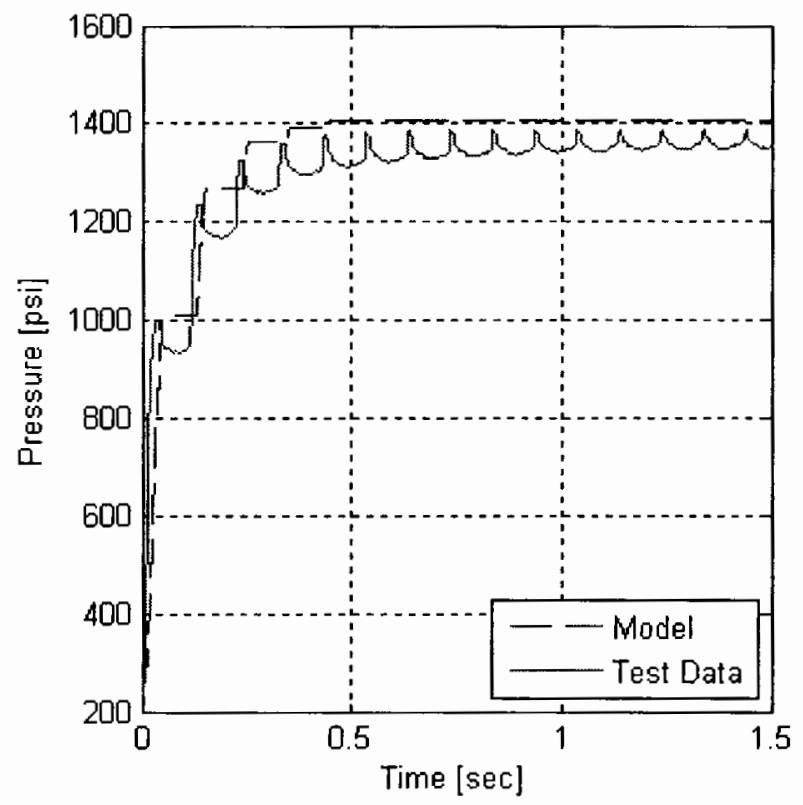

Figure 5.9: Pressure trace in the time domain for model simulation and harmonic test for a $10 \mathrm{~Hz} / 10 \mathrm{~A}$ sinusoidal input. 


\section{CHAPTER 6}

\section{CONCLUDING REMARKS}

\subsection{Summary}

In this research, an Electrohydrostatic Actuator was constructed, characterized and modeled. The EHA consists of a Terfenol-D pump (transducer) hydraulically connected to a linear actuator. The Terfenol-D transducer utilizes custom-made mechanical reed valves for rectification of the oscillatory pump flow creating highpressure unidirectional pump flow. Experimental work provides the characterization of the performance of the Terfenol-D pump and illustrates the electromechanical coupling of the Terfenol-D transducer. A linear dynamic system model is developed which couples the electrical, mechanical and fluid regimes of the pump; it predicts the maximum pressure of the Terfenol-D transducer; and predicts the location of the transducer resonance along with the dynamic behavior up to $20 \mathrm{kHz}$. The model identifies important characteristics of the pump that could not be known otherwise, such as the effective bulk modulus, Young's modulus, damping ratios and drive coil capacitance. The linear dynamic system model is an accurate representation of the Terfenol-D transducer considering the assumptions that were made in order to utilize linear modeling techniques for a highly nonlinear material. 
Three thickness of reed valves are tested and the thinnest, 0.005 in, yields the maximum Terfenol-D transducer performance. The 0.005 in reed valves have a frequency limitation of $1000 \mathrm{~Hz}$. A maximum blocked output pressure differential of 1100 psi is obtained at $10 \mathrm{~Hz}$ with a power consumption of $83.8 \mathrm{~W}$. The frequency limitation of the EHA occurs at $165 \mathrm{~Hz}$ and a maximum no-load velocity of $0.365 \mathrm{in} / \mathrm{s}$ is obtained at a frequency of $160 \mathrm{~Hz}$. The hydraulic cylinder used in the EHA introduces a large amount of nonuniform friction to the EHA, which severely limits its performance.

\subsection{Future Work}

One of the main goals in developing a smart material pump is to operate the pump at system resonance, the location of the largest electromechanical coupling of the transducer. The first step in progressing toward this end should be attempts to increase the bandwidth for the existing device with the existing passive mechanical check valves. Using a fluid of lower viscosity may reduce the fluid loading on the reed valves and allow for more volume flow. Also, since the thinnest reed valves produced the optimum performance of the Terfenol-D actuator, investigation of thinner reed valves may in turn increase the performance characteristics of the EHA. Studies have shown that the Young's modulus of Terfenol-D can be altered by changing the magnetic bias field through the sample [10]. By varying the magnetic bias through the Terfenol-D rod, the resonance of the Terfenol-D pump may be tuned to allow operation at a lower frequency.

In the next generation of fluid rectification, an analysis of the fluid dynamics of the pump head should be performed in order to minimize the turbulence around the reed valves and recirculation regions which are sources of energy loss in unidirectional 
pumps. The flow analysis should yield a geometry that best optimizes the flow characteristics of the pump head. This analysis could lead to other concepts that may help in the miniaturization of the pump such as implementing an array of tiny mechanical valves in order to increase the volume flow.

Active valves may also provide the ability to increase the the bandwidth of this device. Unimorph active valves [22] and magnetorheological fluid valves [27, 34] are two types of valves that were developed for this purpose and could be implemented into the Terfenol-D transducer design. Finally, the ultimate goal is to move a $4000 \mathrm{lb}$ 4 in at a velocity of $0.3 \mathrm{in} / \mathrm{s}$ and the key to succeeding in this goal is the means for rectification that of the oscillatory fluid flow. 


\section{APPENDIX A}

\section{SYSTEM PARAMETERS FOR DYNAMIC SYSTEM MODEL}

\begin{tabular}{|c||c|}
\hline Parameter & Value \\
\hline$k_{t}$ & $33.2 \mathrm{MN} / \mathrm{m}$ \\
\hline$E_{y}^{H}$ & $30 \mathrm{GPa}$ \\
\hline$q$ & $6.0 \times 10^{-9} \mathrm{~m} / \mathrm{amp}$ \\
\hline$k$ & 0.63 \\
\hline$\mu^{\sigma}$ & $2.17 \mu_{0}$ \\
\hline$\zeta_{t}$ & 0.09 \\
\hline$\zeta_{f}$ & 0.55 \\
\hline$\beta_{e f f}$ & $0.93 \mathrm{GPa}$ \\
\hline$b_{i}$ & $20.3 \mathrm{~N} \cdot \mathrm{s} / \mathrm{m}$ \\
\hline$b_{f}$ & $356 \mathrm{~N} \cdot \mathrm{s} / \mathrm{m}$ \\
\hline$k_{d}$ & $2.15 \mathrm{MN} / \mathrm{m}$ \\
\hline$k_{f}$ & $371 \mathrm{MN} / \mathrm{m}$ \\
\hline$L_{b l o c k}$ & $1.30 \mathrm{mH}$ \\
\hline$R$ & $2 \Omega$ \\
\hline$\alpha$ & $14.0 \mu \mathrm{s}$ \\
\hline$C$ & $208 \mathrm{nF}$ \\
\hline$n$ & $7525 \mathrm{turns} / \mathrm{m}$ \\
\hline Dia. & $12.5 \mathrm{~mm}$ \\
\hline$L_{t}$ & $114 \mathrm{~mm}$ \\
\hline
\end{tabular}

Table A.1: Mechanical, electrical and fluid parameters associated with the magnetostrictive pump used in the dynamic system model. 


\begin{tabular}{|c||c|}
\hline Fluid Parameter & Value \\
\hline \hline Bulk Modulus $\beta$ & $1.64 \mathrm{GPa}$ \\
\hline Dynamic Viscosity $\mu_{f}$ & $0.387 \mathrm{~Pa} \cdot \mathrm{sec}$ \\
\hline Density $\rho_{f}$ & $873 \mathrm{~kg} / \mathrm{m}^{3}$ \\
\hline
\end{tabular}

Table A.2: Hydraulic fluid parameters for Mobil DTE 20 series hydraulic oil used in this research. 


\section{APPENDIX B}

\section{TERFENOL-D TRANSDUCER ASSEMBLY AND PART DRAWINGS}

\section{B.1 Magnet Alignment}

Six Alnico magnets were purchased from McMaster-Carr and each has an OD of $1.5 \mathrm{in}$, an ID of 1.125 in and a length of $0.75 \mathrm{in}$. The magnets are cast and the IDs and ODs are not perfectly round. The ends of each piece are parallel. The alignment of these magnets is critical to the alignment of the pump head and base. If the magnets were not properly aligned, the force on the Terfenol-D would not be completely in its axial direction and would produce a bending moment on the Terfenol and eventually break it. The constraints on the magnet alignment are that the end magnets must be parallel with respect to each other, the IDs of the two end magnets must be concentric and the IDs of the two end magnets must be square with respect to the ends of the total magnet assembly. The following steps describe how the magnets were aligned:

- All six magnets are put on a custom made arbor and are pressed flush together by a bolt on the end of the arbor. Care must be taken that no scraps of metal are stuck between the magnets. Since the ends of each individual magnet are parallel, the ends of this assembly are parallel; 
- All magnets are ground to a common OD, making sure to remove as little material as possible;

- The ODs of the magnets are press-fit (0.0005 in) into an aluminum sleeve. The ODs are now square with the two ends;

- The IDs of the two end magnets are ground to $1.125 \mathrm{in}$. This dimension extends only 0.125 in, just enough so that there is no interference between from the cast ID with the matching lips on the adjustor cap and piston guide ring. The ground IDs are now concentric with each other and square with the two end faces.

\section{B.2 CAD Drawings and Additional Pictures}

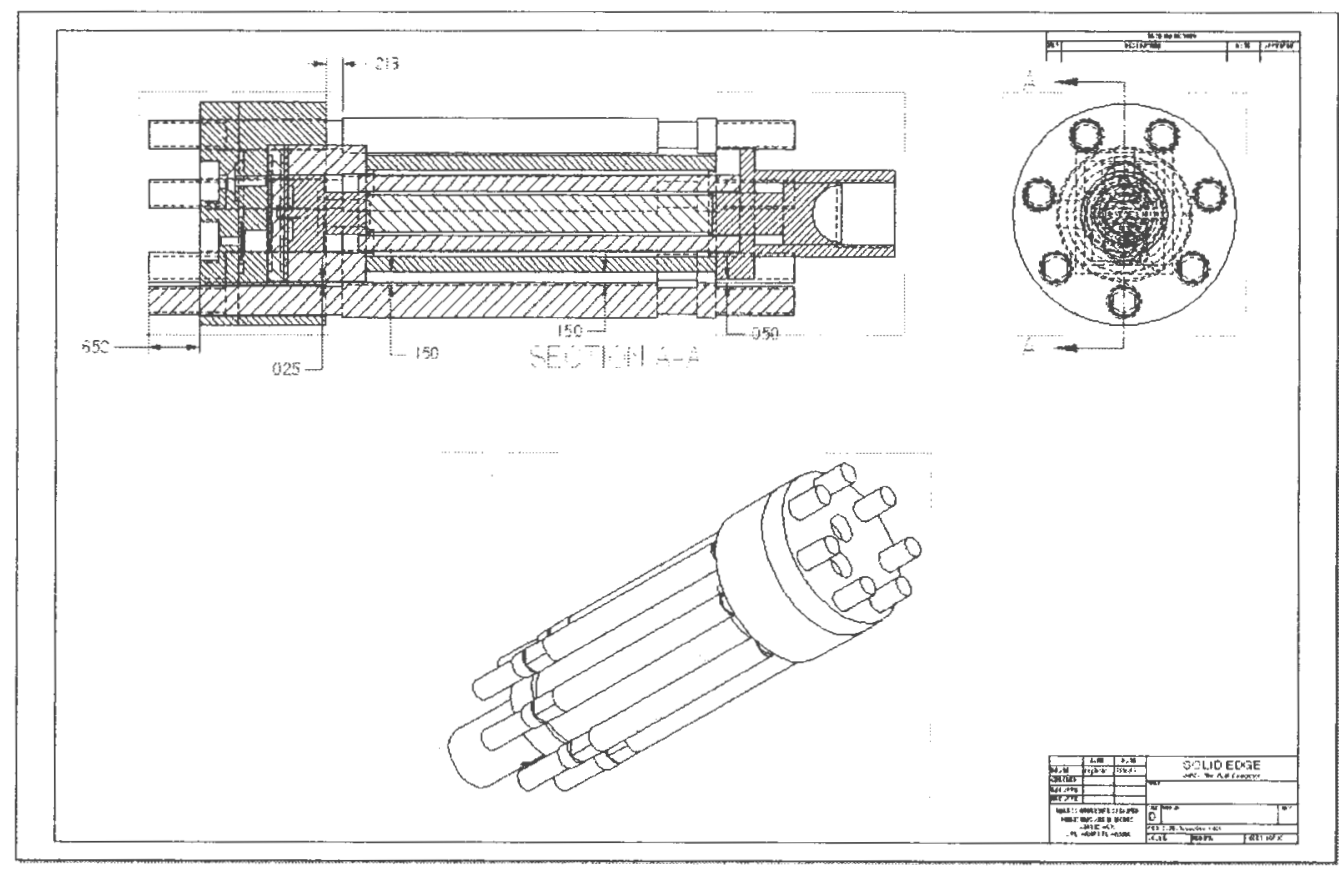

Figure B.1: Pump, total assembly except base. 


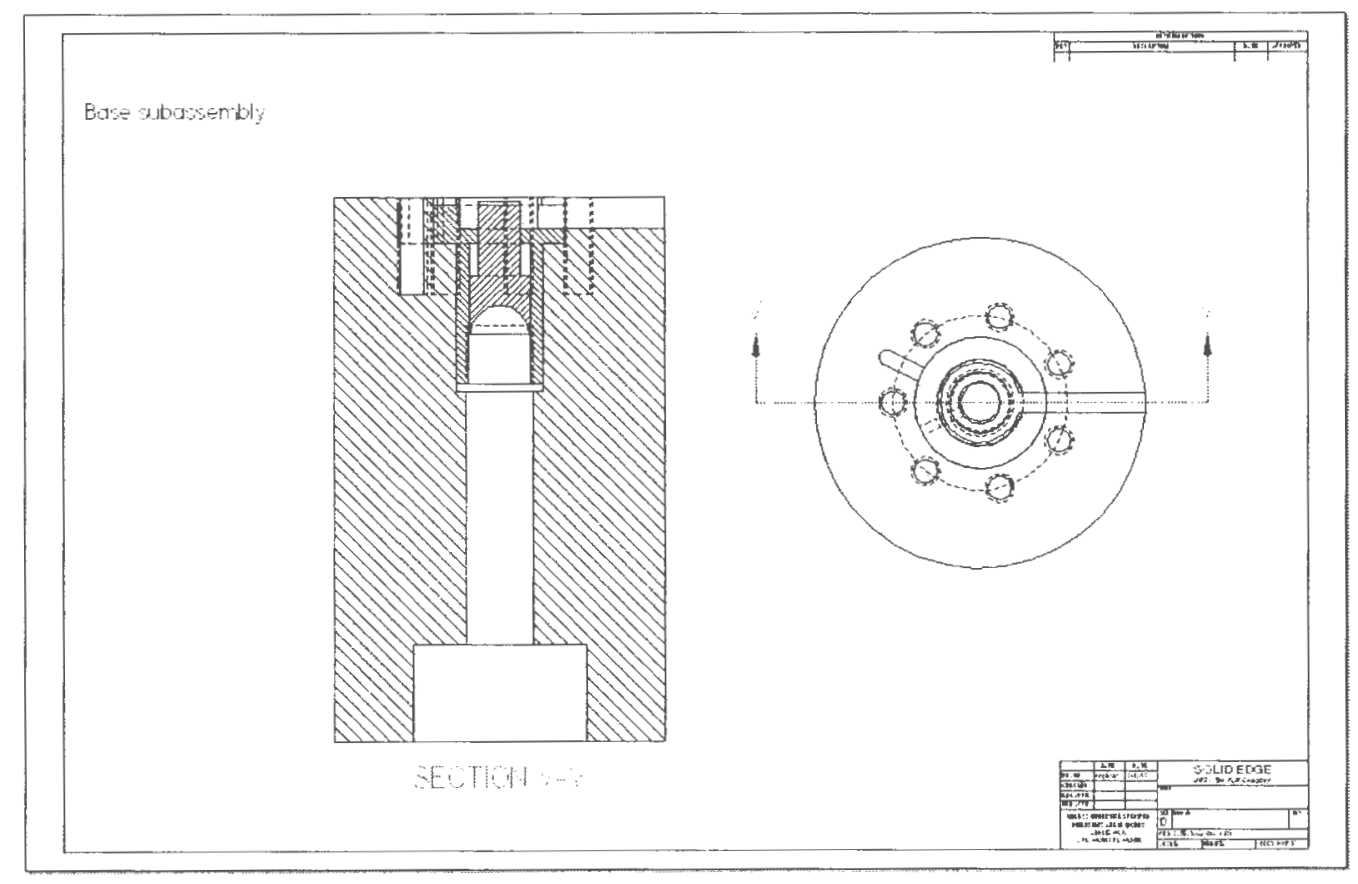

Figure B.2: Pump, base subassembly.

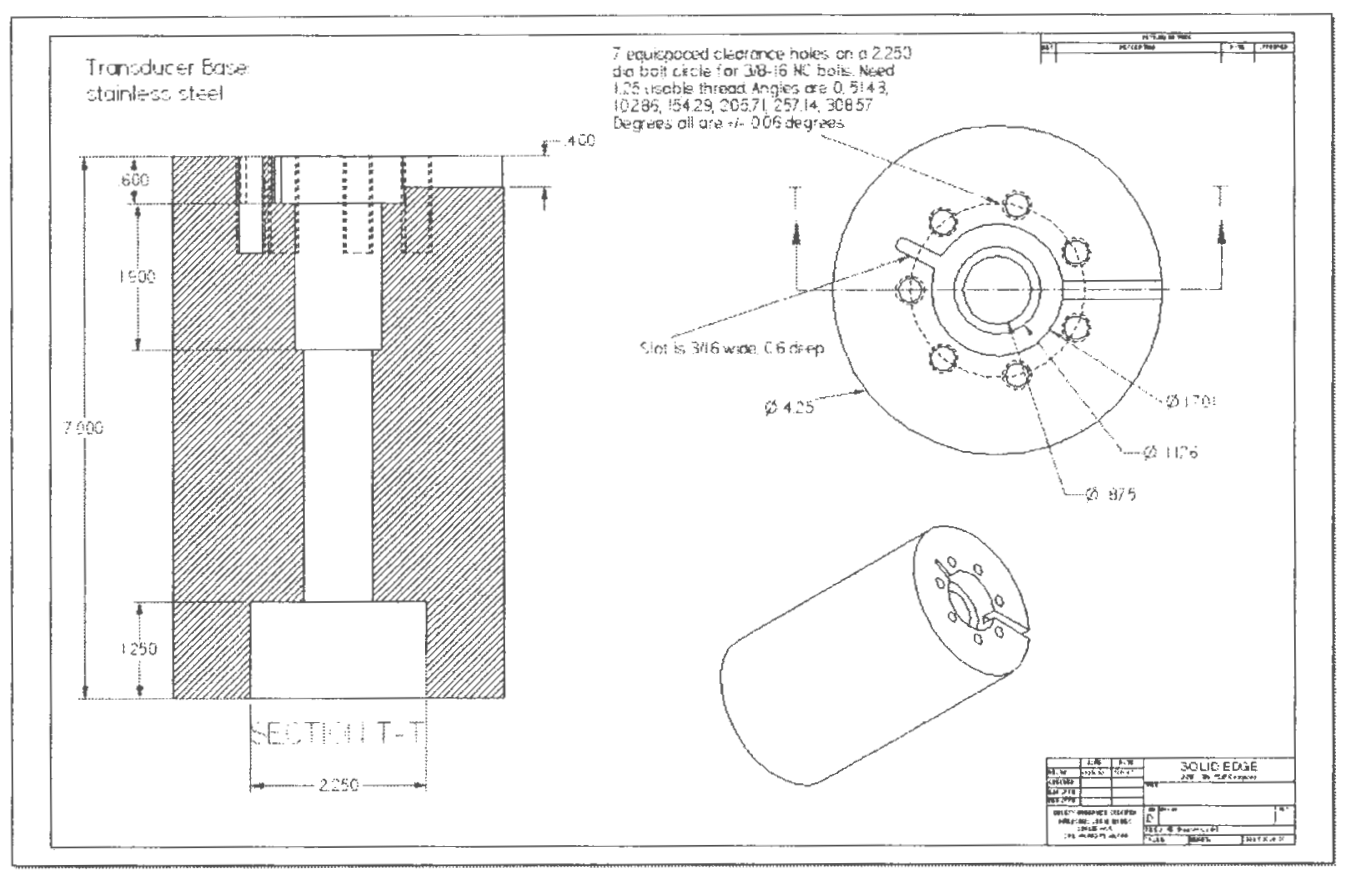

Figure B.3: Pump, base. 


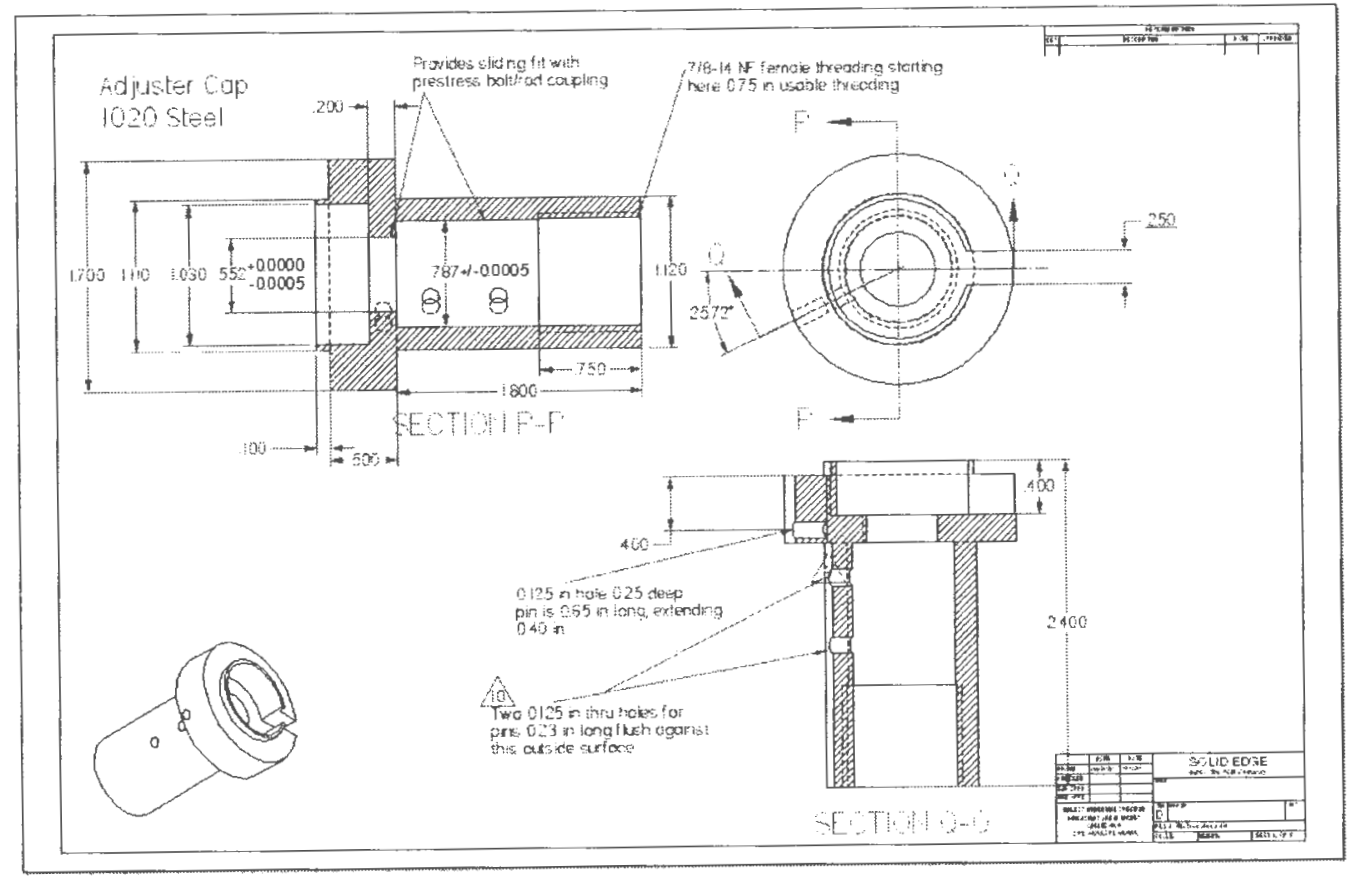

Figure B.4: Pump, adjustor cap.

Prestress boltRon Coupling

stress-firouf stepl1144

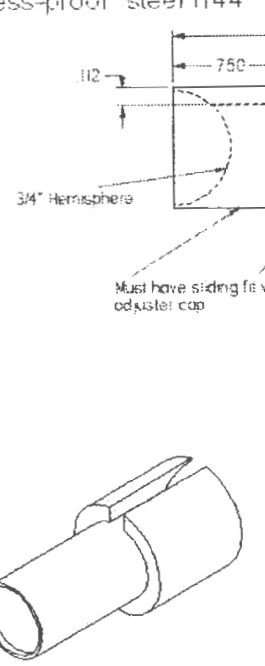

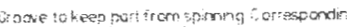

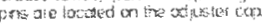

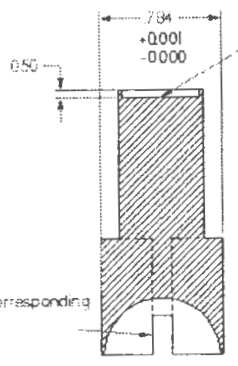

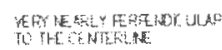

$$
\text { E } 10,6
$$

Figure B.5: Pump, Terfenol-D rod seat. 


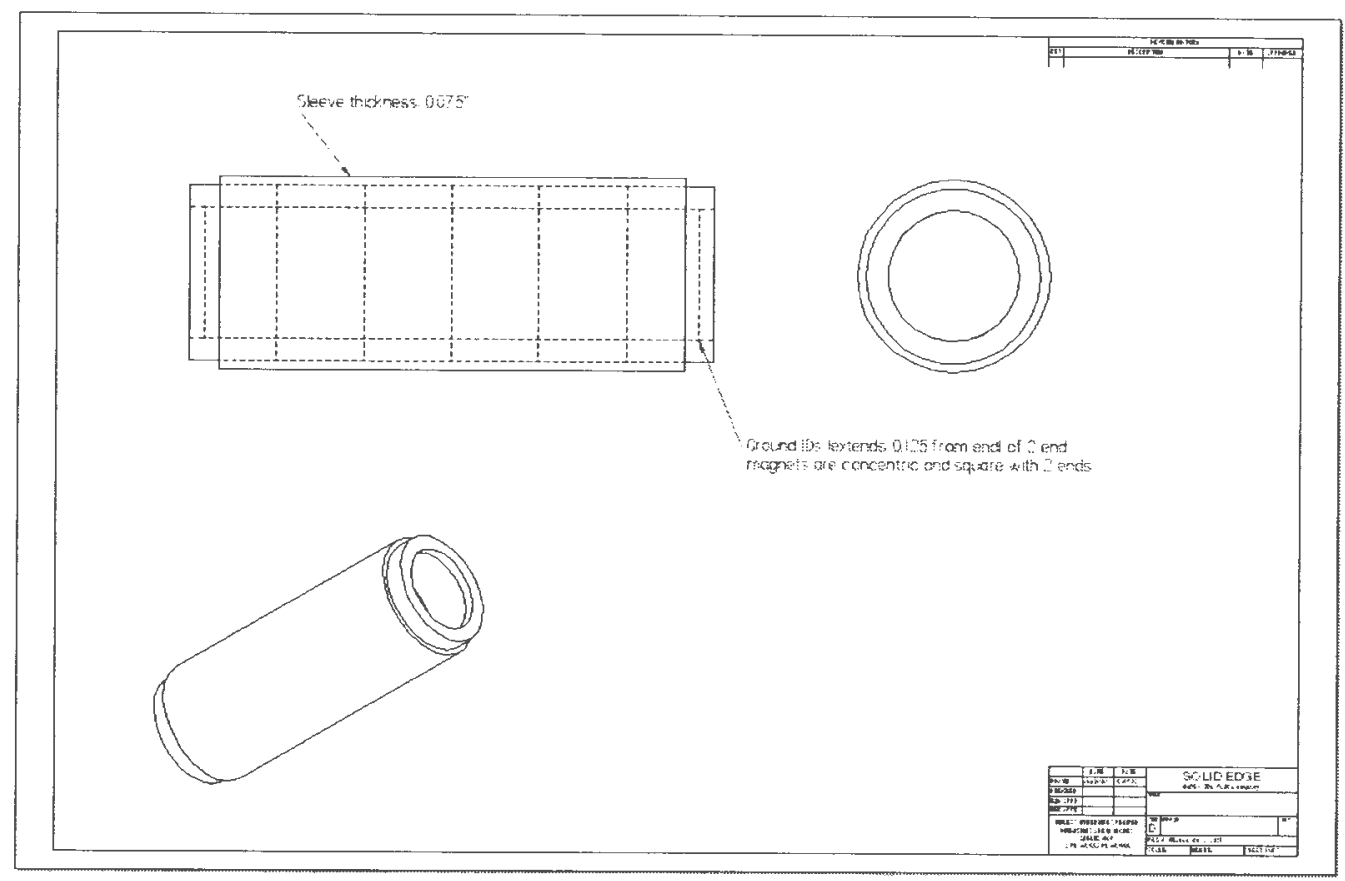

Figure B.6: Pump, magnet assembly.

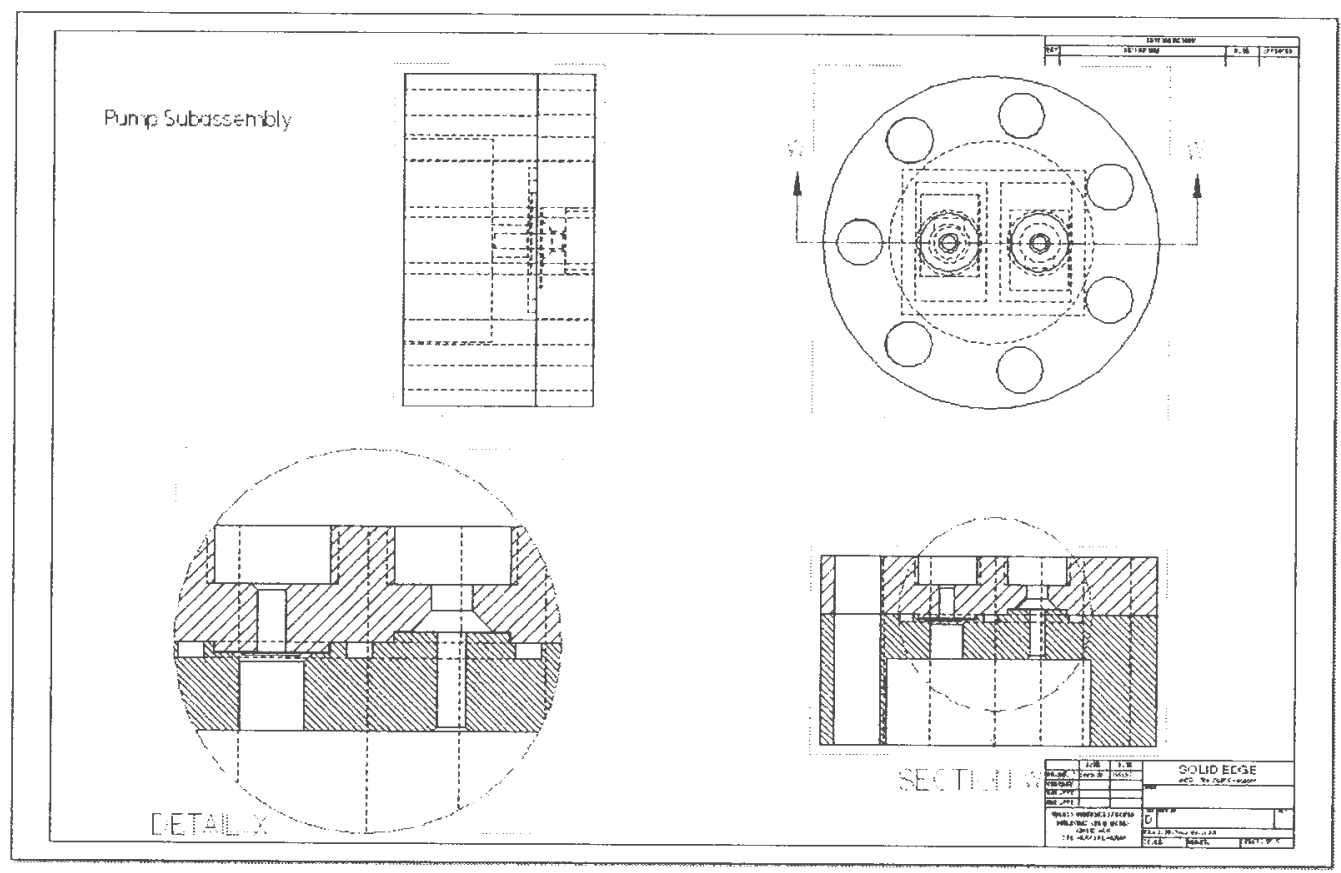

Figure B.7: Pump, head assembly. 


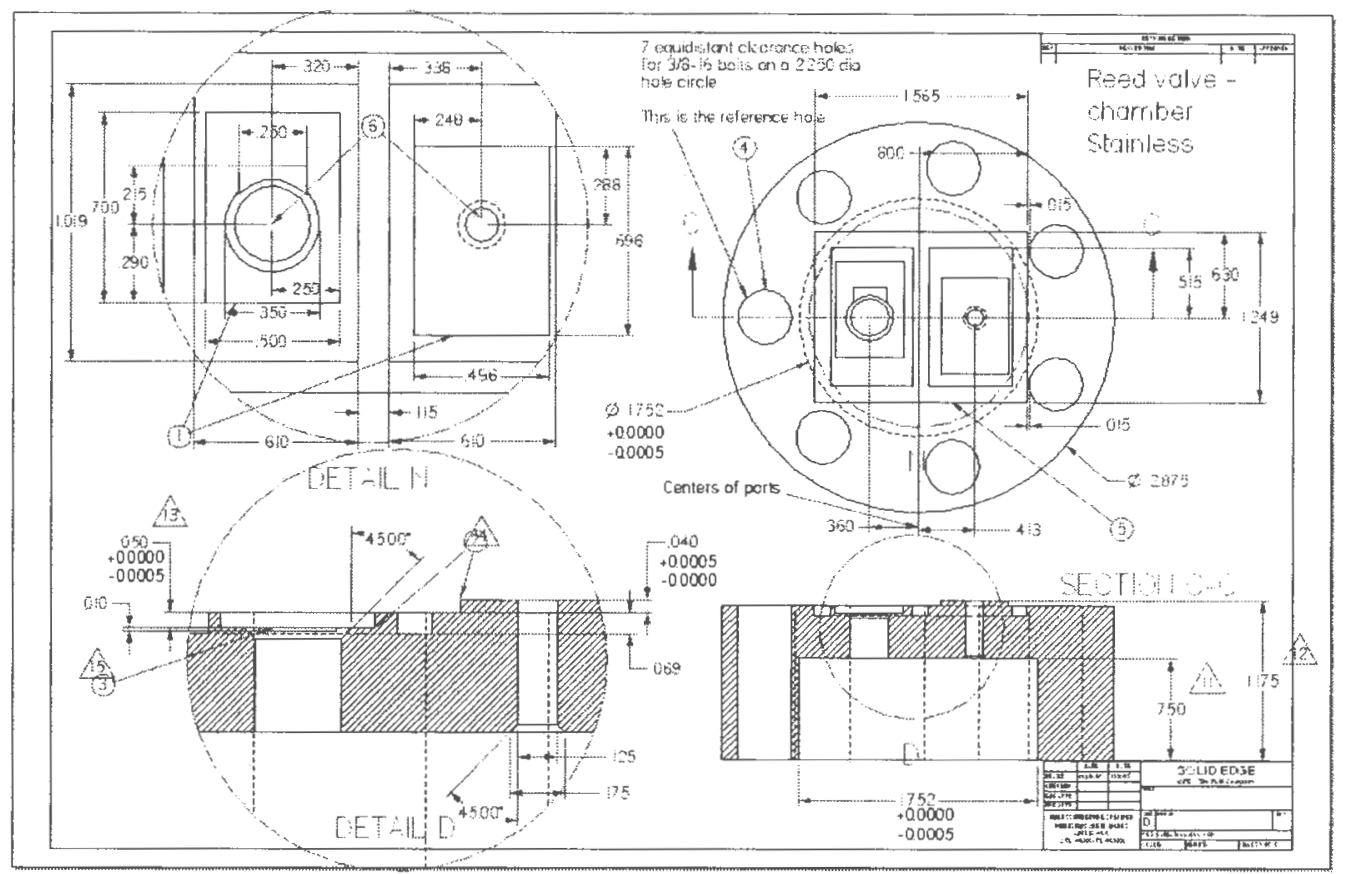

Figure B.8: Pump, chamber housing.

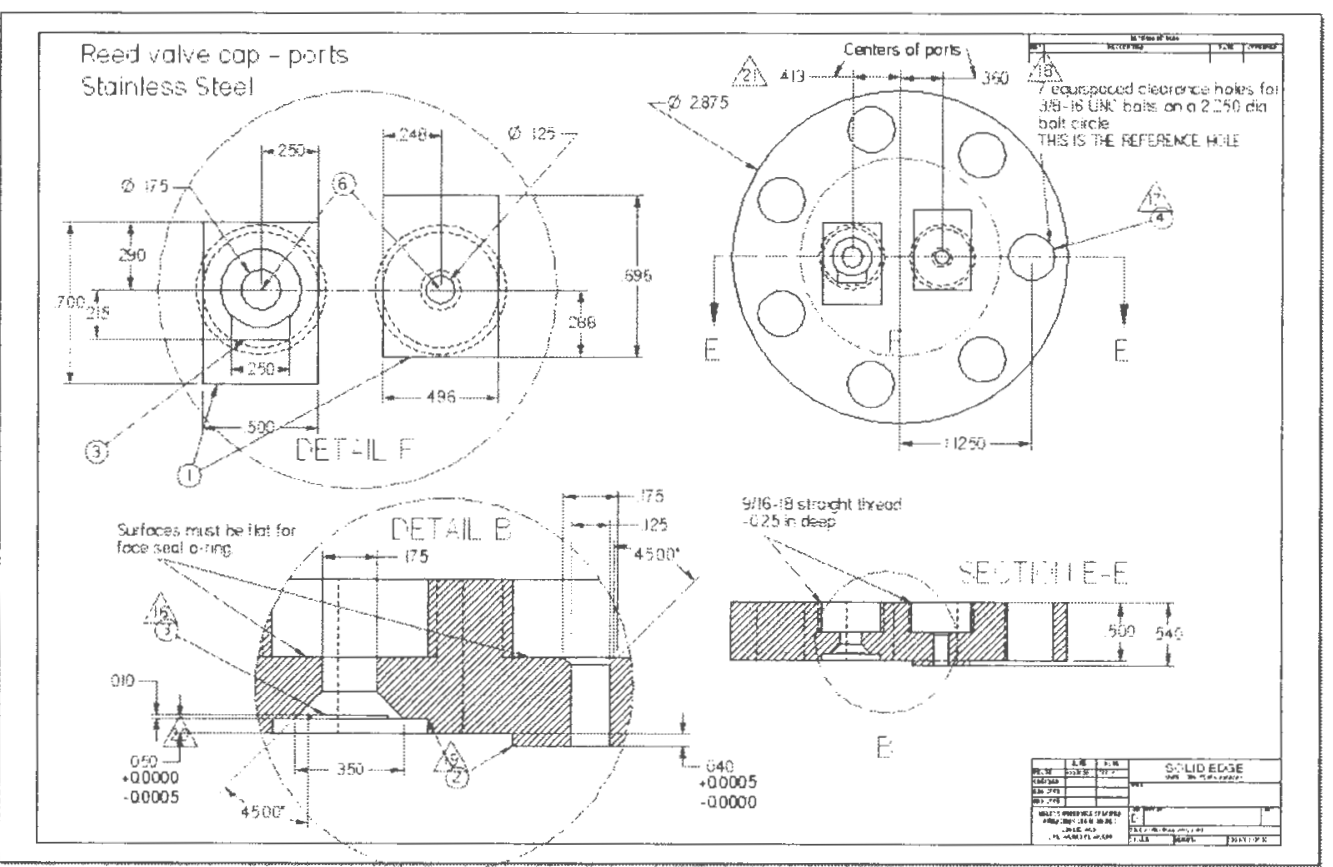

Figure B.9: Pump, port housing. 


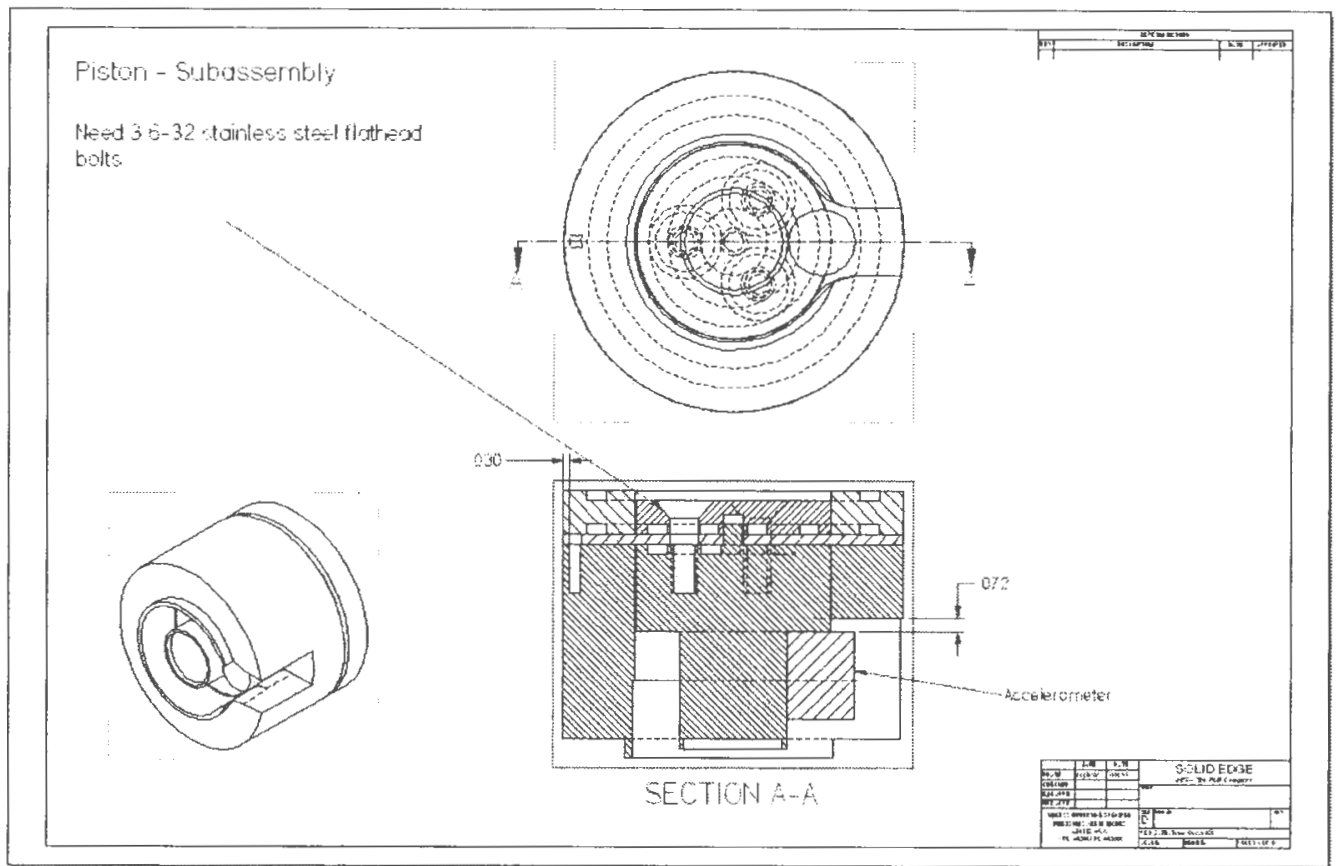

Figure B.10: Pump, piston subassembly.

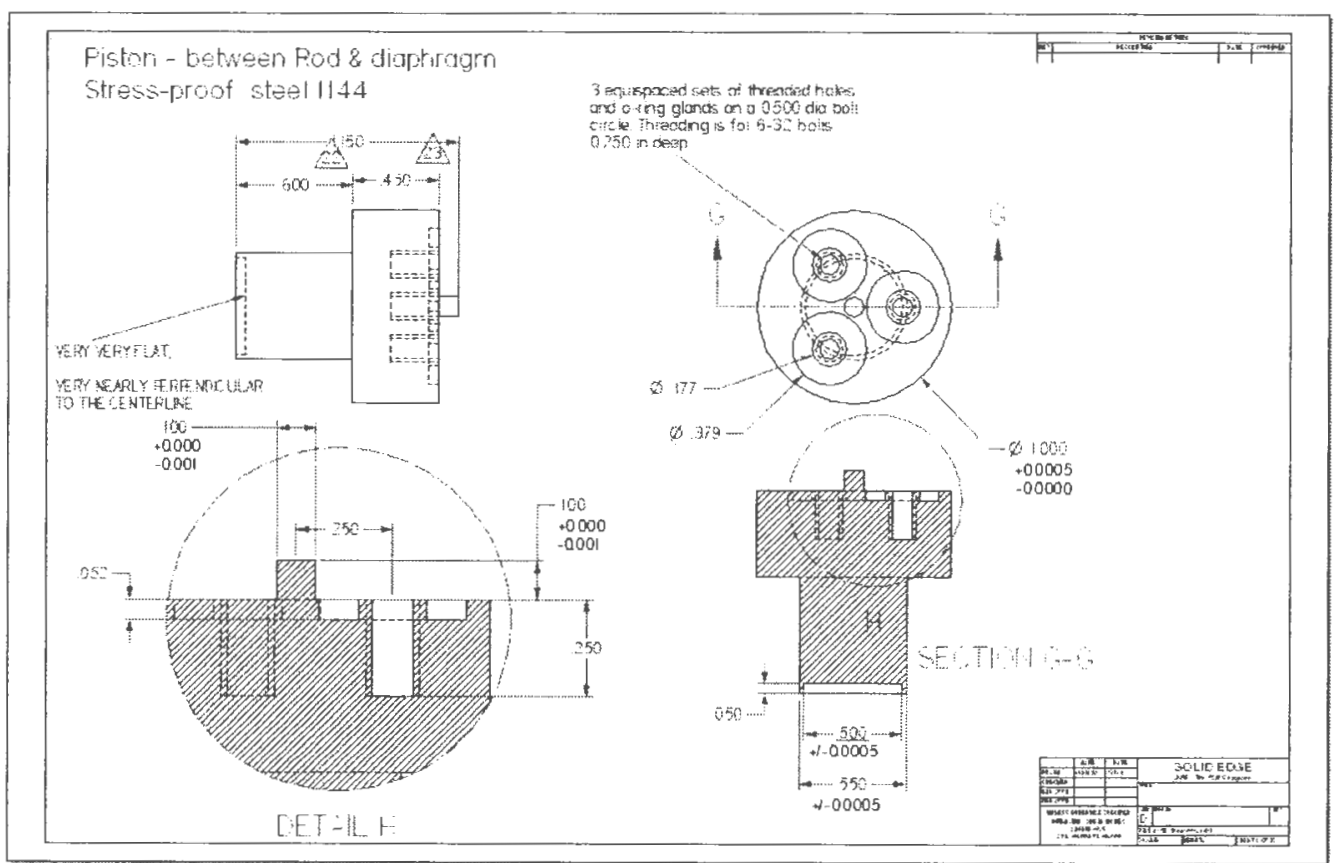

Figure B.11: Pump, piston in contact with Terfenol-D. 


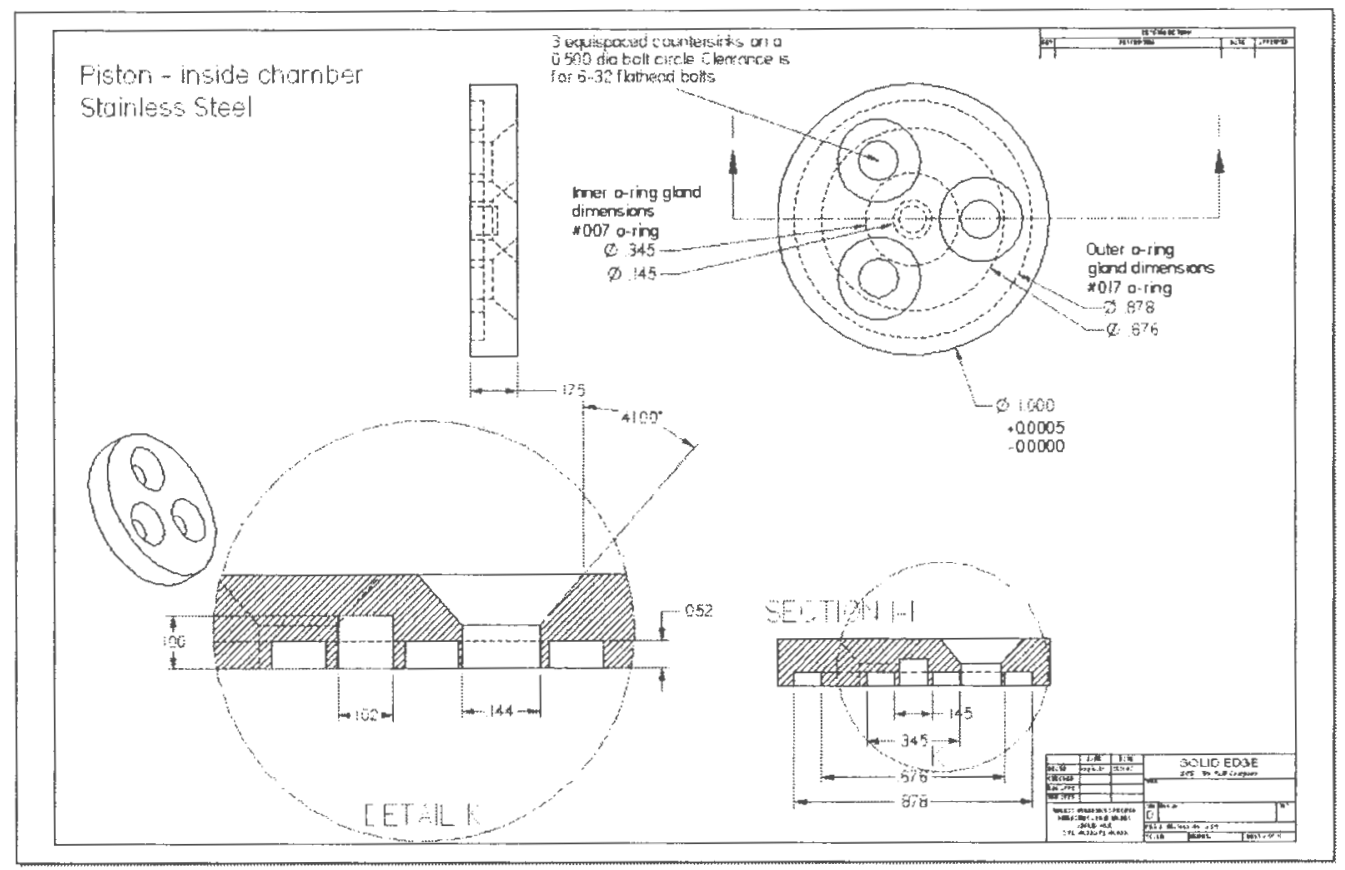

Figure B.12: Pump, top part of piston in contact with fluid. 


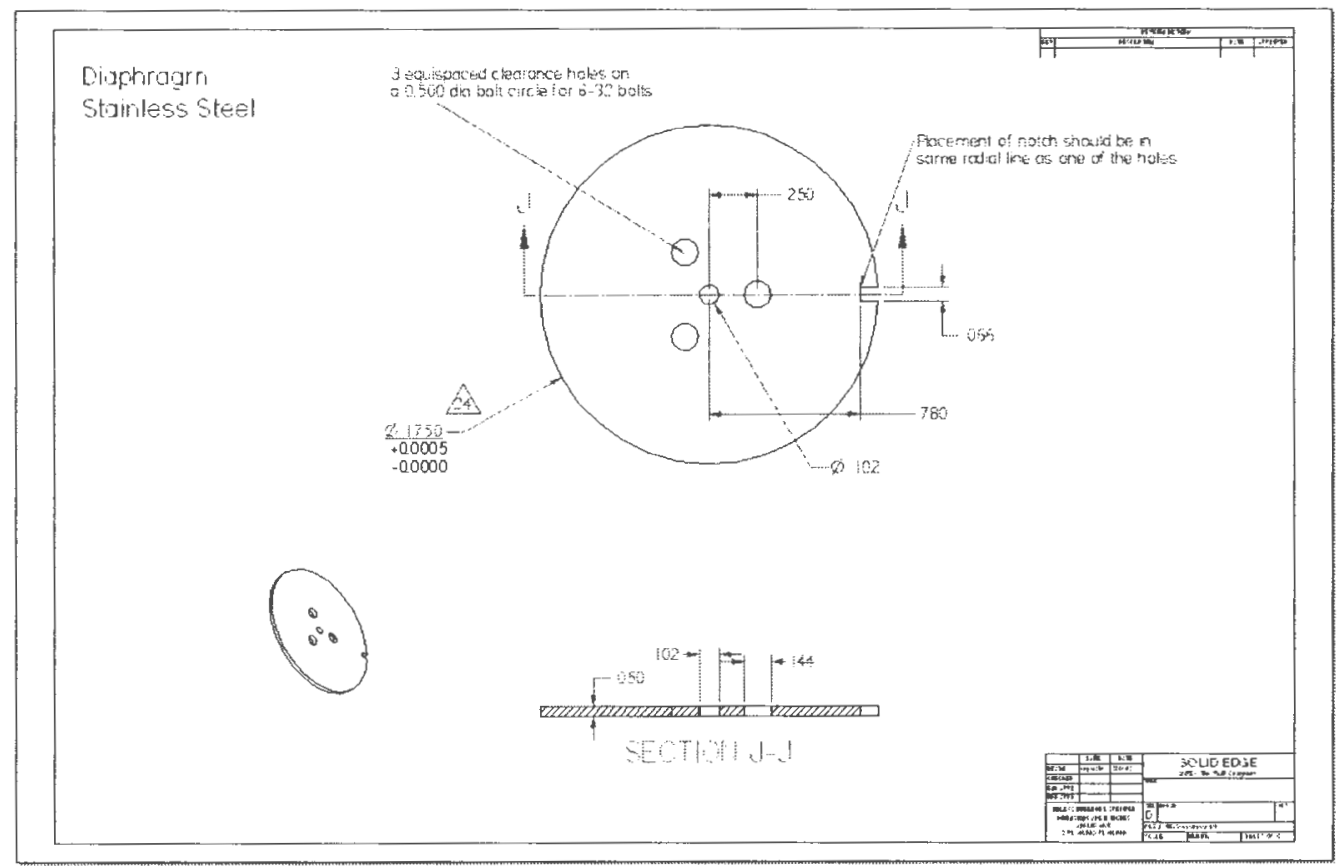

Figure B.13: Pump, diaphragm. Two were made. Thicknesses of 0.050 in and $0.005 \mathrm{in}$. 


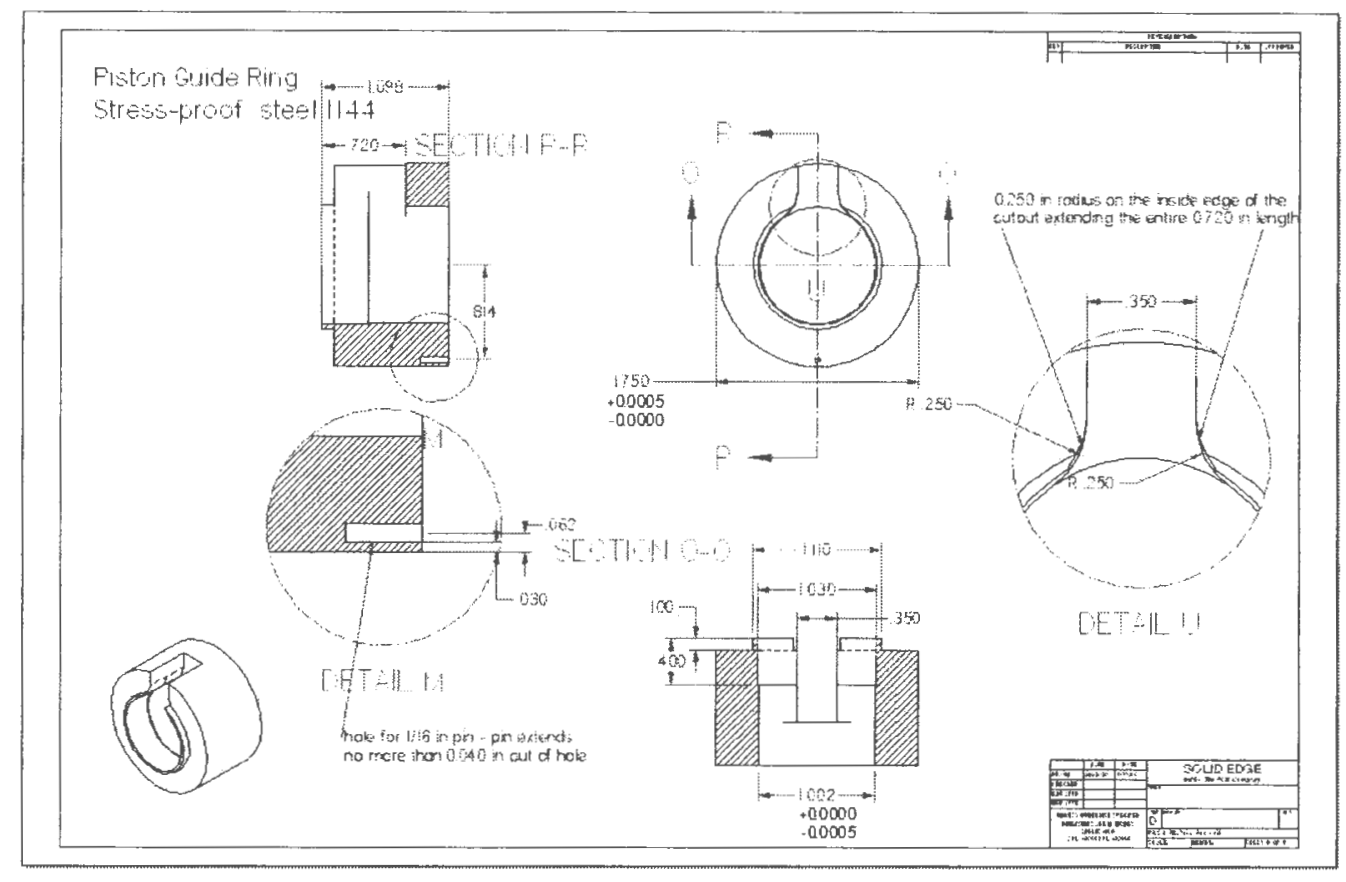

Figure B.14: Pump, guide ring.

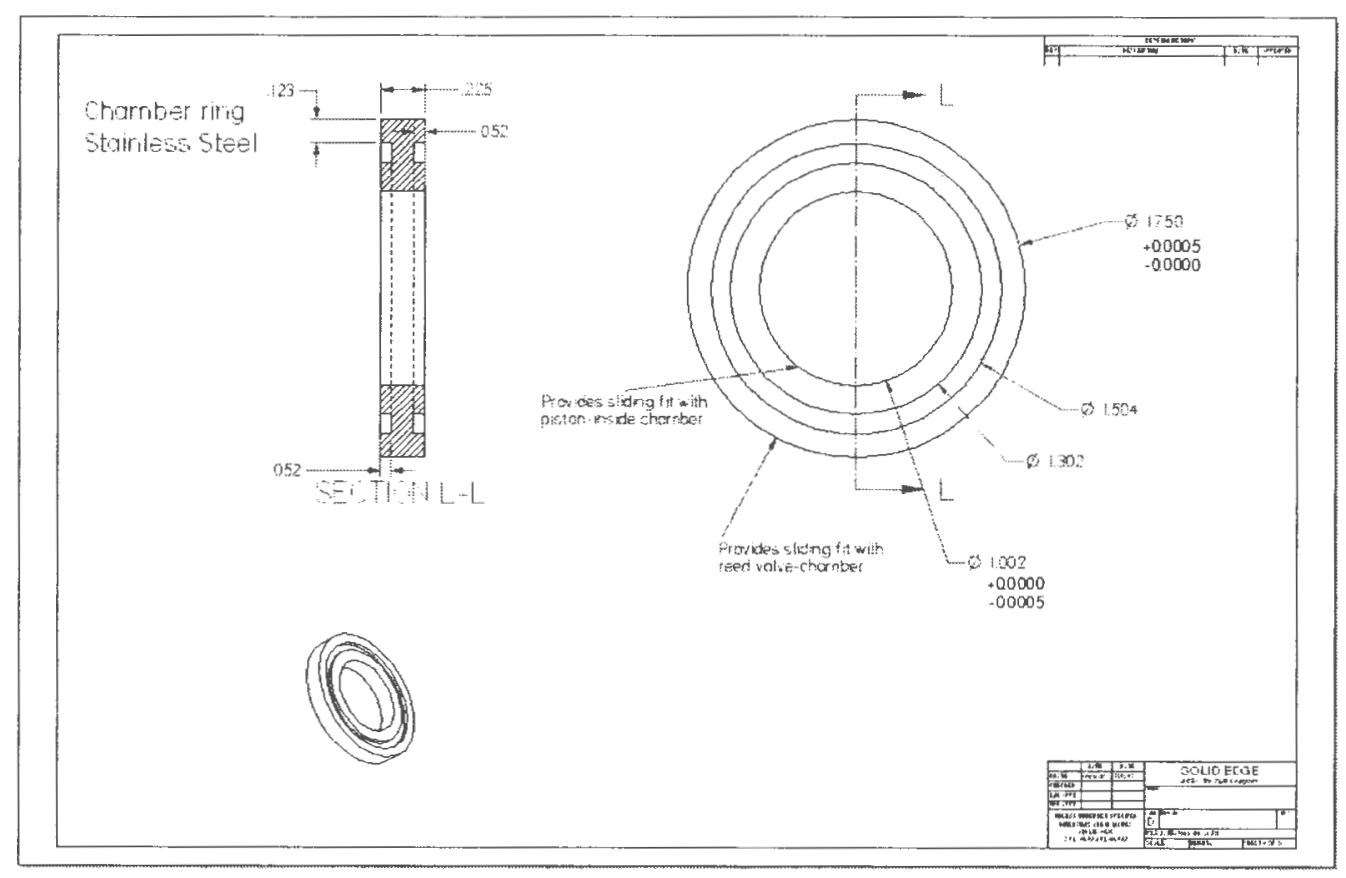

Figure B.15: Pump, chamber ring. 


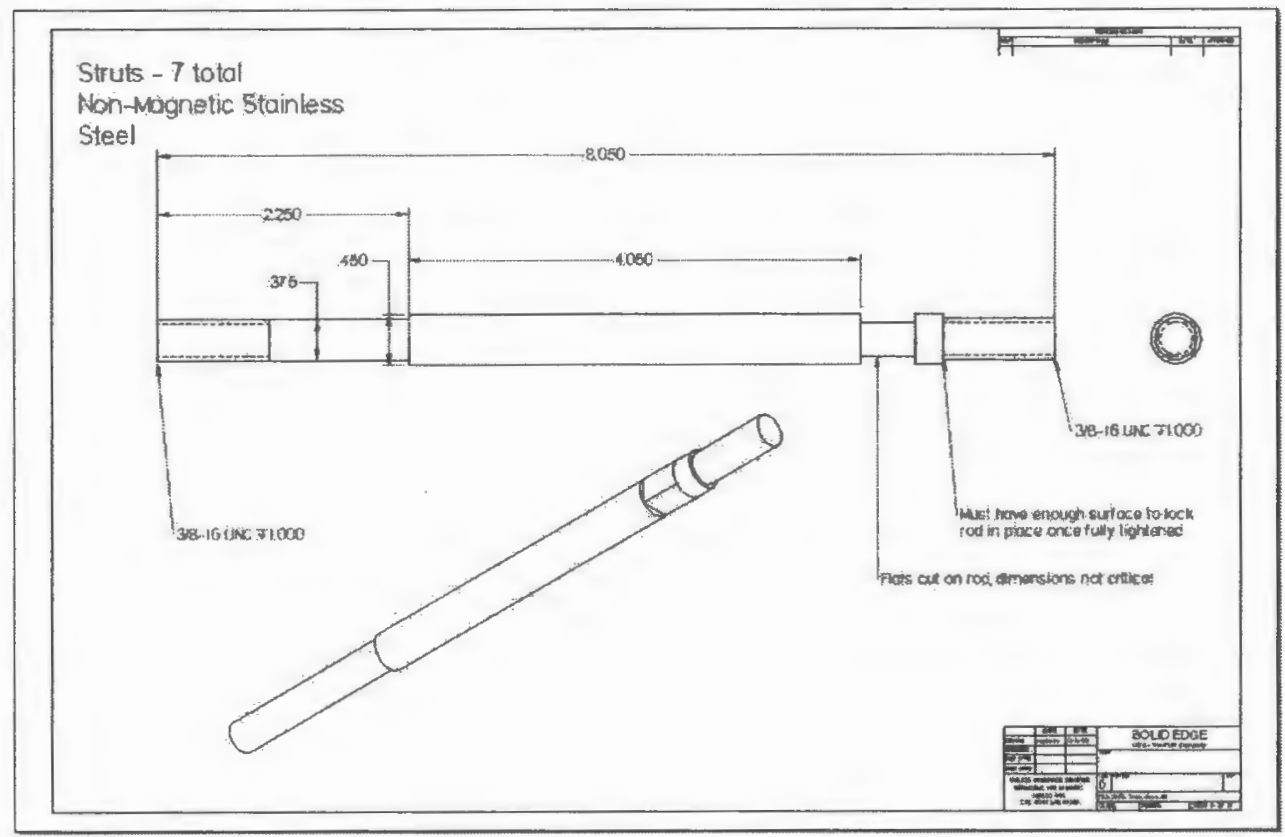

Figure B.16: Pump, strut.

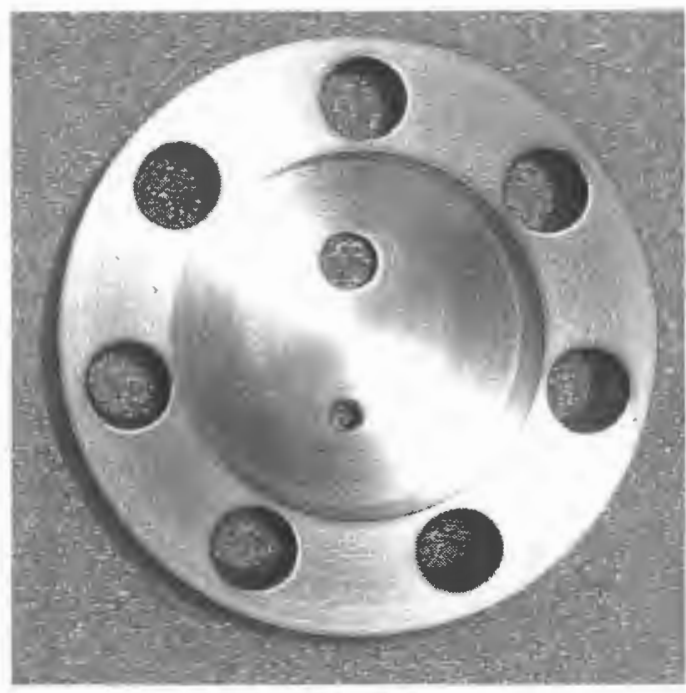

(a)

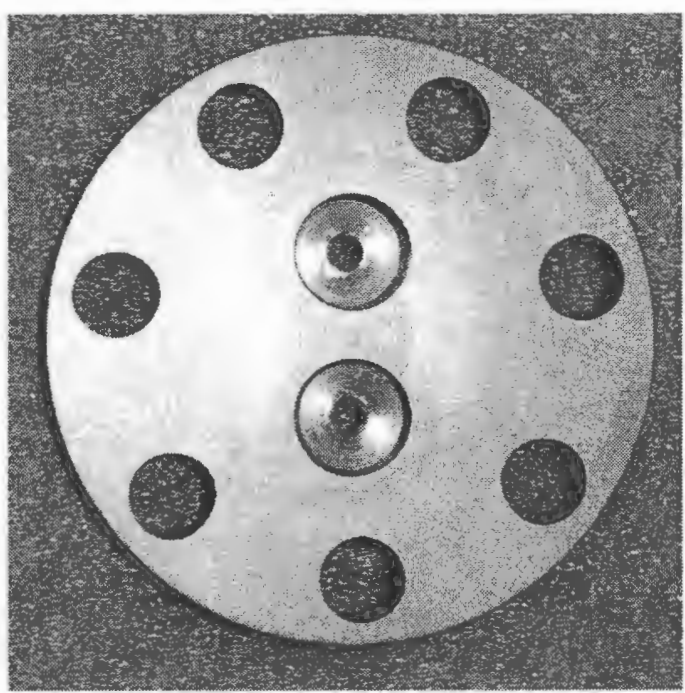

(b)

Figure B.17: Pump pictures: (a) pump head chamber housing and (b) ports in the port housing. 


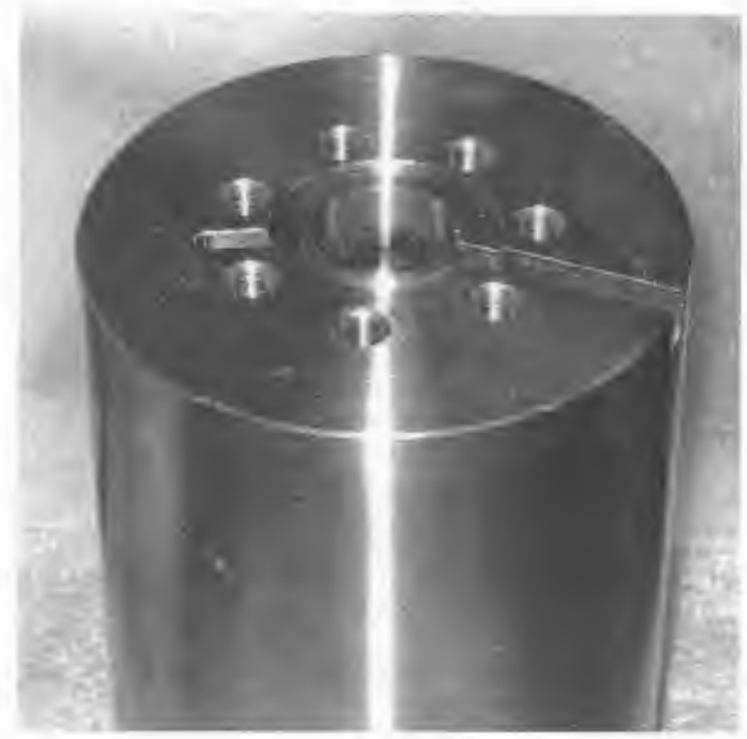

(a)

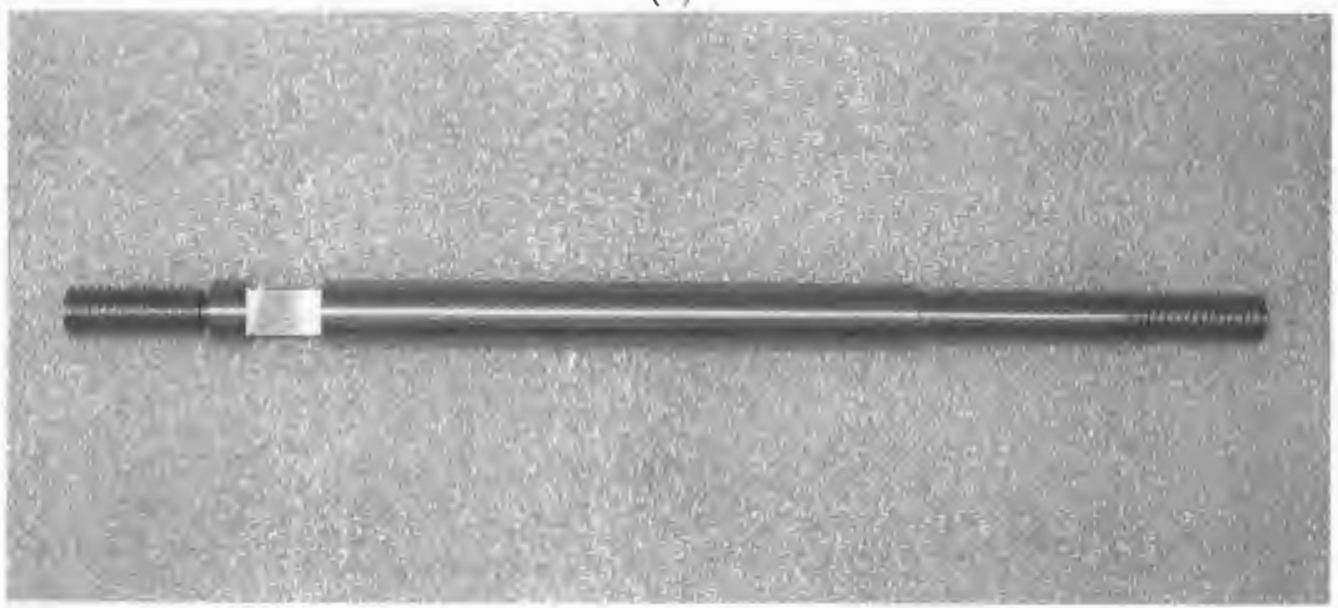

(b)

Figure B.18: Pictures of (a) the base subassembly and (d) one of the seven struts. 


\section{APPENDIX C}

\section{PRELIMINARY MAGNETOSTRICTIVE MOTOR AND PUMP}

The preliminary pump leading up to the final design consists of two separate components: (a) the Etrema Products vibratory Terfenol-D motor and (b) the pump head. The pump head is coupled to the motor through the piston. Sealing inside of the chamber is provided by a Parker \#2-228 dynamic O-ring. The pump head is a two chamber design (front and back) in which frequency rectification is to be performed by an external MR valve system (see Appendix $D$ for $M R$ valve discussion). The chamber and piston are both made out of aluminum to minimize weight of the pump. An aluminum holding block clamps the pump head and Terfenol-D motor together. The pump is first tested with hydraulic oil for characterization. Characterization begins with the Terfenol-D motor followed by blocked output measurements and issues with the pump design.

\section{C.1 Terfenol-D Motor Unloaded Resonance}

A 2.5 in diameter aluminum piston is attached to the Terfenol-D motor and a stepped sine frequency sinusoid is input to the device to locate the resonance. The stepped sine tests are controlled by Data Physics SignalCalc ACE Dynamic Signal 


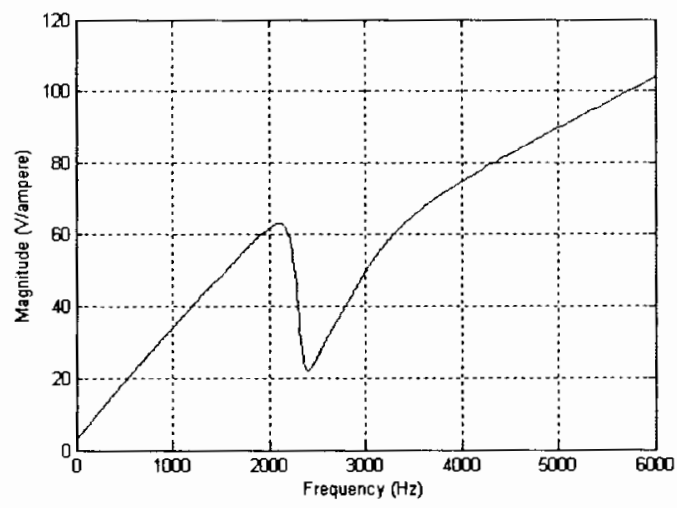

(a)

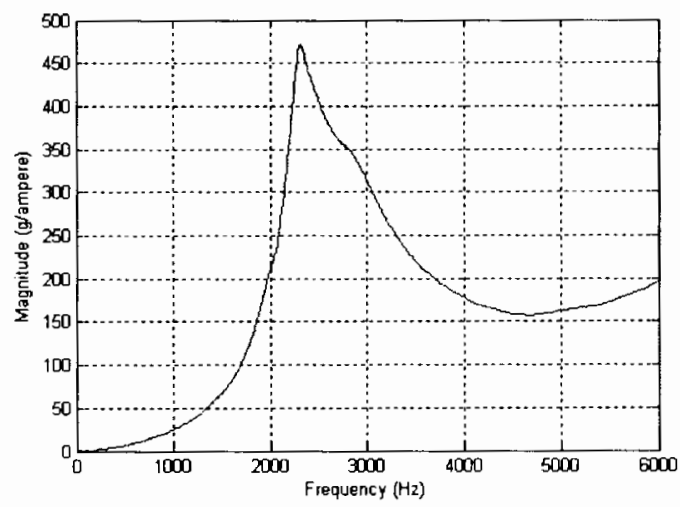

(c)

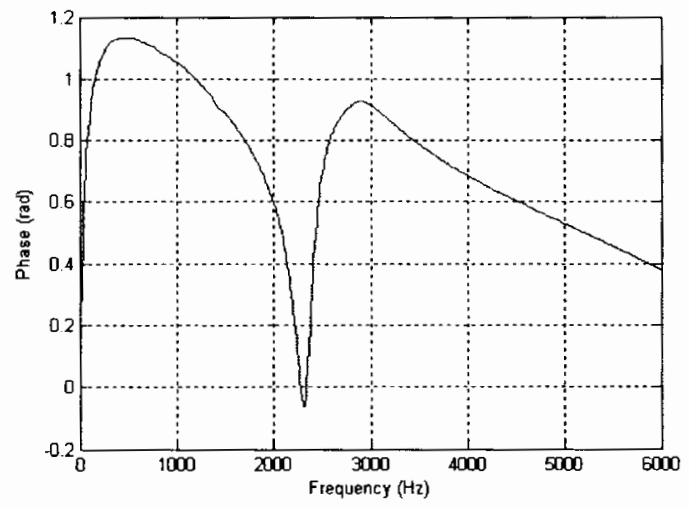

(b)

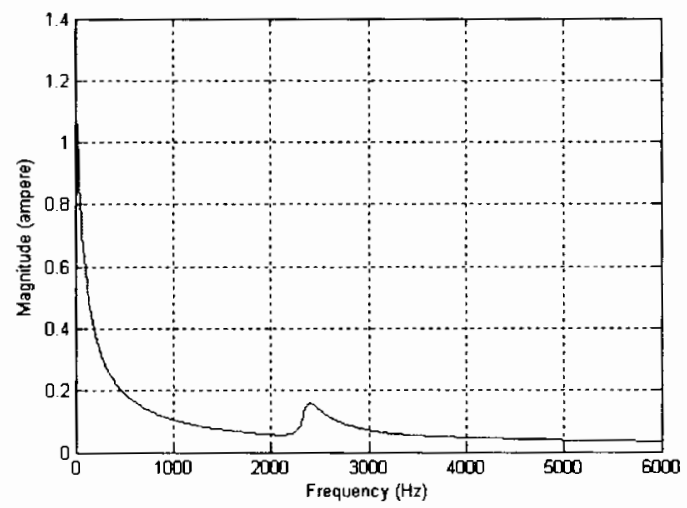

(d)

Figure C.1: Frequency (a) and Phase (b) response for impedance transfer function, the acceleration/input current of the piston (c) and the magnitude of the input current spectral density (d).

Analyzer software and Quattro hardware which record the input current, voltage, and piston acceleration. Resonance occurs at the peak in the acceleration/input current frequency response, Fig. C.1(c). For the Terfenol-D motor loaded with the 2.5 in diameter piston, mechanical resonance is located at $2320 \mathrm{~Hz}$ with a magnitude of $472 \mathrm{~g} / \mathrm{amp}$. A decrease in resonance is expected once the motor is loaded by the fluid and pump. 


\section{C.2 Blocked Output Measurements}

Measurements were conducted on the Terfenol-D pump under blocked output conditions. The pressures created by the magnetostrictive pump with its ports blocked are the theoretical maximum pressures that the pump is capable of producing. Theoretically the pump should produce the maximum pressures at or near the loaded pump resonance.

Some of the issues with this pump that will be discussed hereon are friction/resistance, air bubbles, alignment, and energy dissipation. The experiment is setup so that initially only the front chamber is measured for blocked pressures. In trying to simultaneously measure the blocked pressures of both ports, the pump will create a vacuum and work against itself. For every extension of the Terfenol-D transducer, pressure would be created in the front chamber while a vacuum would exist in the back chamber, thus putting extra loading onto the transducer. Pump design and analysis has shown that vortex recirculation rings form in the discharge tube during discharge and the pumping chamber during intake [30]. A chamfer with an $\mathrm{r} / \mathrm{d}$ ratio of 0.5 has been shown minimize the recirculation regions during pump flow. Thus, a chamfer was machined to create better flow characteristics out of the chamber. This along with a stiff pumping chamber is expected to increase the pump performance.

Stepped sine measurements are conducted under blocked output conditions and controlled by the Data Physics system. The input current, voltage, piston acceleration, and chamber pressure are recorded and a schematic of the test setup is shown in Fig. C.3. The frequency response of the acceleration per input current and chamber pressure per input current for tests taken on subsequent days are shown in Fig C.2. 


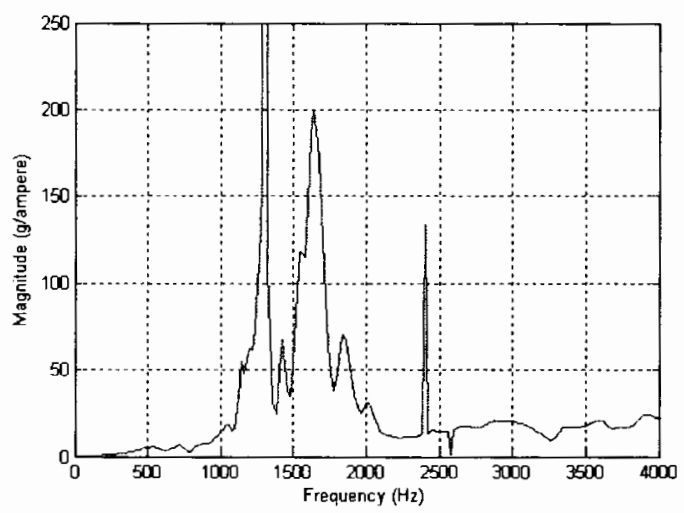

(a)

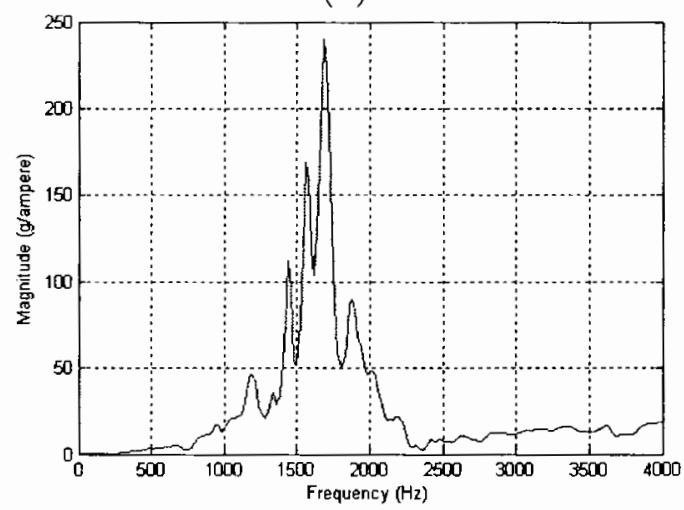

(c)

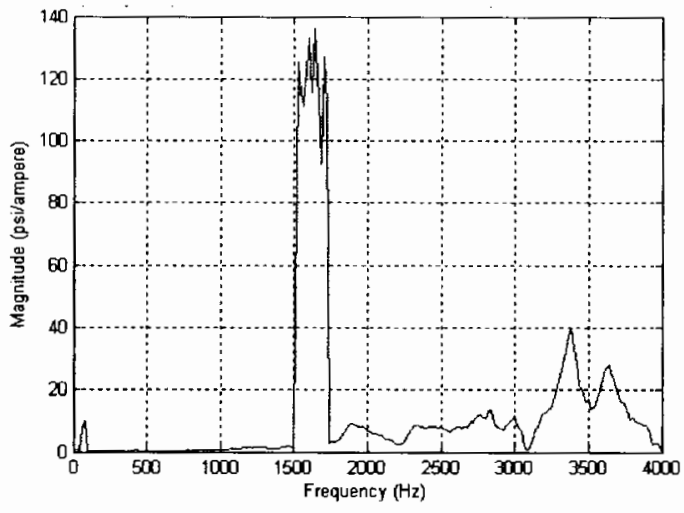

(b)

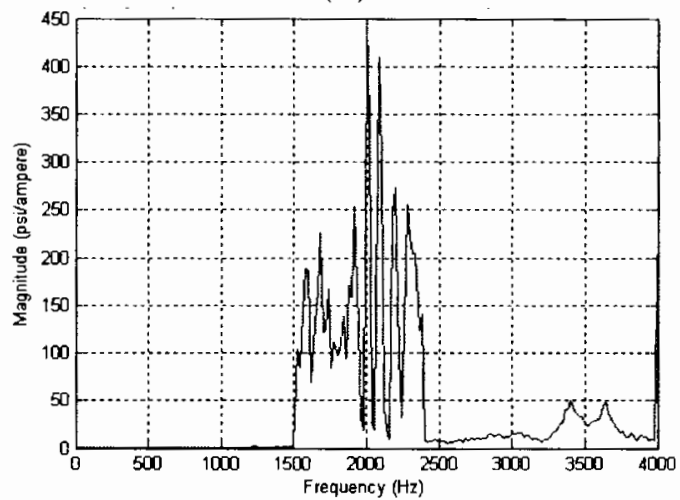

(d)

Figure C.2: Frequency response for acceleration/input current of the piston (a) and (c) and chamber pressure/input current (b) and (d).

Two sets of data are given to illustrate the changes that resulted from a slight modification of the aluminum block that clamps the transducer and pump. The pressure sensor used in these experiments is a diaphragm type pressure sensor from OMEGA. It has a response time of $1 \mathrm{msec}$ which means that it should give accurate pressure readings up to $500-1000 \mathrm{~Hz}$. The only pressure output recorded occurs after $1000 \mathrm{~Hz}$. Even though we measured approximately $130 \mathrm{psi} / \mathrm{amp}$ before the modification and $150 \mathrm{psi} / \mathrm{amp}$ after the modification, the data is questionable because 
it is past the bandwidth $(1-1000 \mathrm{~Hz})$ of the sensor. Also, this OMEGA pressure sensor has a very small capillary opening that leads to the diaphragm. It is believed that the air is being trapped inside this capillary tube during these measurements. The current method of clamping the system together is unsatisfactory and allows movement of the chamber housing.

Before the next set of tests are conducted, major modifications are done to the holding block, a SENSOTEC pressure sensor replaces the OMEGA sensor, and different ways of removing the air are implemented. The SENSOTEC pressure sensor is a diaphragm sensor but does not have a capillary tube as the OMEGA sensor does. There is just a threaded hole (1/4-18 NPT) that leads directly to the diaphragm. The air can be easily removed from this sensor. The SENSOTEC sensor has a larger bandwidth, up to $2000 \mathrm{~Hz}$, allowing us to examine a broader frequency range. Upon switching pressure sensors, a jump in pressure readout of approximately 20 psi was recorded for an input sinusoid of $10 \mathrm{~Hz}$ supporting the assumption that air was in the capillary tube of the OMEGA pressure sensor.

During pump operation the presence of air is a severe hinderance to the hydraulic circuit. Any air trapped in the oil will effectively kill the bulk modulus of the oil and thus the efficiency of the pump. Ways of dealing with this problem include bleeding the system of any air and creating a vacuum on the hydraulic oil. Applying pressure to an open hydraulic circuit will force the trapped air out of a bleed port, usually placed at the highest point in the circuit. However, the air cannot be removed from the current pump design in this manner. The pump chamber has only one port on each side. When pressure is applied to the system, the air trapped in the chamber will compress and remain in the system. This method neither removes the entrained gases 


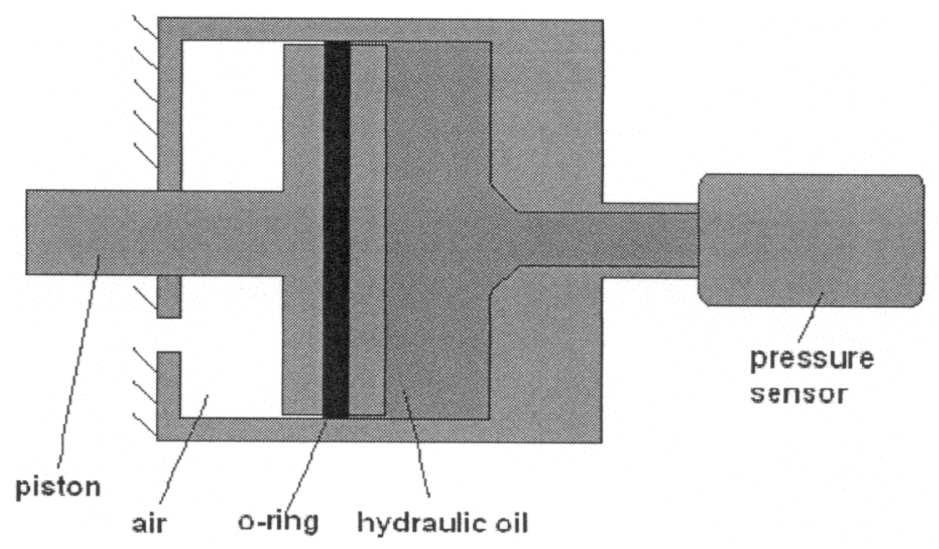

Figure C.3: Experimental diagram for the blocked chamber measurements. The front chamber is filled with hydraulic fluid and blocked, while the back chamber is left open. A pressure sensor measures the pressure created by the pump.

that are present in the oil. Applying a vacuum to the oil removes any air trapped in the system and the entrained gases present in the oil itself.

A vacuum pump capable of pulling $29 \mathrm{mmHg}$ was purchased and used to rid the hydraulic circuit of any trapped air and entrained gases. Once the vacuum is applied, small bubbles are seen rising from the oil. After the oil is degassed, the system is returned to atmospheric pressure and a manual valve is closed that blocks the front chamber, while the back chamber is left unfilled and open as illustrated in Fig. C.3.

The modified system holding block, Figs. ??-??, is made of two pieces that clamp the Terfenol-D transducer and pump housing separately. The sliding design of the blocks allows the user to more easily determine the chamber height. A small aluminum block with known length is clamped in between the two sliding pieces. This method provides more accurate measurement of the chamber height. 


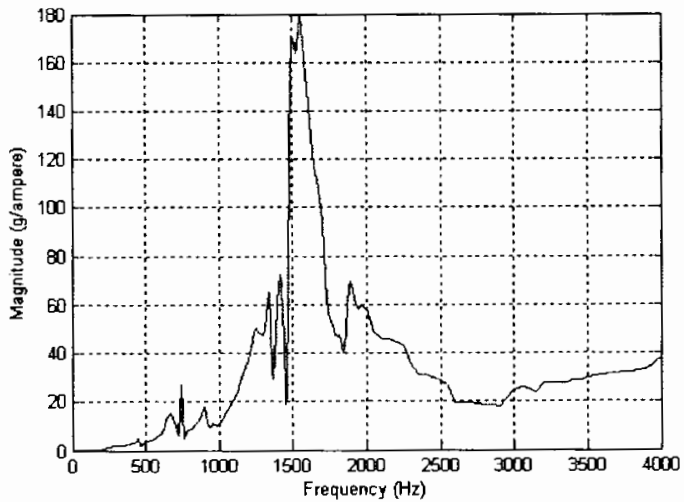

(a)

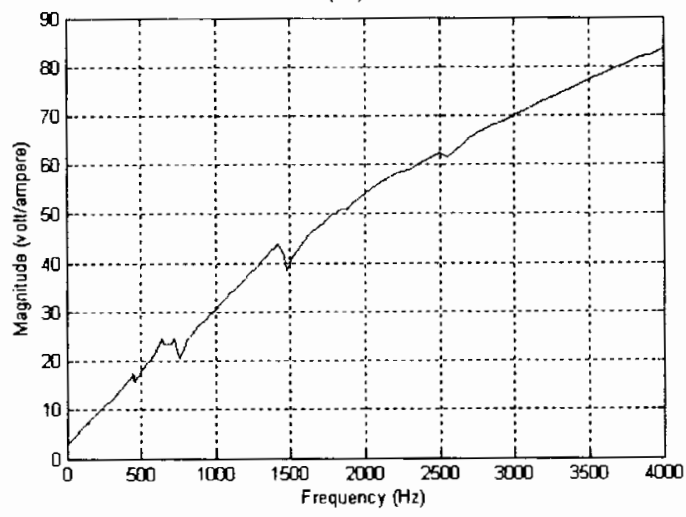

(c)

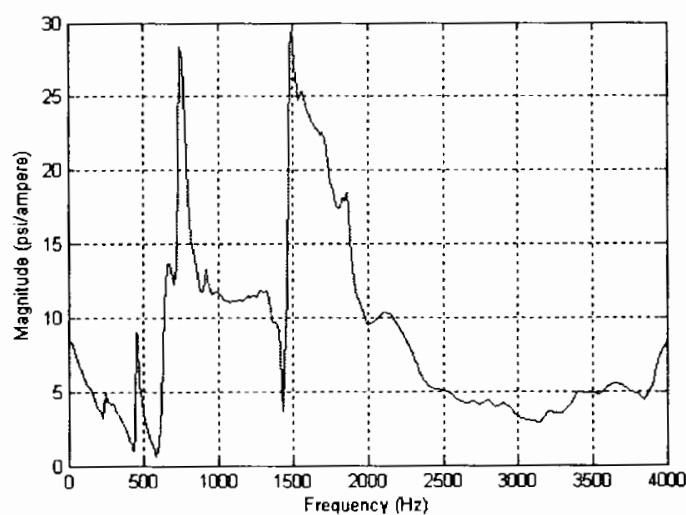

(b)

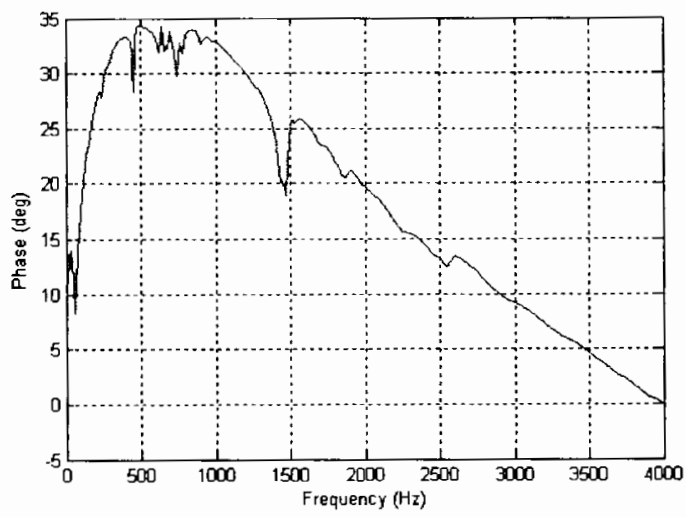

(d)

Figure C.4: Magnitude response for acceleration/input current of the piston (a), chamber pressure/input current (b) and magnitude and phase response of the impedance transfer function (c) and (d).

The next set of stepped sine measurements of the blocked chamber commences following these changes. The chamber height is set at 0.005 in which will provide a stiff fluid column. Again, the input current, voltage, piston acceleration, and chamber pressure are recorded. The results are illustrated in Fig. C.4.

Mechanical resonance of the piston occurs at $1550 \mathrm{~Hz}$ while there are two electrical resonances of the Terfenol-D transducer at $740 \mathrm{~Hz}$ and $1470 \mathrm{~Hz}$. There is also a small peak in the acceleration/input current frequency response function at $740 \mathrm{~Hz}$. 
Peaks in the chamber pressure/input current frequency response function occur at $740 \mathrm{~Hz}$ and $1490 \mathrm{~Hz}$ with magnitudes of $28.3 \mathrm{psi} / \mathrm{amp}$ and $29.4 \mathrm{psi} / \mathrm{amp}$ respectively. The pressure/input current frequency response function has a response that shows correlation between the acceleration/current and impedance frequency response functions. This is in strong contrast with the results in Fig. C.2. Above the bandwidth $(1-2000 \mathrm{~Hz})$ for the measurements using the SENSOTEC pressure sensor, there is relatively no response which is expected. Above the bandwidth $(1-1000 \mathrm{~Hz})$ for the measurements using the OMEGA pressure sensor has peaks and questionable results. Additional tests were run at $740 \mathrm{~Hz}$ and $1490 \mathrm{~Hz}$ peaks in the pressure/current frequency response function at larger applied currents in order to quantify the maximum pressures attainable by the pump. The pump created approximately $100 \mathrm{psi}$ for the $740 \mathrm{~Hz}$ peak at $1.95 \mathrm{~A}_{r m s}$ and $100 \mathrm{psi}$ for the $1490 \mathrm{~Hz}$ peak at $1.22 \mathrm{~A}_{r m s}$.

\section{C.3 Issues with Pump Design}

The first iteration of the magnetostrictive pump produced promising results and has given a direction to future pump designs. Compliance of the pump is one concern with this design. The air in the system was found to be a problem and modifications in future pump designs should address this problem. The dynamic o-ring seal may be too compliant for this application. It is not known of many high frequency applications currently utilizing dynamic o-ring for sealing purposes. For the small stroke of the Terfenol-D transducer, any loss due to this compliance will severely hurt the efficiency of the pump. This may be true for any seal that we may consider, so the seal that will optimize the pump will provide the least compliant seal. The dynamic o-ring seal may also be a source of friction loss. Proper alignment of the transducer and 
pump is essential for both the safety of the transducer and also pump performance. With such high power strokes of the Terfenol-D transducer, a large problem exists in transfer of the energy of the transducer to the fluid. Changing the rectification of the pump to high frequency check valves may address this problem and will create a unidirectional flow of the pump. 


\section{APPENDIX D}

\section{MAGNETORHEOLOGICAL VALVE DEVELOPMENT}

Concurrently with the preliminary pump design, a MR valve was developed in order to rectify magnetorheological fluid flow (see [27] for concept and background). The MR valve internal geometry was developed for optimal blocking force and a limit was set on the miniaturization of the valve. The following discussion provides an overview on the development of this valve and also on two previous valve designs.

Different MR fluid valve parameters, i.e. geometry or MR valve, were varied in order to find the optimal configuration and dimensions of the coils with miniaturization in mind. A phone conversation with Bill Bleeke, Phoenix America Inc., resulted in the understanding of a limitation in the miniaturization of the MRFV. The shaft diameter must be large enough to prevent the steel from saturating. Once the steel saturates, the magnetic induction fails to increase throughout the entire magnetic circuit. The smaller the diameter of the shaft, the quicker the steel saturates and chokes the performance of the entire magnetic circuit.

A comparison of the performance of three MR fluid valves is now given, MR fluid valves \#1 and \#2 are two existing designs including the current fixed design while MR fluid valve \#3 is a proposed new design. The best performance will yield the 
largest blocking pressures, take up the least amount of space, and have the maximum magnetic flux density directed through the MR fluid.

The geometries of focus are the two older designs with shaft diameters of 1.315 in (MRFV \#1) and 0.44 in (MRFV \#2) and flow gaps of 0.025 in and 0.030 in respectively, and the proposed model with 0.50 in shaft diameter (MRFV \#3) and 0.025 in flow gap. These three geometries will be compared by assuming the same output and flow rates. The MRFVs need to hold a $4000 \mathrm{lb}$ load. If the load is rigidly connected to a hydraulic cylinder with a 3.25 in bore and 1.0 in rod, we can define the pressure differential across the hydraulic cylinder needed to move the $4000 \mathrm{lb}$ load. The surface area of the piston head is $7.51 \mathrm{in}^{2}$. The pressure differential needed across the piston and controlled by the MRFV is defined by the equation,

$$
\Delta P_{o p}=\frac{F_{L}}{A_{L}}=\frac{4000 \mathrm{lb}}{7.51 \mathrm{in}^{2}}=533 \mathrm{psi}
$$

The pressure drop developed in a device based on pressure driven flow mode is commonly assumed to result from the sum of a viscous component $\Delta P_{\eta}$ and a field dependent induced yield stress component $\Delta P_{\tau}$. The maximum pressure that the MR valve is able to block is approximated by,

$$
\Delta P_{M R F V}=\Delta P_{\eta}+\Delta P_{\tau}=\frac{12 \eta Q L}{g^{3} W}+\frac{c \tau_{Y} L}{g}
$$

where $W=\pi d, d$ is the diameter of the valve shaft, $L$ is the total length of all magnetic pole pieces, $g$ is the gap of the flow channel, $\eta$ is the plastic viscosity of MR fluid, $Q$ is the volumetric flow rate, $\tau_{y}$ is the field dependent yield stress, and the parameter $c=3$. It is noted that $L$ is the total length of the pole pieces that yield the maximum magnetic flux density through the MR fluid and not the total length of the shaft. 
The volumetric flow rate of the Terfenol-D pump is approximated by,

$$
Q=\pi R_{p}^{2} d f
$$

Where $R_{p}$ is the radius of the input piston, $d$ is the magnitude of the deflections of the Terfenol-D rod, and $f$ is the frequency at which the Terfenol-D deflects. The equation approximates flow For a Terfenol-D rod that is 4 in long and 0.5 in diameter.

Calculations are given for when the system is both loaded and unloaded and the comparison is modeled for the smallest resulting flow rate for optimization. The peak of the magnitude response of the acceleration per input current frequency response function is located at $2700 \mathrm{~Hz}$ with a magnitude of $211 \mathrm{~g} / \mathrm{A}$. The current at this frequency is $0.340 \mathrm{~A}$.

If a sinusoidal motion is assumed, we can calculate the magnitude of the deflections of the Terfenol-D rod by,

$$
\begin{aligned}
& x(t)=d \sin (2 \pi f) \\
& x \ddot{x(t)}=-d\left(2 \pi f_{r}\right)^{2} \sin (2 \pi f)
\end{aligned}
$$

The magnitude of the acceleration can be calculated from the frequency response data and we can then solve for the magnitude of the deflections of the Terfenol-D rod,

$$
\begin{aligned}
|\ddot{x(t)}| & =d\left(2 \pi f_{r}\right)^{2} \\
d & =\frac{|x(t)|}{\left(2 \pi f_{r}\right)^{2}}
\end{aligned}
$$

Table D.1 shows $Q$ for different diameters of the drive piston.

Finite Element Analysis (FEMM) was used to quantify the Magnetic Flux Density $B$ across the MR fluid. From the B-H curve of LORD MRF-132AD, Figure D.1, the magnetic induction was found and used to find the corresponding field dependent 
yield stress through the yield stress versus magnetic induction plot, Figure D.2. The red lines in the MR charts illustrate the corresponding properties for the MR valves being investigated. The red lines near $200 \mathrm{kA} / \mathrm{m}$ correspond to MR valve \#1 while the red lines near $400 \mathrm{kA} / \mathrm{m}$ correspond to $\mathrm{MR}$ valves \#2 and \#3. The viscosity of the MR fluid is $0.09 \mathrm{~Pa} \cdot \mathrm{s}$.

The magnetic data and the geometry describing the different MRFVs are shown in Table D.2. The smallest volumetric flow rate is used for design optimization. The pressure differentials for the three different MRFV designs are approximated by the equation for $\triangle P_{M R F V}$ and the results are also shown in Table D.3. The FEMM calculations for each MRFV are displayed in the Appendix (Figs D.3-D.5).

The MRFV \#3 should be expected to substantially outperform the MRFVs \#1 and \#2. MRFV \#3 also has the theoretical ability to block a pressure differential of almost $1200 \mathrm{psi}$ which is more than double the 533 psi needed to hold the $4000 \mathrm{lb}$ load. The largest magnetic flux density was calculated for MRFV \#3, and MRFV \#3 will come in a slightly longer package than MRFV \#2.

\begin{tabular}{|c||c|}
\hline $\begin{array}{c}\text { Drive Piston Diameter } \\
{[\text { in] }}\end{array}$ & $\begin{array}{c}\text { Volume Flow Rate } \\
{\left[\mathbf{i n}^{3} / \mathbf{s}\right]}\end{array}$ \\
\hline 0.5 & 0.0509 \\
\hline 1.0 & 0.204 \\
\hline 1.5 & 0.459 \\
\hline
\end{tabular}

Table D.1: Volume flow rates for various diameters of the drive piston driven at a resonance located at $2700 \mathrm{~Hz}$. The magnitudes of deflections of the Terfenol-D rod were calculated using equation (D.7). 


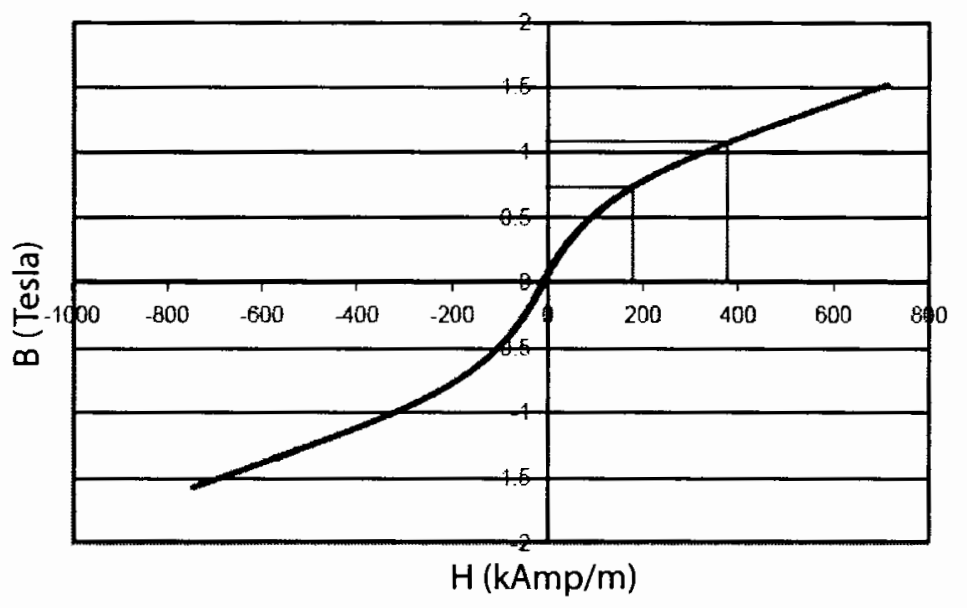

Figure D.1: Magnetic induction versus magnetic field for MRF-132AD by Lord Corporation.

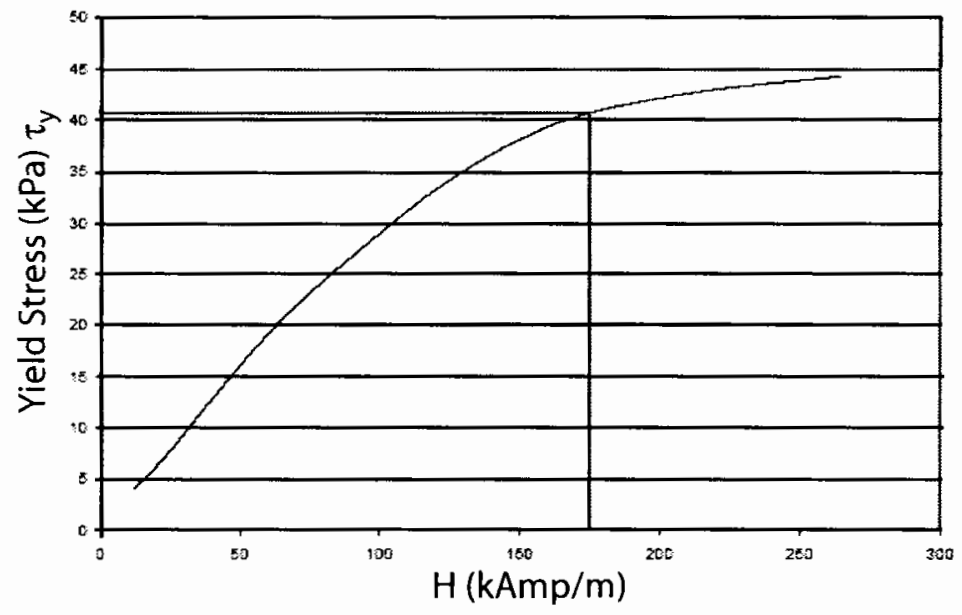

Figure D.2: Yield stress versus magnetic induction for MRF-132AD by Lord Corporation. 


\begin{tabular}{|c||c|c|c|c|c|}
\hline MRFV & $\begin{array}{c}\text { Diameter } \\
{[\mathbf{i n}]}\end{array}$ & $\begin{array}{c}\text { Length } \\
{[\mathbf{i n}]}\end{array}$ & $\begin{array}{c}\mathbf{B} \\
{[\text { Tesla }]}\end{array}$ & $\begin{array}{c}\mathbf{H} \\
{[\mathbf{k A} / \mathbf{m}]}\end{array}$ & $\begin{array}{c}\tau_{y} \\
{[\mathbf{k P a}]}\end{array}$ \\
\hline \hline 1 & 1.135 & 0.475 & 0.72 & 175 & 41 \\
\hline 2 & 0.440 & 0.125 & 1.10 & 375 & 45 \\
\hline 3 & 0.500 & 1.500 & 1.10 & 375 & 45 \\
\hline
\end{tabular}

Table D.2: Magnetic characteristics of the three different MR valve geometries calculated from FEMM and retrieved from Figures D.1-D.2.

\begin{tabular}{|c||c|c|c|}
\hline MRFV & $\begin{array}{c}\Delta P_{\eta} \\
{[\mathbf{p s i}]}\end{array}$ & $\begin{array}{c}\Delta P_{\tau} \\
{[\mathbf{p s i}]}\end{array}$ & $\begin{array}{c}\Delta P_{M R F V} \\
{[\mathbf{p s i}]}\end{array}$ \\
\hline \hline 1 & 0.06 & 338.94 & 338.99 \\
\hline 2 & 0.03 & 81.58 & 81.61 \\
\hline 3 & 0.49 & 1174.74 & 1175.23 \\
\hline
\end{tabular}

Table D.3: Results from MRFV blocked pressure differentials of three different MR valve geometries. 


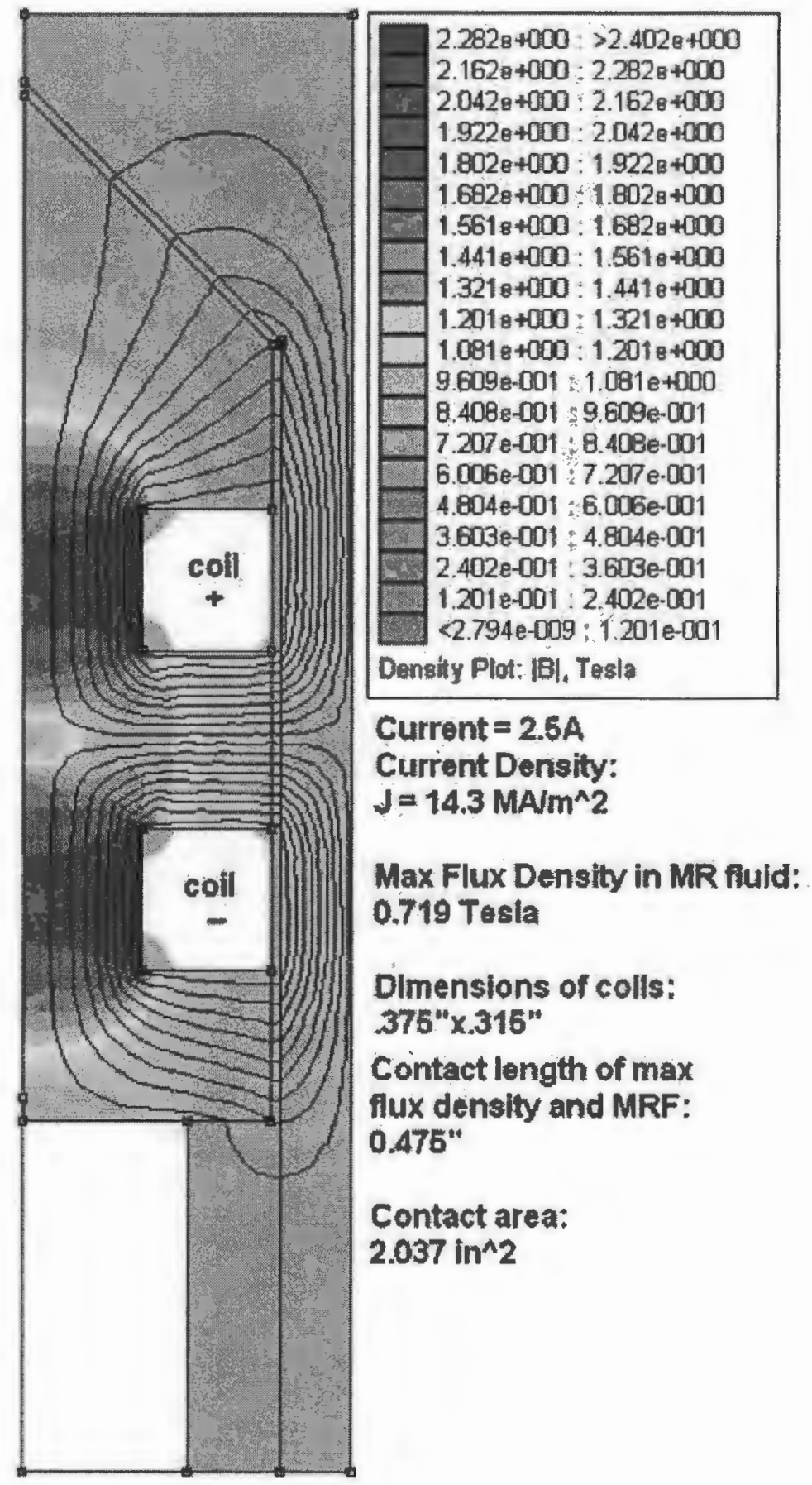

Figure D.3: FEMM - MRFV \#1, existing design. 


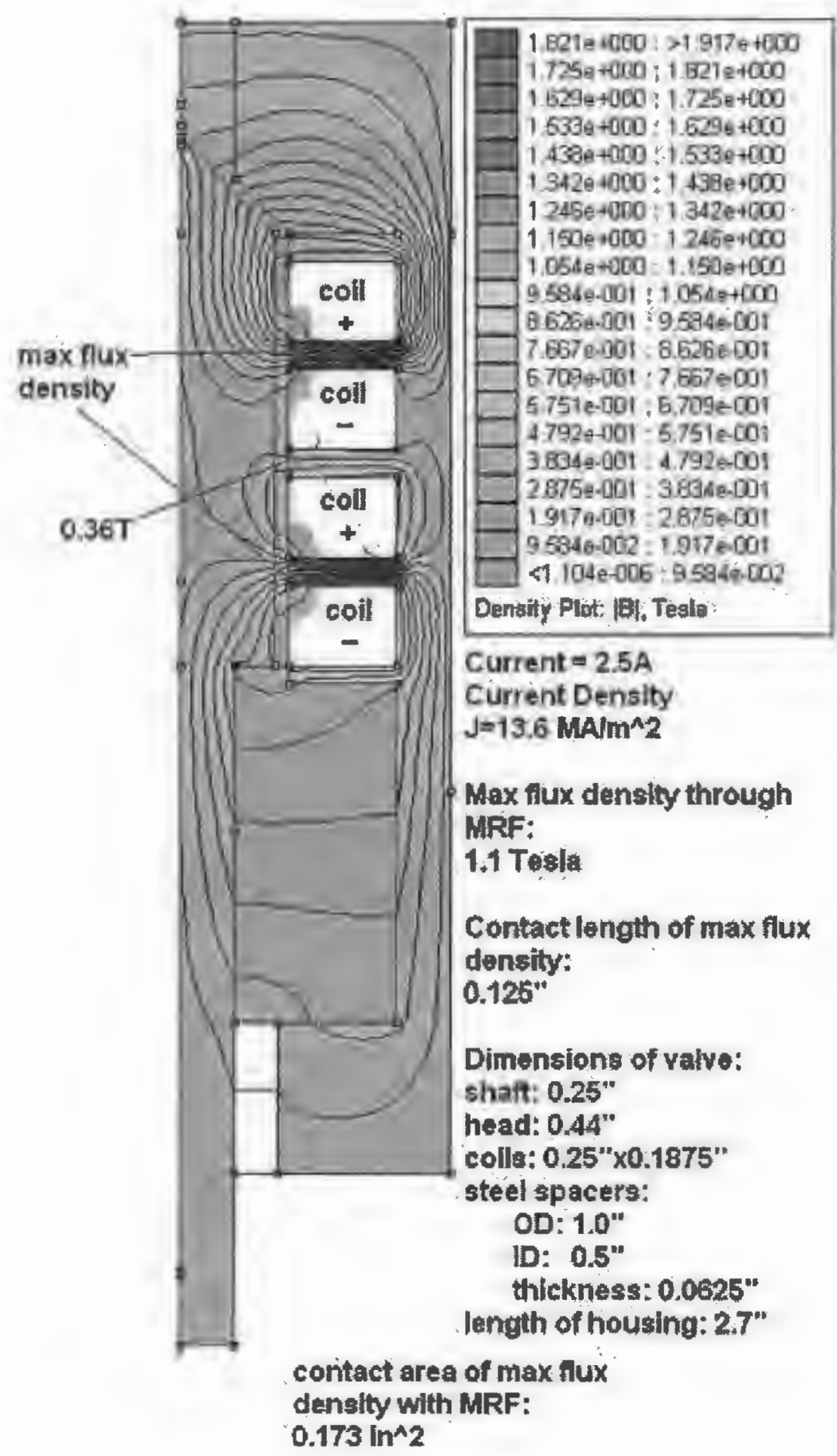

Figure D.4: FEMM - MRFV \#2, smaller existing design. 


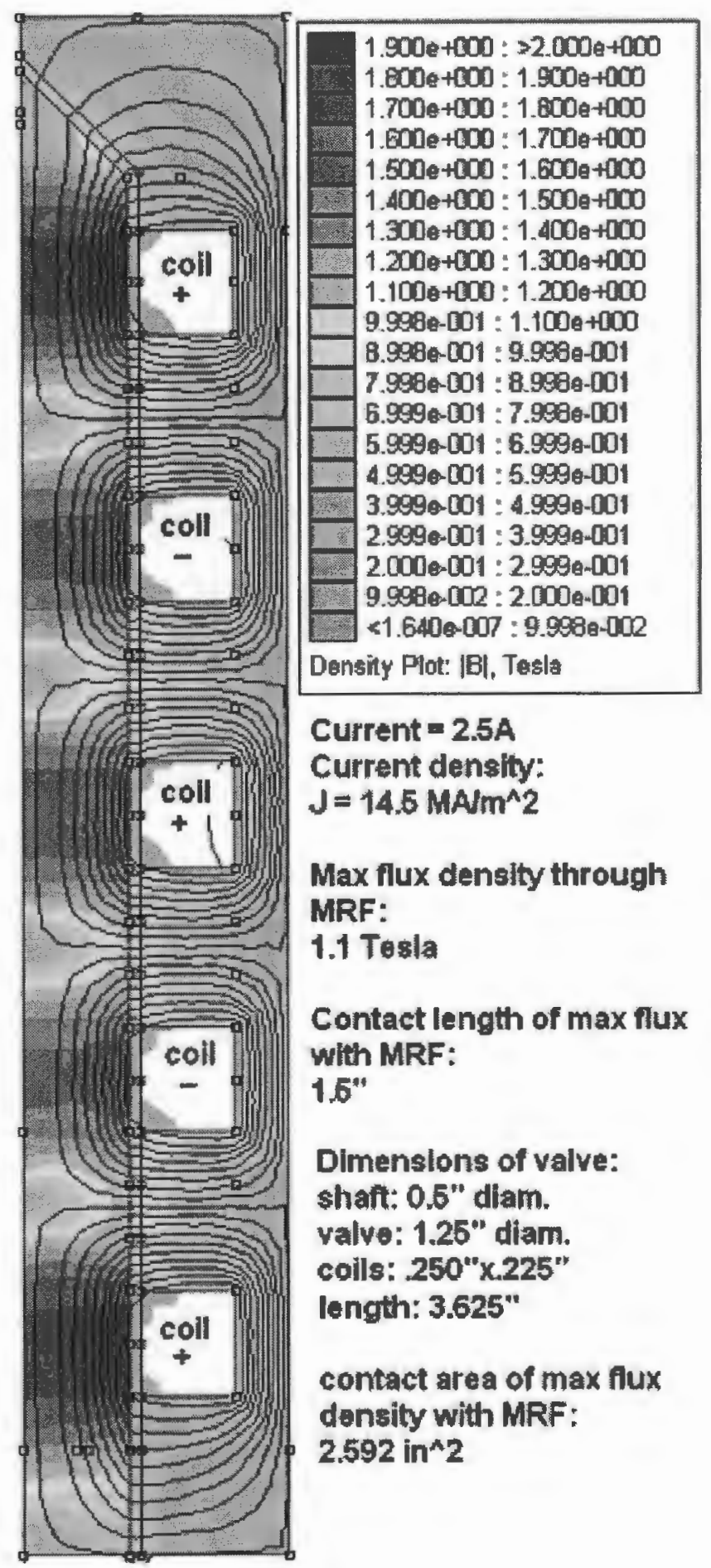

Figure D.5: FEMM - MRFV \#3, proposed design. 


\section{BIBLIOGRAPHY}

[1] T. Akuta. "Rotational type actuators with Terfenol-D rods". In Proc. 3rd Inter. Conf. New Actuators, pages 244-248, Bremen, Germany, 1992.

[2] E. H. Anderson and G. L. Bales. "Application of smart material-hydraulic actuators". In E. H. Anderson, editor, Smart Structures and Materials 2003: Industrial and Commercial Applications of Smart Structures Technologies, volume 5054 of Proceedings of SPIE, pages 73-84, 2003.

[3] D. M. Auslander, Y. Takahashi, and M. J. Rabins. "Introducing Systems and Control". McGraw-Hill, 1974.

[4] K. Bridger, J. M. Sewell, A. V. Cooke, J. L. Lutian, D. Kohlhafer, G. E. Small, and P. M. Kuhn. "High-pressure magnetostrictive pump development: a comparison of prototype and modeled performance". In E. H. Anderson, editor, Smart Structures and Materials 2004: Industrial and Commercial Applications of Smart Structures Technologies, volume 5388 of Proc. SPIE, pages 246-257, 2004.

[5] J. L. Butler, S. C. Butler, and A. L. Butler. "Hybrid magnetostrictive/piezoelectric tonpilz transducer". Journal of the Acousitical Society of America, 94:636-641, 1993.

[6] F. T. Calkins, R. C. Smith, and A. B. Flatau. "Energy-based hysteresis model for magnetostrictive transducers". IEEE Transactions on Magnetics, 36:429-439, 2000 .

[7] M. J. Dapino. "Magnetostrictive materials: Their use in smart structure applications". In J. A. Harvey, editor, Encyclopedia of Smart Materials. John Wiley and Sons, 2002.

[8] M. J. Dapino. "On magnetostrictive materials and their use in adaptive structures". Journal of Structural Engineering and Mechanics, 17:303-329, 2004.

[9] M. J. Dapino. ME794M course notes, 2005. 
[10] M. J. Dapino, A. B. Flatau, and F. T. Calkins. "Statistical analysis of terfenold material properties". Journal of Intelligent Material Systems and Structures, preprint.

[11] M. J. Dapino, R. C. Smith, and A. B. Flatau. "Structural magnetic strain model for magnetostrictive transducers". IEEE Transactions on Magnetics, 36:545-556, 2000 .

[12] P. Downey. "Hybrid PMN-PT/Terfenol-D broadband transducers in mechanical series configuration". Master's thesis, The Ohio State University, Columbus, Ohio, 2003.

[13] P. R. Downey and M. J. Dapino. "Extended frequency bandwidth and elictrical resonance tuning in hybrid terfenol-d/pmn-pt transducers in mechanical series configuration". Journal of Intelligent Material Systems and Structures, 16:757$772,2005$.

[14] C. A. Keller E. G. Chapman, S. L. Herdic and C. S. Lynch. "Development of miniaturized piezo-hydraulic pumps". In E. V. White, editor, Smart Structures and Materials 2005: Industrial and Commercial Applications of Smart Structures Technologies, volume 5762 of Proceedings of SPIE, pages 299-310, 2005.

[15] J. J. Epps and I. Chopra. "Comparitative evaluation of shape memory alloy consistutive models with test data". 38th American Institute of Aeronautics and Astronautics Structures, 1997.

[16] D. Hall. "Dynamics and vibrations of magnetostrictive transducers". PhD thesis, Iowa State University, Ames, Iowa, 1994.

[17] F. V. Hunt. "The Analysis of Transduction, and Its Historical Background". American Institute of Physics for the Acoustical Society of America, 1982.

[18] D. J. Inman. "Engineering Vibration". Prentice Hall, 2nd edition edition, 2001.

[19] C. Cadou J. Sirohi and I. Chopra. "Investigation of the dynamic characteristics of a piezohydraulic actuator". Journal of Intelligent Material Systems and Structures, 16:481-492, 2005.

[20] M. R. Jolly, J. W. Bender, and J. D. Carlson. "Properties and applications of commercial magnetorheological fluids". In L. Porter Davis, editor, Smart Structures and Materials 1998: Passive Damping and Isolation, volume 3327 of Proc. SPIE, pages 262-275, 1998.

[21] C. Keller. "Novel concepts in piezohydraulic pump designs". Master's thesis, Georgia Institute of Technology, Atlanta, Georgia, 2004. 
[22] D. G. Lee, S. Wing, and G. P. Carman. "Design of a piezoelectric-hydraulic pump with active valves". Journal of Intelligent Material Systems and Structures, 15:107-115, 2004.

[23] John R. Swenbeck R. Shea P. Jones Robert T. Ilmonen David M. Dozor S. Armstrong R. Roderick Francis E. Nimblett M. J. Gerver, James H. Goldie and R. Iovanni. "Magnetostrictive water pump". In Proceedings of SPIE Smart Structures and Materials, pages 694-705, San Diego, CA, 1998.

[24] L. D. Mauck and C. S. Lynch. "Piezoelectric hydraulic pump development". Journal of Intelligent Material Systems and Structures, 11:758-764, 2000.

[25] J. E. Meisner and J. P. Teter. "Piezoelectric/magnetostrictive resonant inchworm motor". In Proceedings of SPIE.

[26] K. Nasser and D. J. Leo. "Frequency-rectified piezohydraulic and piezopneumatic actuation". Journal of Intelligent Material Systems and Structures, 11:798-810, 2000 .

[27] D. Nosse. "Compact actuation through magnetorheological flow control and rectification of magnetostrictive vibrations". Master's thesis, The Ohio State University, Columbus, Ohio, 2005.

[28] W. S. Oates and C. S. Lynch. "Piezoelectric hydraulic pump system dynamic model". Journal of Intelligent Material Systems and Structures, 12:737-744, 2001.

[29] J. Rabinow. "The magnetic field clutch". AIEE Trans., 67:1308-1315, 1948.

[30] J-H Yoo S. John, C. Cadou and N. Wereley. "Application of cfd in the design and analysis of a piezoelectric hydraulic pump". Journal of Intelligent Material Systems and Structures, page Preprint, 2004.

[31] J. L. Shearer, B. T. Kulakowski, and J. F. Gardner. "Dynamic modeling and control of engineering systems". Prentice Hall, 2nd edition edition, 1997.

[32] J. Sirohi and I. Chopra. "Design and development of a high pumping frequency piezoelectric-hydraulic hybrid actuator". Journal of Intelligent Material Systems and Structures, 14:135-147, 2003.

[33] R. Smith. "Smart material systems: model development". Frontiers in applied mathematics, 2005.

[34] J-H. Yoo, J. Sirohi, and N. M. Wereley. "A magnetorheological piezohydraulic actuator". Journal of Intelligent Material Systems and Structures, 16:943-953, 2005. 
[35] J-H. Yoo, J. Sirohi, N. M. Wereley, and I. Chopra. "A magnetorheological hydraulic actuator driven by a piezopump". In Proc. of IMECE, 2003. 